\title{
WHEELED PROPS, GRAPH COMPLEXES AND THE MASTER EQUATION
}

\author{
M. MARKL, S. MERKULOV AND S. SHADRIN
}

\begin{abstract}
We introduce and study wheeled PROPs, an extension of the theory of PROPs which can treat traces and, in particular, solutions to the master equations which involve divergence operators. We construct a dg free wheeled PROP whose representations are in one-to-one correspondence with formal germs of SP-manifolds, key geometric objects in the theory of BatalinVilkovisky quantization. We also construct minimal wheeled resolutions of classical operads Com and Ass as rather non-obvious extensions of $\mathrm{Com}_{\infty}$ and $\mathrm{Ass}_{\infty}$, involving, e.g., a mysterious mixture of associahedra with cyclohedra. Finally, we apply the above results to a computation of cohomology of a directed version of Kontsevich's complex of ribbon graphs.
\end{abstract}

\section{Contents}

1. Introduction

2. Wheeled PROPs

2.1. Basic definitions.

2.2. Modifications and generalizations

3. Master equations

16

3.1. Differential $\mathbb{Z}_{2}$-graded Lie algebras. $\quad 16$

3.2. Differential Gerstenhaber-Batalin-Vilkovisky algebras. 17

\begin{tabular}{ll} 
3.3. & Master equation. \\
\hline
\end{tabular}

3.4. Master equations in geometry 18

4. Bar-Cobar duality for wheeled properads 22

\begin{tabular}{ll|} 
4.1. Augmentations & 22 \\
\hline
\end{tabular}

4.2. Bar and cobar $\quad 25$

5. Wheeled operads, quadratic duality and Koszulness

5.1. Wheeled operads

5.2. Quadratic duality and Koszulness 31

The first author was supported by the grant GA ČR 201/05/2117 and by the Academy of Sciences of the Czech Republic, Institutional Research Plan No. AV0Z10190503. The second author was supported by the Göran Gustafsson foundation. The third author was supported by the grants RFBR-04-02-17227-a, RFBR-05-01-02806CNRS-a, NSh-1972.2003.1, MK-5396.2006.1, NWO-RFBR-047.011.2004.026 (RFBR-05-02-89000-NWO-a), by the Göran Gustafsson foundation, and by Pierre Deligne's fund based on his 2004 Balzan prize in mathematics. 
6. Wheeled resolution of Ass and its applications 37

6.1. Wheeled completions of Ass and Ass $_{\infty}$

6.2. Directed oriented ribbon graphs $\quad 45$

6.3. Proof of Theorem C (see $\S 1)$

6.4. Proof of Corollary D (see $\S 1)$

6.5. Proof of Theorem A (see $\S 1) \quad 48$

6.6. Wheeled Massey operations 50

6.7. Cyclic characteristic class of an Ass $_{\infty}$-algebra 51

7. Wheeled Com

7.1. Resolution of wheeled Com 53

7.2. Double bar complex of Com $\quad 54$

7.3. Wheeled double bar complexes

7.4. Proof of Theorem 7.1.1

7.5. Proofs of Theorem B and Theorem 7.1.2

$\begin{array}{ll}\text { References } & 60\end{array}$

\section{INTRODUCTION}

Theory of operads and, more generally, of PROPs undergoes a rapid development in recent years [15, 19]. Originated in algebraic topology and homotopy theory, it has provided us with new powerful means to attack problems in many other areas of mathematics including deformation theory and differential geometry. Rather surprisingly, such classical systems of non-linear differential equations as Poisson equations, Nijenhuis integrability equations for an almost complex structure, or even Hochschild equations for star products on germs of smooth functions, - they all can be understood as representations of certain dg PROPs [22, 23, 24]. The common feature of all these equations is that they admit an interpretation as Maurer-Cartan equations in certain dg Lie algebras. However, in theoretical physics one often encounters not the Maurer-Cartan equations but the master equations which involve a divergence operator. Such equations can not be understood in terms of ordinary PROPs and operads as the latter have no room to encode such a basic operation in geometry and physics as trace.

In this paper we introduce and study an extension of the theory of PROPs which can treat traces and hence can be used to describe solutions to master equations. This new theory turns out to be in a sense simpler than the original one. We call it the theory of wheeled PROPs 
and operads. The motivation for the terminology is that free objects in this new category are based on directed graphs with may contain wheels, that is, directed closed paths of oriented edges. Such graph complexes as well as some methods of computing their cohomology have been studied in [24].

There is a canonical forgetful functor, PROP $\rightarrow$ PROP, from the category of wheeled PROPs to the category of PROPs which admits a left adjoint, $(-)^{\circlearrowright}:$ PROP $\rightarrow$ PROP ${ }^{\circlearrowright}$. This functor is neither exact nor full, and its study is another main purpose of our work.

This means, in particular, that a resolution, $A_{\infty}$ of, say, an operad $A$ within the category of ordinary operads may not, in general, produce via the wheeled completion $\circlearrowright$ a resolution, $\left(\mathrm{A}^{\circlearrowright}\right)_{\infty}$, of its wheeled completion, $\mathrm{A}^{\circlearrowright}$, in the category of wheeled PROPs, i.e., in general

$$
\left(\mathrm{A}^{\circlearrowright}\right)_{\infty} \neq\left(\mathrm{A}_{\infty}\right)^{\circlearrowright}
$$

This phenomenon is studied in detail for classical operads, Ass and Com, for associative and commutative algebras:

- first, we compute cohomology groups

$$
\bigoplus_{n \leq 0} H^{n}\left(\left(\mathrm{Ass}_{\infty}\right)^{\circlearrowright}, \partial\right) \quad \text { and } \quad \bigoplus_{n \leq 0} H^{n}\left(\left(\operatorname{Com}_{\infty}\right)^{\circlearrowright}, \partial\right)
$$

of the wheeled completions of their well-known minimal resolutions, $\left(\operatorname{Ass}_{\infty}, \partial\right)$ and $\left(\operatorname{Com}_{\infty}, \partial\right)$, and show that these groups are non-zero for all $n \leq 0$ (with $H^{0}\left(\left(\operatorname{Ass}_{\infty}\right)^{\circlearrowright}, \partial\right)=$ Ass $^{\circlearrowright}$ and $H^{0}\left(\left(\mathrm{Com}_{\infty}\right)^{\circlearrowright}, \partial\right)=\mathrm{Com}^{\circlearrowright}$ as expected $)$;

- second, by adding new generators we construct their wheeled minimal resolutions,

$$
\operatorname{Ass}_{\infty}^{\circlearrowright}:=\left(\mathrm{Ass}^{\circlearrowright}\right)_{\infty} \text { and } \operatorname{Com}_{\infty}^{\circlearrowright}:=\left(\mathrm{Com}^{\circlearrowright}\right)_{\infty}
$$

as rather non-trivial extensions of $\left(\text { Ass }_{\infty}\right)^{\circlearrowright}$ and, respectively, $\left(\mathrm{Com}_{\infty}\right)^{\circlearrowright}$, involving, e.g., a mysterious mixture of associahedra with cyclohedra.

More precisely, we prove the following two theorems:

Theorem A. The minimal wheeled resolution of the operad of associative algebras, Ass, is the free wheeled dg PROP, $\left(\right.$ Ass $\left._{\infty}^{\circlearrowright}, \partial\right)$, generated by

(i) planar (1,n)-corollas in degree $2-n$,

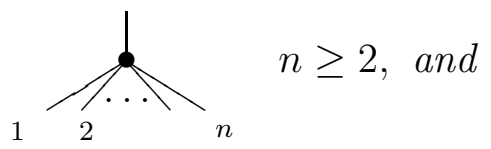

(ii) planar $(0, m+n)$-corollas in degree $-m-n$

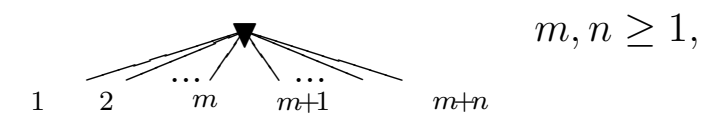


having the cyclic skew-symmetry

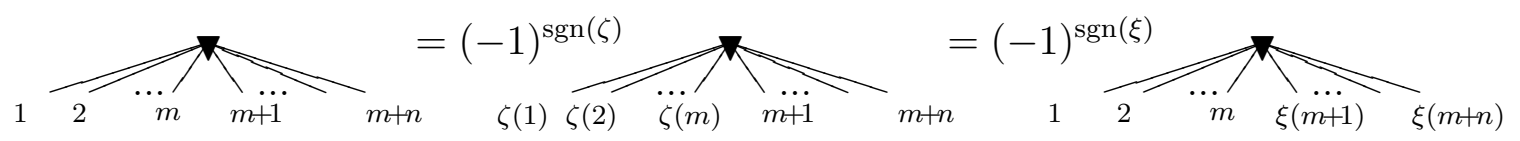

with respect to the cyclic permutations $\zeta=(12 \ldots m)$ and $\xi=((m+1)(m+2) \ldots(m+n))$. The differential is given on generators as

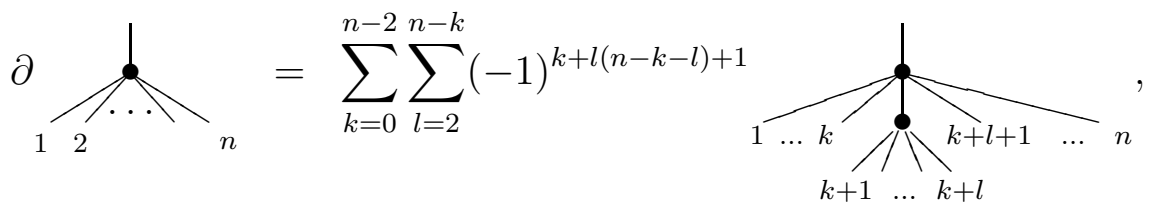

(2)

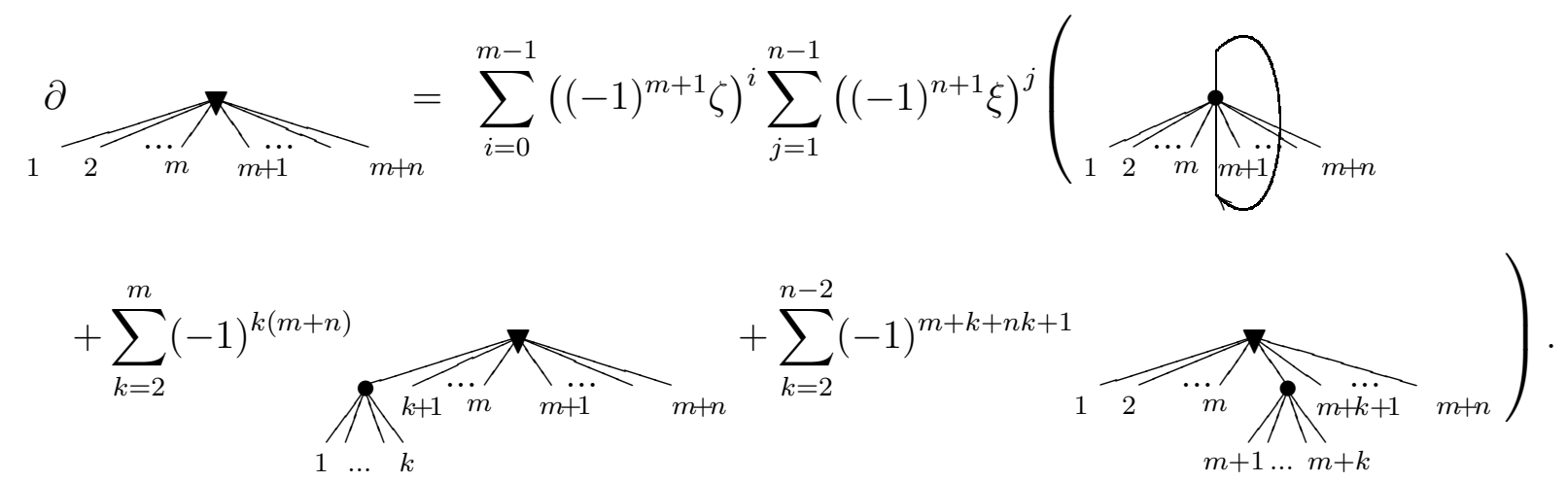

In Theorem B below, $\Sigma(k, n)$ denotes the set of all $(k, n-k)$-unshuffles,

$$
\Sigma(k, n):=\left\{\tau \in \Sigma_{n} \mid \tau(1)<\cdots<\tau(k), \tau(k+1)<\cdots<\tau(n)\right\}
$$

Theorem B. The minimal wheeled resolution of the operad of commutative associative algebras, Com, is the free wheeled $d g$ PROP, $\left(\mathrm{Com}_{\infty}^{\circlearrowright}, \partial\right)$, generated by

(i) planar $(1, n)$-corollas in degree $2-n$,

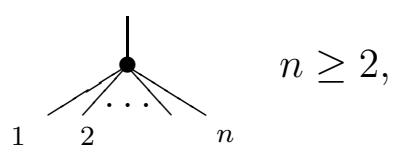

modulo the shuffle relations:

$$
\sum_{\tau \in \Sigma(k, n)}(-1)^{\operatorname{sgn}(\tau)} \overbrace{\tau(1)} \overbrace{\tau(2)}=0, \quad 1 \leq k \leq n-1, \text { and }
$$

(ii) planar $(0, n)$-corollas in degree $-n$

$$
\bigcap_{2} n \geq 2, \sigma \in \Sigma_{n}
$$

which are cyclic skew-symmetric

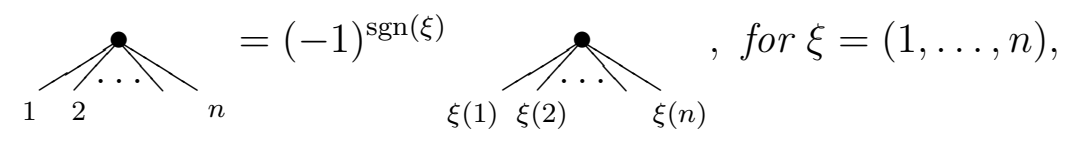


with the differential given as

(4) $\partial$

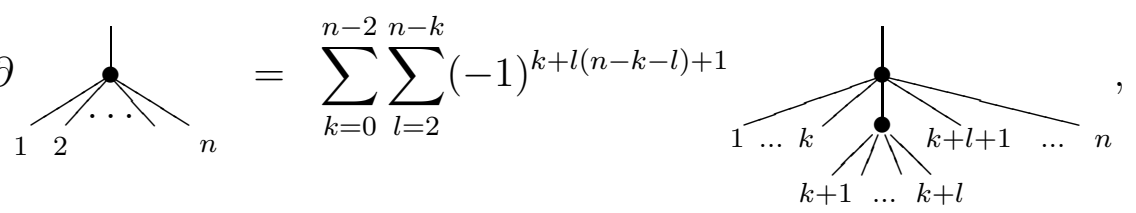

(5)
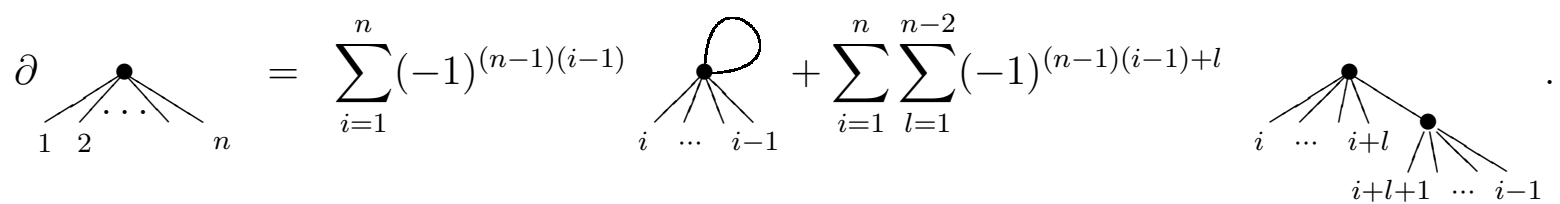

The labels in the right hand side of (5) denote cyclic permutations, so that for example $i \cdots i-1$ in the first term means $i, \ldots n, 1, \ldots, i-1$.

By contrast, the operad Lie is rigid with respect to the wheelification: it was proven in [24] that $\left(\mathrm{Lie}^{\circlearrowright}\right)_{\infty}=\left(\mathrm{Lie}_{\infty}\right)^{\circlearrowright}$.

A conceptual understanding of homological properties of a wide class of wheeled PROPs is provided by wheeled quadratic duality and wheeled Koszulness which we set up in Sections 4 and 5. This theory generalizes its "unwheeled" precursor developed in [9].

As an application of the above results we compute cohomology of a directed version of Kontsevich's complex of ribbon graphs. Let $\mathfrak{G}_{g}$ be the linear span of (not necessary connected) directed ribbon graphs of genus $g$ such that (i) each vertex has at least three attached internal edges of which at least one is incoming and at least one is outgoing, (ii) vertices in a closed path all have either precisely one incoming edge, or precisely one outgoing edge, and (iii) there are no input and output legs (i.e. every edge of the graph is internal)1. For example,

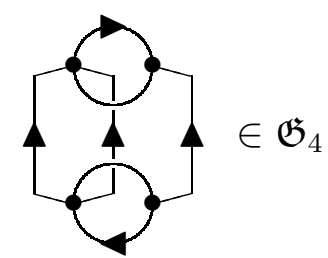

Orientation on an element $G \in \mathfrak{G}_{g}$ is, by definition, an orientation of the vector space, $\mathbb{R}^{v(G)}$, spanned by the set, $v(G)$, of vertices of $G$ (which is in fact the same as the orientation of $\mathbb{R}^{e(G)} \oplus H_{\bullet}(|G|, \mathbb{R})$, where $e(G)$ is the set of internal edges of $G$ and $H_{\bullet}(|G|, \mathbb{R})$ is the homology of $G$ viewed as a 1-dimensional $\mathrm{CW}$ complex). The space $\mathfrak{G}_{g}$ is naturally a cochain complex, $\left(\mathfrak{G}_{g}, \partial\right)$, with respect to the grading,

$$
\mathfrak{G}_{g}=\bigoplus_{n \geq 0} \mathfrak{G}_{g}^{n}
$$

by the number, $n=|v(G)|$, of vertices of its elements $G$ [11, 20].

\footnotetext{
${ }^{1}$ The space $\mathfrak{G}_{g}$ is obviously zero in the class of directed graphs without wheels. However it is highly non-trivial with wheels allowed. This simple fact provides us with one more motivation for introducing the category of wheeled PROPs.
} 
In the following theorem, $\uparrow^{k}$ denotes, for $k \geq 0$, the suspension of a graded vector space iterated $k$-times.

Theorem C. Let $\Gamma\langle E\rangle=\{\Gamma\langle E\rangle(m, n)\}_{m, n \geq 0}$ be the free (ordinary) PROP generated by the $\Sigma$-bimodule $E=\{E(m, n)\}_{m, n \geq 0}$, with

$$
E(m, n)= \begin{cases}\bigoplus_{\substack{p, q \geq 0 \\ p+q=m}} \uparrow^{m} G(p, q) \oplus \bigoplus_{\substack{p, q \geq 1 \\ p+q=m}} \uparrow^{m-1} G(p, q) & \text { for } m \geq 1, n=0, \\ \bigoplus_{\substack{p, q \geq 0 \\ p+q=n}} \uparrow^{n} G(p, q) \oplus \bigoplus_{\substack{p, q \geq 1 \\ p+q=n}} \uparrow^{n-1} G(p, q) & \text { for } n \geq 1, m=0, \\ 0 & \text { otherwise. }\end{cases}
$$

where $G(p, q):=\mathbb{R}\left[\Sigma_{p+q}\right]^{C_{p} \times C_{q}}$ is the space of coinvariants with respect to the product of cyclic subgroups $C_{p} \times C_{q} \subset \Sigma_{p+q}$ generated by permutations $(12 \ldots p)$ and $(p+1 \ldots p+q)$.

Then the graded vector space $H^{\bullet}\left(\mathfrak{G}_{g}\right)$ is isomorphic to the subspace of the component $\Gamma\langle E\rangle(0,0)$ generated by graphs with $g-1$ internal edges. In particular, $\bigoplus_{g} H^{\bullet}\left(\mathfrak{G}_{g}\right) \simeq \Gamma\langle E\rangle(0,0)$.

Corollary D. The vector space $H^{n}\left(\mathfrak{G}_{g}\right)$ is nonzero only for $n$ in the range $g-\frac{1}{2}\left(1-(-1)^{g}\right) \leq$ $n \leq 2(g-1)$.

It is worth noting another interesting phenomenon of substantial and sometimes highly nontrivial change of the set of morphisms between ordinary PROPs under their wheeled completions. For example, it was shown in [24] that deformation quantization can be understood as a morphism, DefQ $\longrightarrow$ PolyV ${ }^{\circlearrowright}$, between the dg PROP, DefQ, of star products and the wheeled completion of the dg PROP, PolyV, of polyvector fields. No such morphism (satisfying the quasiclassical limit condition), DefQ $\longrightarrow$ PolyV, exists for the original dg PROPs DefQ and PolyV within the category of ordinary PROPs.

Here is an itemized and more detailed list of main results of our paper:

In $\S 2$ we construct a triple over the category of $\Sigma$-bimodules using directed graphs with directed cycles, and then define wheeled PROPs as algebras over that triple. Modifications of this notion (such as wheeled properads and modular wheeled properads) are also given.

In $\S 3$ we construct a particular example of dg wheeled PROP whose representations are in 1-1 correspondence with the set of solutions of master equations which describe formal germs of so called SP-manifolds, key geometric objects in the theory of Batalin-Vilkovisky quantization (cf. [25]).

In $\S 4$ we introduce the notion of wheeled coproperads, define wheeled bar and cobar functors (which, rather surprisingly, turn out to be much simpler than their analogues for (co)properads) and prove Theorems 4.2.3 and 4.2.5 on bar+cobar resolutions of wheeled properads and coproperads. 
In $\S 5$ we study quadratic wheeled operads and introduce the notion of wheeled Koszulness. Theorems A and B above imply that classical wheeled operads $\mathrm{Com}^{\circlearrowright}$, Ass ${ }^{\circlearrowright}$ are wheeled Koszul (while the wheeled Koszulness of Lie ${ }^{\circlearrowright}$ was proved in [24]).

In $\S 6$ we prove Theorems A, C and D (in fact we prove a stronger Theorem 6.2.3 of which Theorem $\mathrm{C}$ is a corollary), and, as a concrete and natural example of $\mathrm{Ass}_{\infty}^{\circlearrowright}$-structure we compute explicitly new trace-type Massey operations on the homology of an arbitrary finite-dimensional dg associative algebra. We also find a new cyclic characteristic class of an arbitrary finitedimensional Ass $_{\infty}$-algebra and prove that its vanishing is a necessary and sufficient condition for extendability of the given Ass $_{\infty}$-structure to $\mathrm{Ass}_{\infty}^{\circlearrowright}$ one.

Finally, in $\S 7$ we compute cohomology of the dg properad $\left(\mathrm{Com}_{\infty}\right)^{\circlearrowright}$ and prove Theorem B.

\section{WHEELED PROPS}

2.1. Basic definitions. Let $\mathbf{k}$ denote a ground field which will always be assumed of characteristic zero. Recall that a dg (differential graded) $\mathrm{PROP}$ is a collection $\mathrm{P}=\{\mathrm{P}(m, n)\}, m, n \geq 0$, of $\operatorname{dg}\left(\Sigma_{m}, \Sigma_{n}\right)$-bimodules (left $\Sigma_{m^{-}}$right $\Sigma_{n}$-modules such that the left action commutes with the right one), together with two types of compositions, horizontal

$$
\otimes: \mathrm{P}\left(m_{1}, n_{1}\right) \otimes \cdots \otimes \mathrm{P}\left(m_{s}, n_{s}\right) \rightarrow \mathrm{P}\left(m_{1}+\cdots+m_{s}, n_{1}+\cdots+n_{s}\right),
$$

defined for all $m_{1}, \ldots, m_{s}, n_{1}, \ldots, n_{s} \geq 0$, and vertical

$$
\circ: \mathrm{P}(m, n) \otimes \mathrm{P}(n, k) \rightarrow \mathrm{P}(m, k),
$$

defined for all $m, n, k \geq 0$. These compositions are compatible with the $\mathrm{dg}$ structures. One also assumes the existence of a unit $\mathbb{1} \in \mathrm{P}(1,1)$.

PROPs should satisfy axioms which could be read off from the example of the endomorphism PROP $\mathcal{E} n d_{V}$ of a vector space $V$, with $\mathcal{E} n d_{V}(m, n)$ the space of linear maps $H o m\left(V^{\otimes n}, V^{\otimes m}\right)$ with $n$ 'inputs' and $m$ 'outputs,' $\mathbb{1} \in \mathcal{E} n d_{V}(1,1)$ the identity map, horizontal composition given by the tensor product of linear maps, and vertical composition by the ordinary composition of linear maps. For a precise definition see [14, 16].

Recall also that a $\mathrm{P}$-algebra is a PROP homomorphism $\rho: \mathrm{P} \rightarrow \mathcal{E} n d_{V}$. It is determined by a system

$$
\alpha: \mathrm{P}(m, n) \otimes V^{\otimes n} \rightarrow V^{\otimes m}, m, n, \geq 0,
$$

of linear maps satisfying appropriate axioms. P-algebras are sometimes called representations of $P$.

PROPs are devices that describe structures consisting of operations with several inputs and several outputs. Therefore various bialgebras (associative, Lie, infinitesimal) are PROPic algebras. In this section we introduce wheeled PROPs that generalize PROPs in that they describe structures whose axioms involve also "contraction of indices" or "traces." 
Before we give a precise definition of wheeled PROPs, we present the following fundamental example of this kind of structure.

2.1.1. Example. Let $V$ be a finite-dimensional vector space, with a basis $\left\{e_{s}\right\}_{1 \leq s \leq d}$. Consider a linear map $f: V^{\otimes n} \rightarrow V^{\otimes m} \in \mathcal{E} n d_{V}(m, n), m, n \geq 1$, that acts on products of basic elements by

$$
f\left(e_{\alpha_{1}} \otimes \cdots \otimes e_{\alpha_{n}}\right)=\sum M_{\alpha_{1}, \ldots, \alpha_{n}}^{\beta_{1}, \ldots, \beta_{m}} e_{\beta_{1}} \otimes \cdots \otimes e_{\beta_{m}}, 1 \leq \alpha_{s} \leq d, 1 \leq s \leq n,
$$

where $M_{\alpha_{1}, \ldots, \alpha_{n}}^{\beta_{1}, \ldots, \beta_{m}} \in \mathbf{k}$ are scalars and the sum is taken over all $1 \leq \beta_{t} \leq d, 1 \leq t \leq m$. For any pair of indices $i$ and $j, 1 \leq i \leq m, 1 \leq j \leq n$, define a multilinear map $\xi_{j}^{i}(f): V^{\otimes(n-1)} \rightarrow V^{\otimes(m-1)}$ by

$$
\xi_{j}^{i}(f)\left(e_{\mu_{1}} \otimes \cdots \otimes e_{\mu_{n-1}}\right):=\sum M_{\mu_{1}, \ldots, \mu_{j-1}, \gamma, \mu_{j}, \ldots, \mu_{n-1}}^{\nu_{1}, \ldots, \nu_{i-1}, \gamma, \nu_{i}, \ldots, \nu_{m-1}} e_{\nu_{1}} \otimes \cdots \otimes e_{\nu_{m-1}},
$$

where $1 \leq \mu_{u} \leq d, 1 \leq u \leq n-1$, and the summation runs over all $1 \leq \nu_{v}, \gamma \leq d, 1 \leq v \leq m-1$. Remarkably, the above definition of $\xi_{j}^{i}(f)$ does not depend on the basis. Formula (8) therefore defines a linear map

$$
\xi_{j}^{i}: \mathcal{E} n d_{V}(m, n) \rightarrow \mathcal{E} n d_{V}(m-1, n-1) .
$$

A "coordinate-free" definition can be given as follows. Using the duality in the category of finite-dimensional vector spaces, one can associate to any map $f: V^{\otimes n} \rightarrow V^{\otimes m}$ a map

$$
f_{j}^{i}: \operatorname{Hom}\left(V^{\otimes(n-1)}, V^{\otimes(m-1)}\right) \rightarrow \operatorname{Hom}(V, V),
$$

by singling out the $i$ th output and the $j$ th input of $f$. The composition with the ordinary trace $\operatorname{Tr}: \operatorname{Hom}(V, V) \rightarrow \mathbf{k}$ is a map $\operatorname{Tr}\left(f_{j}^{i}\right): \operatorname{Hom}\left(V^{\otimes(n-1)}, V^{\otimes(m-1)}\right) \rightarrow \mathbf{k}$ which in turn corresponds, via the duality, to the contraction $\xi_{i}^{j}(f): V^{\otimes(n-1)} \rightarrow V^{\otimes(m-1)}$ constructed above.

Wheeled PROPs are PROPs that, besides the horizontal and vertical compositions (66) and (77) admit also contractions

$$
\xi_{j}^{i}: \mathrm{P}(m, n) \rightarrow \mathrm{P}(m-1, n-1), m, n \geq 1,1 \leq i \leq m, 1 \leq j \leq n,
$$

that generalize (9) in Example 2.1.1, A precise definition of wheeled PROPs will be a modification of the unbiased definition of ordinary PROPs given in [15, Section 8] which we review below. The difference between biased and unbiased definitions is explained in [13, see also a remark in Section 3 of [15].

Recall that a $\Sigma$-bimodule is a system $E=\{E(m, n)\}_{m, n \geq 0}$ such that each $E(m, n)$ is a left $\mathbf{k}\left[\Sigma_{m}\right]$ - right $\mathbf{k}\left[\Sigma_{n}\right]$-bimodule. Let $\Sigma$-bimod denote the category of $\Sigma$-bimodules. For $E \in \Sigma$-bimod and finite sets $Y, X$ with $m$ resp. $n$ elements put

$$
E(Y, X):=\operatorname{Bij}(Y,[m]) \times_{\Sigma_{m}} E(m, n) \times_{\Sigma_{n}} \operatorname{Bij}([n], X), m, n \geq 0,
$$

where

$$
\operatorname{Bij}(T, S):=\{\vartheta: S \stackrel{\cong}{\longrightarrow} T\}
$$


is the set of bijections between finite sets $S$ and $T$, and $[m]:=\{1, \ldots, m\},[n]:=\{1, \ldots, n\}$ as usual. We are going to define an endofunctor $\Gamma$ on the category $\Sigma$-bimod that assigns to each $\Sigma$-bimodule $E$ the $\Sigma$-bimodule $\Gamma\langle E\rangle$ of $E$-decorated graphs. Unfortunately, the naive concept of graph is not refined enough for our purposes and we must recall the following more sophisticated concept taken from [12].

2.1.2. Definition. A graph $G$ is a finite set $\operatorname{Flag}(G)$ (whose elements are called flags or halfedges) together with an involution $\sigma$ and a partition $\lambda$. The vertices vert $(G)$ of a graph $G$ are the blocks of the partition $\lambda$; we assume that the number of these blocks is finite. The edges $\operatorname{Edg}(G)$ are pairs of flags forming a two-cycle of $\sigma$. The legs $\operatorname{Leg}(G)$ are the fixed points of $\sigma$.

We also denote by edge $(v)$ the flags belonging to the block $v$ or, in common speech, half-edges adjacent to the vertex $v$. We say that graphs $G_{1}$ and $G_{2}$ are isomorphic if there exists a set isomorphism $\varphi: F \operatorname{Flag}\left(G_{1}\right) \rightarrow \operatorname{Flag}\left(G_{2}\right)$ that preserves the partitions and commutes with the involutions. We may associate to a graph $G$ a finite one-dimensional cell complex $|G|$, obtained by taking one copy of $\left[0, \frac{1}{2}\right]$ for each flag, a point for each block of the partition, and imposing the following equivalence relation: The points $0 \in\left[0, \frac{1}{2}\right]$ are identified for all flags in a block of the partition $\lambda$ with the point corresponding to the block, and the points $\frac{1}{2} \in\left[0, \frac{1}{2}\right]$ are identified for pairs of flags exchanged by the involution $\sigma$.

We call $|G|$ the geometric realization of $G$. Observe that empty blocks of the partition generate isolated vertices in the geometric realization. We will usually make no distinction between the graph and its geometric realization. See [19, Section II.5.3] or [15, Section 7] for more details. A graph $G$ as in Definition 2.1.2 is a directed $(m, n)$-graph if

(i) each edge of $G$ is equipped with a direction

(ii) the set of legs of $G$ is divided into the set of inputs labeled by $\{1, \ldots, n\}$ and the set of outputs labeled by $\{1, \ldots, m\}$.

The direction of edges together with the division of legs into inputs and outputs determines at each vertex $v \in \operatorname{vert}(G)$ of a directed graph $G$ a disjoint decomposition

$$
\operatorname{edge}(v)=\operatorname{in}(v) \sqcup \operatorname{out}(v)
$$

of the set of edges adjacent to $v$ into the set $i n(v)$ of incoming edges and the set out $(v)$ of outgoing edges. The pair $(\#(\operatorname{out}(v)), \#(\operatorname{in}(v))) \in \mathbb{N} \times \mathbb{N}$ is called the biarity of $v$. By a wheel in a directed graph we mean a directed cycle of edges, see Figure 1. We usually draw directed graphs in such a way that output edges of vertices point upwards and input edges enter vertices from the bottom. We denote by $\mathrm{G}^{\uparrow}(m, n)$ the category of directed $(m, n)$-graphs without wheels and their isomorphisms. 

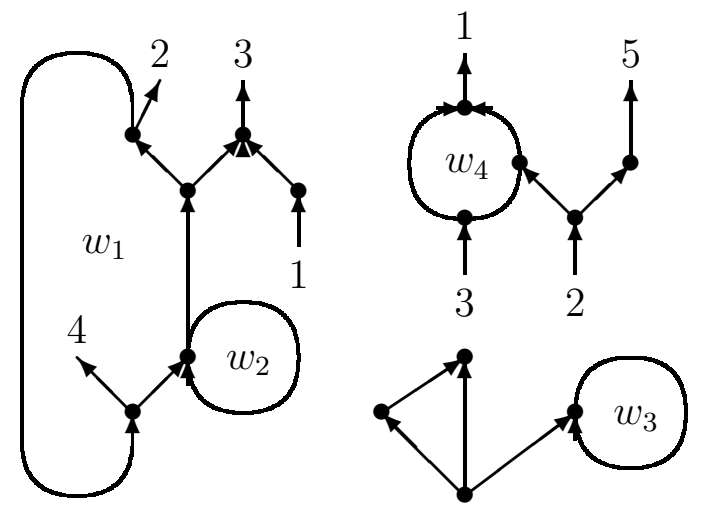

FiguRE 1. A directed $(5,3)$-graph with four independent wheels $w_{1}, w_{2}, w_{3}$ and $w_{4}$. The graph is not connected and has a component with no legs.

To incorporate the PROPeradic unit, we assume that $\mathrm{G}^{\uparrow}(m, n)$, for $m=n$, contains also the exceptional graph

$$
\uparrow \uparrow \uparrow \cdots \uparrow \in \mathrm{G}^{\uparrow}(n, n), n \geq 1,
$$

with $n$ inputs, $n$ outputs and no vertices. For a graph $G \in \mathrm{G}^{\uparrow}(m, n)$ and a $\Sigma$-bimodule $E$, let

$$
E(G):=\bigotimes_{v \in \operatorname{vert}(G)} E(\operatorname{out}(v), \operatorname{in}(v))
$$

be the linear space of all decorations of vertices of the graph $G$ by elements of $E$. Since the assignment $E \mapsto E(G)$ clearly defines a functor from the category $\mathrm{G}^{\uparrow}(m, n)$ to the category of vector spaces, it makes sense to define

$$
\Gamma\langle E\rangle(m, n):=\underset{G \in \mathrm{G}^{\uparrow}(m, n)}{\operatorname{colim}} E(G), m, n \geq 0
$$

Denote finally $\Gamma\langle E\rangle$ the $\Sigma$-bimodule $\{\Gamma\langle E\rangle(m, n)\}_{m, n \geq 0}$. Our aim now is to explain that the correspondence $E \mapsto \Gamma\langle E\rangle$ defines a triple on the category of $\Sigma$-bimodules such that PROPs are algebras over this triple.

The concept of triples and their algebras is classical, so we recall it only briefly. Let $\operatorname{End}(\mathcal{C})$ be the strict symmetric monoidal category of endofunctors on a category $\mathcal{C}$ where multiplication is the composition of functors.

2.1.3. Definition. A triple (also called a monad) $T$ on a category $\mathcal{C}$ is an associative and unital monoid $(T, \mu, v)$ in $\operatorname{End}(\mathcal{C})$. The multiplication $\mu: T T \rightarrow T$ and unit morphism $v: i d \rightarrow T$ satisfy the axioms given by commutativity of the diagrams in Figure 2.

Let us indicate how to construct transformations

$$
\mu: \Gamma \Gamma \rightarrow \Gamma \text { and } v: \mathbb{1} \rightarrow \Gamma
$$



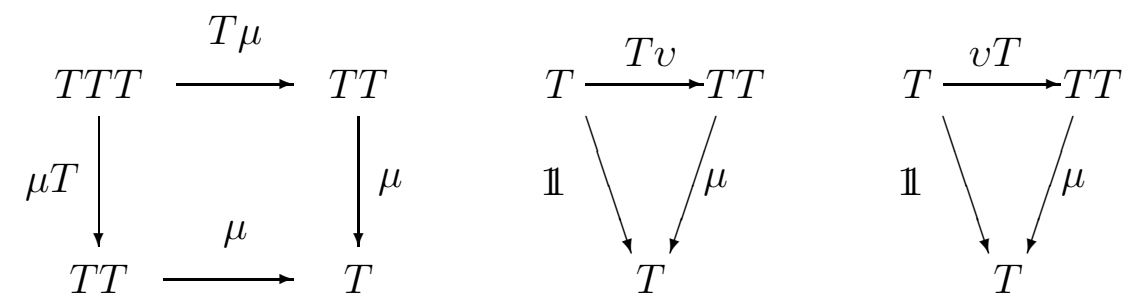

Figure 2. Associativity and unit axioms for a triple.

making $\Gamma$ a triple on the category of $\Sigma$-bimodules. Let us start with the triple multiplication $\mu$. It follows from definition (11) that, for each $\Sigma$-bimodule $E$,

$$
\Gamma\langle\Gamma\langle E\rangle\rangle(m, n):=\underset{G \in \mathrm{G}^{\uparrow}(m, n)}{\operatorname{colim}} \Gamma\langle E\rangle(G), m, n \geq 0 .
$$

The elements in the right hand side are represented by directed graphs with vertices decorated by elements of $\Gamma\langle E\rangle$, while elements of $\Gamma\langle E\rangle$ are represented by directed graphs with vertices decorated by $E$. We may therefore imagine elements of $\Gamma\langle\Gamma\langle E\rangle\rangle$ as 'bracketed' or 'nested' $E$ decorated directed graphs $G$, with nests encompassing groups of vertices of $G$ that represent one vertex decorated by an element of $\Gamma\langle E\rangle$. See also [15, Section 5] where this nesting is described and analyzed in detail. The triple multiplication $\mu_{E}: \Gamma\langle\Gamma\langle E\rangle\rangle \rightarrow \Gamma\langle E\rangle$ then simply forgets the nests. The triple unit $v_{E}: E \rightarrow \Gamma\langle E\rangle$ identifies elements of $E$ with decorated corollas:

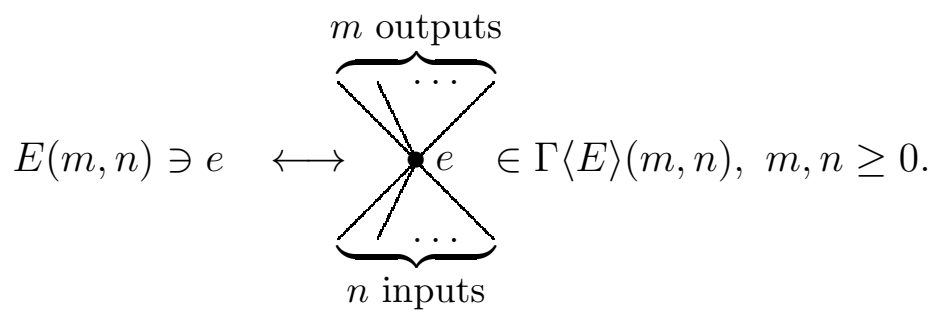

It is not difficult to verify that the above constructions indeed make $\Gamma$ a triple, compare [19, Section II.1.12] or [15, Section 5]. The last thing we need to recall is:

2.1.4. Definition. A $T$-algebra or algebra over the triple $T$ is an object $A$ of $\mathcal{C}$ together with a structure morphism $\alpha: T(A) \rightarrow A$ satisfying

$$
\alpha(T(\alpha))=\alpha\left(\mu_{A}\right) \text { and } \alpha v_{A}=\mathbb{1}_{A}
$$

see Figure 3 .

The following proposition follows from [15, Section 8], see also [27, 28].

2.1.5. Proposition. PROPs are algebras over the triple $\Gamma$. 

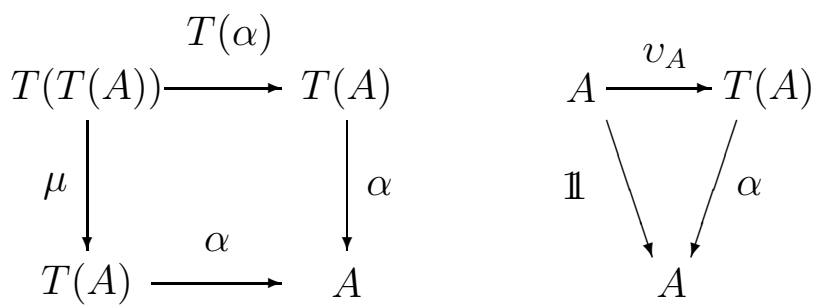

Figure $3 . \quad T$-algebra structure.

As explained in [15], the above proposition means that a PROP is given by coherent compositions along directed graphs without wheels. Therefore, a PROP is a $\Sigma$-bimodule $\mathrm{P}=$ $\{\mathrm{P}(m, n)\}_{m, n \geq 0}$ equipped with a coherent system of linear maps

$$
\alpha_{G}: \mathrm{P}(G) \rightarrow \mathrm{P}(m, n), G \in \mathrm{G}^{\uparrow}(m, n), m, n \geq 0,
$$

where $\mathrm{P}(G)$ is the space of $\mathrm{P}$-decorations of the graph $G$ introduced in (10)).

Another important observations is that the triple multiplication $\mu: \Gamma \Gamma \rightarrow \Gamma$ makes $\Gamma\langle E\rangle$ a PROP, for each $\Sigma$-bimodule $E$ - the maps (13) are the compositions

$$
\Gamma\langle E\rangle(G) \stackrel{\iota_{G}}{\longrightarrow} \Gamma\langle\Gamma\langle E\rangle\rangle \stackrel{\mu_{E}}{\longrightarrow} \Gamma\langle E\rangle,
$$

with $\iota_{G}$ the canonical map to the colimit in the right hand side of (12). It can be easily shown that the vertical composition in $\Gamma\langle E\rangle$ is given by the disjoint union of graphs, the horizontal composition by grafting the legs of graphs, and the unit is the exceptional graph $\uparrow \in \Gamma\langle E\rangle(1,1)$. The following proposition follows from general properties of algebras over triples [5].

2.1.6. Proposition. The PROP $\Gamma\langle E\rangle$ is the free PROP generated by the $\Sigma$-bimodule $E$.

Now we are finally ready to modify the above constructions and introduce wheeled PROPs, by allowing wheels in directed graphs. We start by denoting $\mathrm{G}^{\circlearrowright}(m, n), m, n \geq 0$, the category of all directed $(m, n)$-graphs and their isomorphisms. The little oriented circle in $G^{\circlearrowright}(m, n)$ indicates that wheels are allowed now. The category $\mathrm{G}^{\circlearrowright}(n, n)$ contains, for each $n \geq 0$, the exceptional graphs

$$
\uparrow \uparrow \uparrow \cdots \uparrow \circlearrowright \circlearrowright \cdots \circlearrowright \in \mathrm{G}^{\circlearrowright}(n, n), n \geq 0
$$

We denote

$$
\Gamma^{\circlearrowright}\langle E\rangle(m, n):=\underset{G \in \mathrm{G}^{\circlearrowright}(m, n)}{\operatorname{colim}} E(G), m, n \geq 0,
$$

with $E(G)$ given as in (10). We then argue as before that the above formula defines a triple $\Gamma^{\circlearrowright}$ on the category of $\Sigma$-bimodules. The central definition of this section is:

2.1.7. Definition. Wheeled PROPs are algebras over the triple $\Gamma^{\circlearrowright}: \Sigma$-bimod $\rightarrow \Sigma$-bimod. 


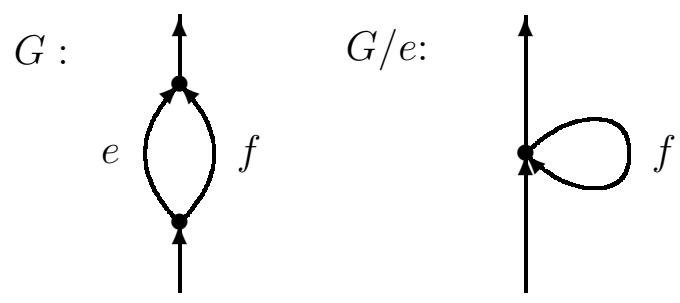

FigurE 4. A wheel created by collapsing an edge in an unwheeled graph.

The above definition can be reformulated by saying that a wheeled PROP is a $\Sigma$-bimodule $\mathrm{P}=\{\mathrm{P}(m, n)\}_{m, n \geq 0}$ with a coherent system of linear maps

$$
\alpha_{G}: \mathrm{P}(G) \rightarrow \mathrm{P}(m, n), G \in \mathrm{G}^{\circlearrowright}(m, n), m, n \geq 0,
$$

where 'coherent' means that the collection (15) assembles into a map $\alpha: \Gamma^{\circlearrowright}\langle\mathrm{P}\rangle \rightarrow \mathrm{P}$ with the properties stated in Definition 2.1.4.

As in the case of ordinary PROPs, $\Gamma^{\circlearrowright}\langle E\rangle$ carries the 'tautological' wheeled PROP structure for an arbitrary $\Sigma$-bimodule $E$. Another example of a wheeled PROP is the endomorphism PROP $\mathcal{E} n d_{V}$ of a finite-dimensional vector space $V$ discussed in Example 2.1.1,

2.1.8. Definition. Let $\mathrm{P}$ be a wheeled PROP and $V$ a finite-dimensional vector space. A Palgebra (also called a wheeled representation of $\mathrm{P}$ ) is a PROP homomorphism $\rho: \mathrm{P} \rightarrow \mathcal{E}_{n} d_{V}$, where $\mathcal{E} n d_{V}$ is the wheeled endomorphism PROP introduced in Example 2.1.1.

Surprisingly, wheeled PROPs are simpler than (ordinary) PROPs, because the category of all directed graphs is, in contrast with the category of graphs without wheels, closed under edge contractions. This means that, given a directed graph $G \in G^{\circlearrowright}(m, n)$ and an edge $e$ of $G$, the quotient $G / e$ is again a directed graph, but $G / e$ may contain wheels although $G$ does not, see Figure 4. The consequence of this observation is that the compositions (15) in a wheeled PROP are generated by edge contractions and disjoint unions of graphs.

Therefore a biased definition of wheeled PROPs can be given in terms of the horizontal compositions (6) that correspond to disjoint unions of graphs, and the contractions

$$
\xi_{j}^{i}: \mathrm{P}(m, n) \rightarrow \mathrm{P}(m-1, n-1)
$$

defined for $1 \leq i \leq m, 1 \leq j \leq n$, that correspond to the graph in Figure 5. The 'dioperadic' (see [6] for the terminology) compositions

$$
\circ_{j}^{i}: \mathrm{P}\left(m_{1}, n_{1}\right) \otimes \mathrm{P}\left(m_{2}, n_{2}\right) \rightarrow \mathrm{P}\left(m_{1}+m_{2}-1, n_{1}+n_{2}-1\right),
$$

where $m_{1}, n_{2} \geq 0,1 \leq i \leq n_{1}, 1 \leq j \leq m_{2}$, corresponding to the contraction of an edge joining two different vertices, can be expressed in terms of the above two operations as

$$
p \circ_{j}^{i} q=\xi_{i}^{m_{1}+j}(p \otimes q), p \in \mathrm{P}\left(m_{1}, n_{1}\right), q \in \mathrm{P}\left(m_{2}, n_{2}\right),
$$




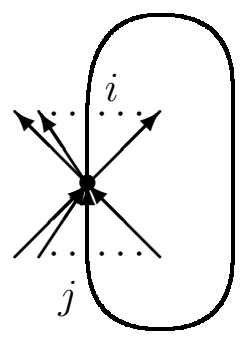

FiguRE 5. The graph generating contractions (16). Its vertex has biarity $(m, n)$.

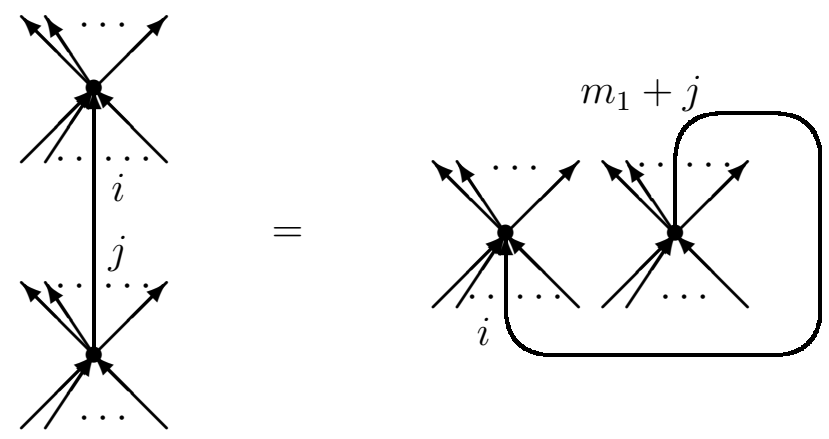

Figure 6. A dioperadic composition as a horizontal compositions followed by a contraction. The upper vertex of the left graph has biarity $\left(m_{1}, n_{1}\right)$, the bottom vertex biarity $\left(m_{2}, n_{2}\right)$.

see Figure 6. We are, however, not going to write axioms that these operations should fulfill here.

The obvious forgetful functor $\square:$ PROP $^{\circlearrowright} \rightarrow$ PROP from the category of wheeled PROPs to the category of PROPs is induced by the natural inclusions $\mathrm{G}^{\uparrow}(m, n) \hookrightarrow \mathrm{G}^{\circlearrowright}(m, n), m, n \geq 0$. It is easy to show that $\square$ has a left adjoint $(-)^{\circlearrowright}:$ PROP $\rightarrow$ PROP

2.1.9. Definition. Given a PROP P, we call the wheeled PROP P $\mathrm{P}^{\circlearrowright}$ the wheeled completion of $\mathrm{P}$.

2.1.10. Proposition. For any PROP P there is a one-to-one correspondence between finite dimensional representations of $\mathrm{P}$ in the category $\mathrm{PROP}$ and finite dimensional representations of $\mathrm{P} \circlearrowright$ in the category of PROP ${ }^{\circlearrowright}$.

2.1.11. Definition. For a graded vector space $V$, let $\uparrow V$ be the suspension of $V$ defined by $(\uparrow V)_{i}:=V_{i-1}$, and let $\downarrow V$ be the desuspension of $V$ defined by $(\uparrow V)_{i}:=V_{i+1}$.

The suspension wheeled properad, $\mathbf{S}$, is the endomorphism wheeled prop(erad) of $\uparrow \mathbf{k}$ and the desuspension wheeled properad, $\mathbf{S}^{-1}$, is the endomorphism wheeled prop(erad) of $\downarrow \mathbf{k}$. Thus $\mathbf{S}$ is the one dimensional $\Sigma$-bimodule $\left\{\uparrow^{m-n} \operatorname{sgn}_{m} \otimes \operatorname{sgn}_{n}\right\}$ and $S^{-1}$ is the one dimensional $\Sigma$-bimodule $\left\{\downarrow^{m-n} \operatorname{sgn}_{m} \otimes \operatorname{sgn}_{n}\right\}$. Tensoring with $S\left(\right.$ or with $S^{-1}$ ) defines an endomorphism functor in the category of wheeled prop(erad)s. 
2.2. Modifications and generalizations. In [27, 28], B. Vallette introduced properads as a suitable subcategory of the category of (ordinary) PROPs on which (co)bar constructions and quadratic duality could be defined. We will need, in Section 4, a wheeled version of this notion. Recall that Vallette's properads are algebras over a subtriple $\mathrm{F}$ of the free PROP triple $\Gamma$ given as the colimit over connected graphs, that is, for a $\Sigma$-bimodule $E, \mathrm{~F}\langle\mathrm{E}\rangle$ is defined by

$$
\mathrm{F}\langle\mathrm{E}\rangle(m, n):=\underset{G \in \mathrm{G}_{c}^{\uparrow}(m, n)}{\operatorname{colim}} E(G), m, n \geq 0,
$$

where $\mathrm{G}_{c}^{\uparrow}(m, n)$ is the full subcategory of $\mathrm{G}^{\uparrow}(m, n)$ consisting of connected graphs. Similarly, there is a subtriple $\mathrm{F}^{\circlearrowright}$ of the triple $\Gamma^{\circlearrowright}$ defined by

$$
\mathrm{F}^{\circlearrowright}\langle E\rangle(m, n):=\underset{G \in \mathrm{G}_{c}^{\circlearrowright}(m, n)}{\operatorname{colim}} E(G), m, n \geq 0,
$$

where $\mathrm{G}_{c}^{\circlearrowright}(m, n)$ is the full subcategory of $\mathrm{G}^{\circlearrowright}(m, n)$ of connected graphs. Observe that there are precisely two connected exceptional graphs,

$$
\uparrow \in \mathrm{G}_{c}^{\circlearrowright}(1,1) \text { and } \circlearrowright \in \mathrm{G}_{c}^{\circlearrowright}(0,0) \text {. }
$$

2.2.1. Definition. A wheeled properad is an algebra over the triple $\mathrm{F}^{\circlearrowright}$ introduced above.

It is clear that, for each $\Sigma$-bimodule, $\mathrm{F}^{\circlearrowright}\langle E\rangle$ is a wheeled properad, with the structure map given by the triple multiplication $\mu_{E}: \mathrm{F}^{\circlearrowright}\left\langle\mathrm{F}^{\circlearrowright}\langle E\rangle\right\rangle \rightarrow \mathrm{F}^{\circlearrowright}\langle E\rangle$. It is the free wheeled properad generated by the $\Sigma$-bimodule $E$.

Observe that each wheeled properad generates a wheeled PROP, but not all wheeled PROPs are generated by wheeled properads. Each properad $\mathrm{P}$ has its wheeled properadic completion $\mathrm{P}_{c}^{\circlearrowright}$ given by an obvious modification of Definition 2.1.9.

2.2.2. Example. Let $\mathfrak{k}$ be the trivial properad (the initial object of the category properads) defined by

$$
\mathfrak{k}(m, n):= \begin{cases}\mathbf{k}, & \text { for }(m, n)=(1,1), \text { and } \\ 0, & \text { otherwise. }\end{cases}
$$

Its wheeled properadic completion $\mathfrak{k}_{c}^{\circlearrowright}$ is the initial object of the category of wheeled properads. It satisfies

$$
\mathfrak{k}_{c}^{\circlearrowright}(m, n):= \begin{cases}\mathbf{k}, & \text { for }(m, n)=(1,1) \text { or }(0,0), \text { and } \\ 0, & \text { otherwise. }\end{cases}
$$

The component $\mathfrak{k}_{c}^{\circlearrowright}(0,0)$ is spanned by the contraction of the unit $1 \in \mathfrak{k}_{c}^{\circlearrowright}(1,1)$. Of course, $\mathfrak{k}_{c}^{\circlearrowright}$ equals $\mathrm{F}^{\circlearrowright}\langle 0\rangle$, the free wheeled properad generated by the trivial $\Sigma$-bimodule.

We close this section by mentioning an important modification of wheeled properads whose nature resembles modular operads introduced in [8]. Let us recall some necessary definitions. 
A labeled graph is a connected graph $G$ together with a map $g$ from $\operatorname{vert}(G)$ to the set $\{0,1,2, \ldots\}$. The genus $g(G)$ of a labeled graph $G$ is defined by

$$
g(G):=\operatorname{dim} H_{1}(G)+\sum_{v \in \operatorname{vert}(G)} g(v) .
$$

Let us denote by $\mathrm{G}_{c}^{\circlearrowright}(g ; m, n)$ the category of labeled directed $(m, n)$-graphs and their isomorphisms.

By a modular $\Sigma$-bimodule we mean a system $E=\{E(g ; m, n)\}_{g, m, n \geq 0}$ such that each $E(g ; m, n)$ is a left $\mathbf{k}\left[\Sigma_{m}\right]$ - right $\mathbf{k}\left[\Sigma_{n}\right]$-bimodule. For a modular $\Sigma$-bimodule $E$ and $G \in \mathrm{G}_{c}^{\circlearrowright}(g ; m, n)$, let

$$
E(G):=\bigotimes_{v \in \operatorname{vert}(G)} E(g(v) ; \operatorname{out}(v), \operatorname{in}(v))
$$

Define finally

$$
\mathrm{MF}^{\circlearrowright}\langle E\rangle(g ; m, n):=\underset{G \in \mathrm{G}_{c}^{\circlearrowright}(g ; m, n)}{\operatorname{colim}} E(G), g, m, n \geq 0 .
$$

As before, $\mathrm{MF}^{\circlearrowright}$ is a triple in the category of modular $\Sigma$-bicollections.

2.2.3. Definition. Modular wheeled properads are algebras over the triple $\mathrm{MF}^{\circlearrowright}$.

Loosely speaking, modular wheeled properads are wheeled properads equipped with a genus grading such that the dioperadic compositions (17) preserve the genus and the contractions (16) increase the genus by one. One may also say that modular wheeled properads are directed (unstable) modular operads.

2.2.4. Example. For each $\Sigma$-bimodule $E$, the free wheeled properad $\mathrm{F}^{\circlearrowright}\langle E\rangle$ is modular, with the genus grading induced by the genus of underlying graphs. Obviously, each wheeled properad $\mathrm{P}$ of the form $\mathrm{P}=\mathrm{F}^{\circlearrowright}\langle E\rangle / I$, with the ideal $I$ generated by elements supported by genus zero graphs, has the induced modular structure. This means that the wheeled properadic completion of a dioperad [6] is a modular wheeled properad.

2.2.5. Example. If one allows the genus to be an arbitrary integer, then each wheeled properad $\mathrm{P}$ can be turned into a modular one by assigning $\mathrm{P}(m, n)$ the genus $1-m$.

\section{MASTER EQUATIONS}

3.1. Differential $\mathbb{Z}_{2}$-graded Lie algebras. In the context of Batalin-Vilkovisky formalism in quantum field theory it is more suitable to work with the odd version of the usual notion of differential Lie superalgebra. By definition, this is a $\mathbb{Z}_{2}$-graded vector space, $\mathfrak{g}=\mathfrak{g}_{0} \oplus \mathfrak{g}_{\tilde{1}}$, equipped with two odd linear maps

$$
d: \mathfrak{g} \rightarrow \mathfrak{g}, \quad \text { and } \quad[\bullet]: \mathfrak{g} \otimes \mathfrak{g} \rightarrow \mathfrak{g},
$$


such that $d^{2}=0,[a \bullet b]=-(-1)^{(\tilde{a}+1)(\tilde{b}+1)}[b \bullet a]$,

$$
d[a \bullet b]=[d a \bullet b]+(-1)^{(\tilde{a}+1)}[a \bullet d b],
$$

and

$$
[a \bullet[b \bullet c]]=[[a \bullet b] \bullet c]+(-1)^{(\tilde{a}+1)(\tilde{b}+1)}[b \bullet[a \bullet c],
$$

for all $a, b, c \in \mathfrak{g}_{\tilde{0}} \cup \mathfrak{g}_{\tilde{1}}$.

In many important examples, the $\mathbb{Z}_{2}$-grading in $\mathfrak{g}$ comes from an underlying $\mathbb{Z}$-grading, i.e. $\mathfrak{g}=\oplus_{i \in \mathbb{Z}} \mathfrak{g}^{i}, \mathfrak{g}_{\tilde{0}}=\oplus_{i \text { even }} \mathfrak{g}^{i}, \mathfrak{g}_{\tilde{1}}=\oplus_{i \text { odd }} \mathfrak{g}^{i}$, and the basic operations satisfy $d \mathfrak{g}^{i} \subset \mathfrak{g}^{i+1},\left[\mathfrak{g}^{i} \bullet \mathfrak{g}^{j}\right] \subset$ $\mathfrak{g}^{i+j-1}$.

Clearly, the parity change functor transforms this structure into the ordinary structure of differential Lie superalgebra on the vector superspace $\Pi \mathfrak{g}$.

3.2. Differential Gerstenhaber-Batalin-Vilkovisky algebras. Such an algebra is a quadruple $(\mathfrak{g}, \circ, d, \Delta)$, where $(\mathfrak{g}, \circ)$ is a unital supercommutative algebra over a field $k$, and $(d, \Delta)$ is a pair of supercommuting odd derivations of $(\mathfrak{g}, \circ)$ of orders 1 and, respectively, 2 which satisfy $d^{2}=\Delta^{2}=0$.

More explicitly, a dGBV algebra is a differential supercommutative algebra with unit, $(\mathfrak{g}, \circ, d)$, equipped an odd linear map $\Delta: \mathfrak{g} \rightarrow \mathfrak{g}$ satisfying

(i) $\Delta^{2}=0$,

(ii) $d \Delta+\Delta d=0$, and

(iii) for any $a, b, c \in \mathfrak{g}$,

$$
\begin{aligned}
\Delta(a \circ b \circ c)= & \Delta(a \circ b) \circ c+(-1)^{\tilde{b}(\tilde{a}+1)} b \circ \Delta(a \circ c)+(-1)^{\tilde{a}} a \circ \Delta(b \circ c) \\
& -\Delta(a) \circ b \circ c-(-1)^{\tilde{a}} a \circ \Delta(b) \circ c-(-1)^{(\tilde{a}+\tilde{b})} a \circ b \circ \Delta(c) .
\end{aligned}
$$

Note that $\Delta(1)=0$.

It is not hard to check using identity (iii) that the linear map

$$
\begin{aligned}
{[\bullet]: \mathfrak{g} \otimes \mathfrak{g} } & \longrightarrow \mathfrak{g} \\
a \otimes b & \longrightarrow[a \bullet b]:=(-1)^{\tilde{a}} \Delta(a \circ b)-(-1)^{\tilde{a}} \Delta(a) \circ b-a \circ \Delta(b)
\end{aligned}
$$

makes $\mathfrak{g}$ into an odd Lie superalgebra.

Moreover, both triples $(\mathfrak{g},[\bullet], d)$ and $(\mathfrak{g},[\bullet], \Delta)$ are odd differential Lie superalgebras, and the following odd Poisson identity,

$$
[a \bullet(b \circ c)]=[a \bullet b] \circ c+(-1)^{\tilde{a}(\tilde{b}+1)} b \circ[a \bullet b],
$$

holds for any $a, b, c \in \mathfrak{g}$. 
3.3. Master equation. An even element $S$ in a dGBV algebra $\mathfrak{g}$ is called a master function if it satisfies the master equation

$$
d S+\Delta S+\frac{1}{2}[S \bullet S]=0
$$

Assume that $\mathfrak{g}$ is such that the formal power series,

$$
e^{S}=1+S+\frac{1}{2 !} S \circ S+\frac{1}{3 !} S \circ S \circ S+\ldots,
$$

makes sense, i.e. gives a well-defined element of $\mathfrak{g}$ (often this is achieved by introducing a formal parameter $\hbar$ and working in $\mathfrak{g}[[\hbar]])$. One of the central observation in the theory of master equations is the following

3.3.1. Lemma. $S$ is a master function if and only if

$$
(d+\Delta) e^{S}=0 .
$$

Proof. One checks that $(d+\Delta) e^{S}=\left(d S+\Delta S+\frac{1}{2}[S \bullet S]\right) \circ e^{S}$.

3.4. Master equations in geometry. Let $M$ be a smooth $n$-dimensional manifold with the tangent sheaf denoted by $T_{M}$ and the sheaf of differential forms denoted by $\Omega_{M}^{\bullet}=\bigoplus_{i=0}^{n} \Omega_{M}^{i}$. It is well-known that the sheaf of polyvector fields,

$$
\wedge^{\bullet} T_{M}=\bigoplus_{i=0}^{n} \wedge^{i} T_{M}
$$

is a sheaf of supercommutative algebras with respect to the wedge product, $\circ=\wedge$, and also a sheaf of odd Lie superalgebras with respect to the Schouten bracket,

$$
[\bullet]_{\text {Schouten }}: \wedge^{i} T_{M} \otimes \wedge^{j} T_{M} \longrightarrow \wedge^{i+j-1} T_{M} .
$$

Moreover, the odd Poisson identity,

$$
[a \bullet(b \wedge c)]_{\text {Schouten }}=[a \bullet b]_{\text {Schouten }} \wedge c+(-1)^{\tilde{a}(\tilde{b}+1)} b \wedge[a \bullet b]_{\text {Schouten }},
$$

holds for any $a, b, c \in \wedge^{\bullet} T_{M}$.

Assume now that $M$ is equipped with a volume form $\nu$, that is, with a nowhere vanishing section of $\Omega_{M}^{n}$. In particular, the associated section $\nu^{-1}$ of $\wedge^{n} T_{M}$ is well defined.

Define a differential operator $\Delta: \wedge^{\bullet} T_{M} \rightarrow \wedge^{\bullet} T_{M}$ as the composition

$$
\Delta: \wedge^{i} T_{M} \stackrel{\lrcorner \nu}{\longrightarrow} \Omega_{M}^{n-i} \stackrel{d}{\longrightarrow} \Omega_{M}^{n-i+1} \stackrel{\lrcorner \nu^{-1}}{\longrightarrow} \wedge^{i-1} T_{M}
$$

where $d$ stands for de Rham differential and $\lrcorner$ for the natural contraction of dual tensors. It is well-known (and easy to check, see a local coordinate description below) that the data $\left(\wedge^{\bullet} T_{M}, d=\right.$ $0, \Delta)$ is dGBV algebra with the associated odd Lie algebra structure being exactly the Schouten structure. The associated master equation has the form

$$
\Delta S+\frac{1}{2}[S \bullet S]_{\text {Schouten }}=0 .
$$


Sections of the bundle $\wedge^{\bullet} T_{M}$ can be understood as functions on the supermanifold $\Pi \Omega_{M}^{1}$, the total space of the cotangent bundle with the parity of fiber coordinates changed. If $\left\{x^{\alpha}\right\}_{1 \leq \alpha \leq n}$ are local coordinates on $M$, then the functions $\left\{x^{\alpha}, \psi_{\alpha}:=\Pi \partial / \partial x^{\alpha}\right\}_{1 \leq \alpha \leq n}$ form a local coordinate system on $\Pi \Omega_{M}^{1}$. The volume form $\nu$ gets the coordinate representation $\nu=f d x^{1} \wedge \ldots \wedge d x^{n}$ for some non-vanishing function $f$, while the divergence operator $\Delta$ gets explicitly represented as

$$
\Delta=\sum_{\alpha=1}^{n} \frac{\partial^{2}}{\partial x^{\alpha} \partial \psi_{\alpha}}+\frac{\partial \ln f}{\partial x^{\alpha}} \frac{\partial}{\partial \psi_{\alpha}} .
$$

Hence one has are

$$
[f \bullet g]_{\text {Schouten }}=\sum_{\alpha=1}^{n}\left(\frac{\partial f}{\partial x^{\alpha}} \frac{\partial g}{\partial \psi_{\alpha}}+(-1)^{\tilde{g} \tilde{f}} \frac{\partial f}{\partial x^{\alpha}} \frac{\partial g}{\partial \psi_{\alpha}}\right) .
$$

for arbitrary $f, g \in \wedge^{\bullet} T_{M}$.

3.4.1. Calabi-Yau manifolds. There is a variant of the above master equation for CY manifolds with $\nu$ being the holomorphic volume form. If one defines $\Delta_{\partial}$ on holomorphic vector fields, $\wedge^{\bullet} \mathcal{T}_{M}$, precisely as above with the full de Rham differential $d=\partial+\bar{\partial}$ replaced by its $(1,0)$-part $\partial$, then the sheaf $\wedge^{\bullet} \mathcal{T}_{M} \otimes \Omega_{M}^{0, \bullet}$ is a dGBV algebra with differential $d:=\operatorname{Id} \otimes \bar{\partial}$ and the 2 nd order derivation $\Delta:=\Delta_{\partial} \otimes \mathrm{Id}$. The associated master equation

$$
d S+\hbar \Delta S+\frac{1}{2}[S \bullet S]=0, \quad S \in \wedge^{\bullet} \mathcal{T}_{M} \otimes \Omega_{M}^{0, \bullet}[[\hbar]]
$$

plays a key role in the Barannikov-Kontsevich approach [1, 3] to the $B$-model side of the Mirror Symmetry. It solutions describe extended deformations of the complex structure on a Calabi-Yau manifold.

3.4.2. Master equation on supermanifolds and complexes. In theoretical physics, one is more interested in a version of the construction in $\$ 3.4$ when the underlying space $M$ is a supermanifold rather than a manifold. In both cases the supermanifold $\mathcal{M}:=\Pi \Omega^{1} M$ has an odd symplectic structure $\omega \in \Omega^{2} \mathcal{M}$, but the notions of volume forms are different - in the supermanifold case it should be understood as a section of the Berezinian bundle, rather than a differential form. Given such a section, $\nu \in \operatorname{Ber}(\mathcal{M})$, one obtains, for an arbitrary vector field $\zeta \in \mathcal{T}_{\mathcal{M}}$, another section, $\operatorname{Lie}_{\zeta} \nu \in \operatorname{Ber}(\mathcal{M})$. As $\nu$ is a basis section, one can write,

$$
\operatorname{Lie}_{\zeta} \nu=(\operatorname{div} \zeta) \nu
$$

for some well-defined function $\operatorname{div} \zeta \in \mathcal{O}_{\mathcal{M}}$ called the divergence of $\zeta$. As the odd 2 -form $\omega$ is non-degenerate, for any function $f \in \mathcal{O}_{\mathcal{M}}$ there exists an associated Hamiltonian vector field, $H_{f} \in \mathcal{T}_{\mathcal{M}}$, uniquely defined by the equation,

$$
d f=\omega\lrcorner H_{f} .
$$

Then one can define an odd differential operator $\Delta$ on $\mathcal{O}_{\mathcal{M}} \simeq \wedge^{\bullet} T_{M}$ as follows,

$$
\Delta f:=\operatorname{div} H_{f},
$$


and check that $\Delta^{2}=0$. If there exist Darboux coordinates, $\left\{x^{\alpha}, \psi_{\alpha}\right\}_{1 \leq \alpha \leq n},\left|\psi_{\alpha}\right|=\left|x^{\alpha}\right|+1$ $\bmod 2 \mathbb{Z}$, on $\mathcal{M}$ such that the Berezinian $\nu$ is locally constant, then the derivation $\Delta$ gets a very simple form,

$$
\Delta=\sum_{\alpha=1}^{n} \frac{\partial^{2}}{\partial x^{\alpha} \partial \psi_{\alpha}}
$$

In this case the data $(\mathcal{M}, \omega, \nu)$ is called an $S P$-manifold [25], and provides us with the most general and down-to-earth mathematical description of key structures in the Batalin-Vilkovisky quantization.

A formal $S P$-manifold can be canonically associated with an arbitrary finite dimensional complex $\left(M_{0}, d_{0}\right)$ over a field $k$. Let $\left\{e_{\alpha}\right\}$ be a homogeneous basis of $M_{0}$ and let $\left\{x^{\alpha}\right\}$ be the dual basis of $M_{0}^{*}$. The differential $d_{0}$ can be understood as a linear odd vector field, $d_{0} \in T_{M}$, on the formal manifold $M$ associated with the vector superspace $M_{0}$. Indeed, if

$$
d_{0}\left(e_{\alpha}\right)=\sum_{\alpha, \beta} D_{\alpha}^{\beta} e_{\beta}, \quad D_{\alpha}^{\beta} \in k
$$

then the associated vector field is given by

$$
\overrightarrow{d_{0}}=\sum_{\alpha \beta}(-1)^{\alpha} x^{\alpha} D_{\alpha}^{\beta} \frac{\partial}{\partial x^{\alpha}} .
$$

Clearly, the equation $d_{0}^{2}=0$ is equivalent to $\left[\vec{d}_{0}, \vec{d}_{0}\right]_{\text {Schouten }}=0$. Hence the $\mathcal{O}_{M}$-module $\wedge^{\bullet} T_{M}$, $\mathcal{O}_{M}:=\widehat{\odot} M_{0}^{*}$ being the ring of formal smooth functions, is naturally a differential $\mathcal{O}_{M}$-module with the differential given by

$$
d f:=\left[d_{0} \bullet f\right]_{\text {Schouten }}
$$

for any $f \in \wedge^{\bullet} T_{M}$. The sheaf $\operatorname{Ber}\left(\Pi \Omega_{M}^{1}\right)$ has a distinguished constant section $\nu$ such that the associated odd Laplacian $\Delta$ has the form (19) with $\psi_{\alpha}=\Pi \partial / \partial x^{\alpha}$. The Schouten brackets on $\mathcal{O}_{\Pi \Omega_{M}^{1}}=\wedge^{\bullet} T_{M}$ get the form,

$$
[f \bullet g]=\sum_{\alpha=1}^{n}\left(\overleftarrow{\frac{\partial}{f} f} \frac{\vec{\partial} g}{\partial x^{\alpha}}+(-1)^{\tilde{g} \tilde{f}} \frac{\overleftarrow{\partial} f}{\partial \psi_{\alpha}^{\alpha}} \frac{\vec{\partial} g}{\partial \psi_{\alpha}}\right)
$$

The resulting data $\left(\wedge^{\bullet} T_{M}, \Delta, d\right)$ is a dGBV algebra canonically associated with the complex $\left(M_{0}, d_{0}\right)$. Hence it makes sense to associate with the latter the following Master equation,

$$
d S+\Delta S+\frac{1}{2}[S \bullet S]=0 .
$$

3.4.3. Theorem. There exists a differential wheeled PROP (PolyV $\left.{ }^{\circlearrowright}, \delta\right)$ whose wheeled representations in a finite dimensional complex $\left(M_{0}, d_{0}\right)$ are in one-to-one correspondence with Master functions $S$ in the $d G B V$ algebra $\left(\wedge^{\bullet} T_{M}, \Delta, d\right)$. 
Proof. Let $E=\left\{E(m, n):=\operatorname{sgn}_{m} \otimes \mathbb{1}_{n}\right\}_{m, n \geq 0}$ be a collection of $\mathbb{Z}_{2}$-graded $\left(\Sigma_{m}, \Sigma_{n}\right)$ bimodules concentrated in degree $m \bmod 2 \mathbb{Z}$. Here $\operatorname{sgn}_{m}$ stands for the sign representation of $\Sigma_{m}$ and $\mathbb{1}_{n}$ for the trivial representation of $\Sigma_{n}$. Every $E(m, n)$ is therefore a one-dimensional space whose basis vector we denote graphically as a planar $(m, n)$-corolla,

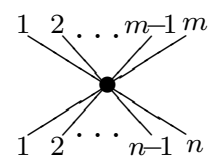

with $m$ skew-symmetric outgoing legs and $n$ symmetric ingoing legs.

Let Poly $\mathrm{V}$ be the free PROP generated by the bimodule $E$, and let Poly $\mathrm{V}^{\circlearrowright}$ be the free wheeled PROP generated by the same bimodule $E$. Any derivation, $\delta$ : Poly $^{\circlearrowright} \rightarrow$ Poly $^{\circlearrowright}$, is completely determined by its values on the above corollas.

Claim. The derivation, $\delta$, defined by

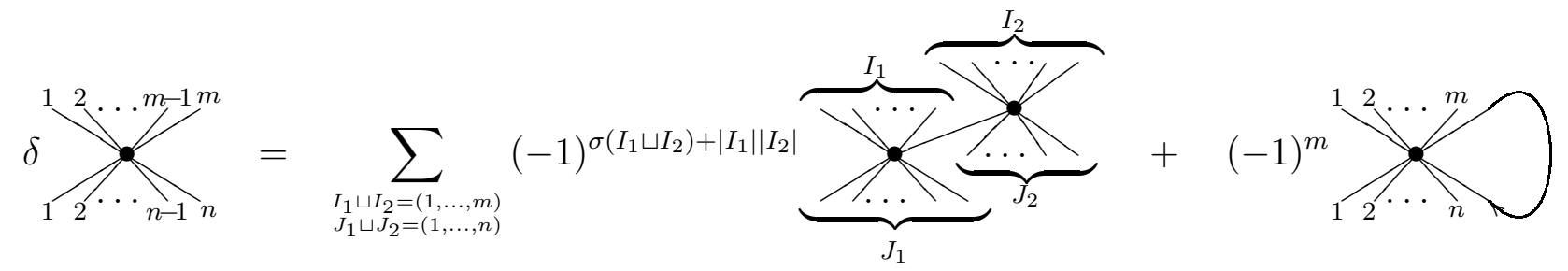

where $\sigma\left(I_{1} \sqcup I_{2}\right)$ is the sign of the shuffle $I_{1} \sqcup I_{2}=(1, \ldots, m)$, is a differential, i.e. $\delta^{2}=0$.

Proof is a straightforward but tedious calculation.

For example

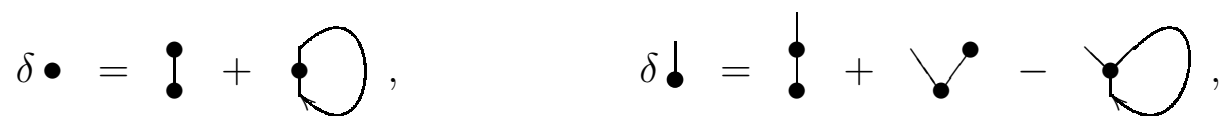

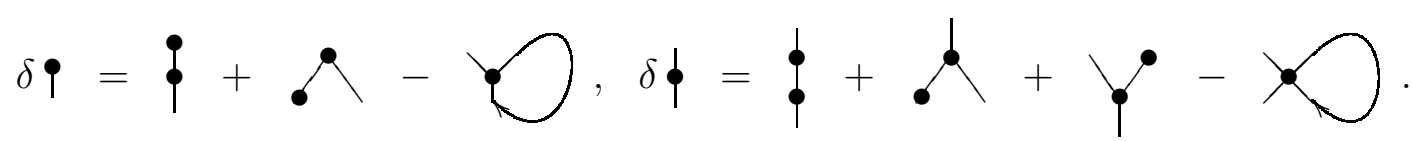

Let us now show that there is an one-to-one correspondence 2 between wheeled representations of Poly $\mathrm{V}^{\circlearrowright}$ and solutions of the Master equations (20). Let

$$
R:\left(\mathrm{PolyV}^{\circlearrowright}, \delta\right) \longrightarrow\left(\mathcal{E}_{n} d_{M_{0}}, d_{0}\right)
$$

be a wheeled representation of Poly $\mathrm{V}^{\circlearrowright}$, in a differential superspace $\left(M_{0}, d_{0}\right)$. If we forget about compatibility with differentials, then, according to Proposition 9, any such a representation is uniquely determined by a representation,

$$
R: \text { PolyV } \longrightarrow \mathcal{E} n d_{M_{0}}
$$

\footnotetext{
${ }^{2}$ In fact this correspondence can be used as another proof of the claim that $\delta$ is a differential, cf. $\S 2.5$ in $[24]$.
} 
which in turn is completely determined by its values, $R_{n}^{m} \in \operatorname{Hom}\left(\odot^{n} M_{0}, \wedge^{m} M_{0}\right)$, on the $(m, n)$ corollas. In the chosen basis $\left\{e_{\alpha}\right\}$ of $M_{0}$ and the associated dual basis $\left\{x^{\alpha}\right\}$ of $M_{0}^{*}$, every such $R_{n}^{m}$ can be decomposed as,

$$
R_{n}^{m}=\sum R_{\beta_{1} \ldots \beta_{n}}^{\alpha_{1} \ldots \alpha_{m}}\left(x^{\beta_{1}} \odot \ldots \odot x^{\beta_{n}}\right) \otimes\left(e_{\alpha_{1}} \wedge \ldots \wedge e_{\alpha_{m}}\right)
$$

for some constants $R_{\beta_{1} \ldots \beta_{n}}^{\alpha_{1} \ldots \alpha_{m}}$. The main idea of the proof is to assemble these constants for all $m, n \geq 0$ into a single generating function, $S$, on the formal supermanifold $\mathcal{M}=\Pi \Omega^{1} M$ with coordinates $x^{\alpha}$ and $\psi_{\beta}:=\prod e_{\beta}$, as follows

$$
S:=\vec{d}_{0}+\sum_{m, n \geq 0} \frac{1}{m ! n !} R_{\beta_{1} \ldots \beta_{n}}^{\alpha_{1} \ldots \alpha_{m}} x^{\beta_{1}} \ldots x^{\beta_{n}} \psi_{\alpha_{1}} \ldots \psi_{\alpha_{m}} \in \mathcal{O}_{\mathcal{M}}
$$

It is now straightforward to check that compatibility of the morphism $R$ with differentials, $R \delta=$ $d_{0} R$, is equivalent to the master equation (20) for $S$.

Thus germs of master functions on $S P$-manifolds are nothing but representations of the differential wheeled PROP (PolyV $\mathrm{C}^{\circlearrowright}, \delta$ ). The class of master functions arising in this way is in a sense typical. Theoretical physicists use its infinite dimensional analogue (with badly defined "volume forms" and divergent contractions of "dual tensors").

3.4.4. Remark. There is an important class of dGBV algebras with the divergence operator $\Delta$ originating from a particular graded metric or symplectic structure on the underlying supermanifold $M$ (i.e., $\Delta$ is a kind of "odd" Laplacian). In this case tangent and cotangent bundles on $M$ are canonically isomorphic so that the associated master equations are better described with the help of modular operads [2] rather than wheeled PROPs.

\section{BAR-Cobar DUAlity FOR WHEELED PROPERAdS}

4.1. Augmentations. The bar and cobar constructions are basic tools to study homological properties of algebraic objects. The bar construction considered in this paper will be a functor from the category of augmented wheeled $\mathrm{dg}$ properads to the category of coaugmented wheeled dg co-properads, and the cobar construction a functor from the category of coaugmented wheeled $\mathrm{dg}$ co-properads to the category of augmented wheeled dg properads. Wheeled properads were introduced in Section 2, In the definition below, $\mathfrak{k}_{c}^{\circlearrowright}$ is the wheeled properad introduced in Example 2.2.2.

4.1.1. Definition. An augmented wheeled properad is a wheeled properad $\mathrm{P}$ together with a homomorphism $\epsilon: \mathrm{P} \rightarrow \mathfrak{k}_{c}^{\circlearrowright}$. The kernel $\overline{\mathrm{P}}:=\operatorname{Ker}(\epsilon) \subset \mathrm{P}$ is the augmentation ideal of $\mathrm{P}$. 
An example is the free wheeled properad $\mathrm{F}^{\circlearrowright}\langle E\rangle$ with the augmentation $\epsilon: \mathrm{F}^{\circlearrowright}\langle E\rangle \rightarrow \mathfrak{k}_{c}^{\circlearrowright}=$ $\mathrm{F}^{\circlearrowright}\langle 0\rangle$ induced by the map $E \rightarrow 0$. Its augmentation ideal equals

$$
\overline{\mathrm{F}}^{\circlearrowright}\langle E\rangle=\underset{G \in \overline{\mathrm{G}}_{c}^{\circlearrowright}(m, n)}{\operatorname{colim}} E(G), m, n \geq 0,
$$

where $\overline{\mathrm{G}}_{c}^{\circlearrowright}(m, n)$ is the category of wheeled directed connected non-exceptional graphs.

Wheeled co-properads are defined by dualizing axioms of wheeled properads. A more precise definition can be given as follows. First, by inverting arrows in Figures 2 and 3 , one recovers the classical notions of cotriple and coalgebras over a cotriple. Next, there exists a cotriple $\mathrm{C}^{\circlearrowright}$ : $\Sigma$-bimod $\rightarrow \Sigma$-bimod whose underlying endofunctor is the same as the underlying endofunctor of the triple $\mathrm{F}^{\circlearrowright}: \Sigma$-bimod $\rightarrow \sum$-bimod in (18), that is

$$
\mathrm{C}^{\circlearrowright}\langle E\rangle(m, n):=\underset{G \in \mathrm{G}_{c}^{\circlearrowright}(m, n)}{\operatorname{colim}} E(G), m, n \geq 0,
$$

Let us indicate how the cotriple structure $\nu: \mathrm{C}^{\circlearrowright} \rightarrow \mathrm{C}^{\circlearrowright} \mathrm{C}^{\circlearrowright}$ can be defined.

Let $E$ be a $\Sigma$-bimodule. As in Section 2, we may imagine elements of $\mathrm{C}^{\circlearrowright}\left\langle\mathrm{C}^{\circlearrowright}\langle E\rangle\right\rangle$ as nested $E$-decorated graphs, with nests encompassing decorated subgraphs that represent elements of the "internal" $\mathrm{C}^{\circlearrowright}\langle E\rangle$. Then, for an element $c \in \mathrm{C}^{\circlearrowright}\langle E\rangle$ represented by a directed graph $G \in \mathrm{G}_{c}^{\circlearrowright}$ with $E$-labeled vertices, $\nu_{E}(c) \in \mathrm{C}^{\circlearrowright}\left\langle\mathrm{C}^{\circlearrowright}\langle E\rangle\right\rangle$ is the sum

$$
\nu_{E}(c)=\sum_{n} c_{n}
$$

taken over all nestings of the graph $G$, with $c_{n}$ being $c$ interpreted as an element of $\mathrm{C}^{\circlearrowright}\left\langle\mathrm{C}^{\circlearrowright}\langle E\rangle\right\rangle$ in the way determined by the nesting $n$. Because $G$ is connected, such nestings are in one-toone correspondence with markings of edges (in the sense introduced below) and an alternative formula for $\nu_{E}(c)$ can be given.

4.1.2. Definition. A marking of $G$ is a map $m: E d g(G) \rightarrow\{\circ, \bullet\}$ from the set of internal edges of $G$ into the two-element set $\{\circ, \bullet\}$. We call edges from $E d g^{\circ}(G):=m^{-1}(\circ)$ white edges and edges from $E d g^{\bullet}(G):=m^{-1}(\bullet)$ black edges.

Suppose we are given a marking $m$ of $G$ as above. Let $G^{\bullet}$ be the graph obtained from $G$ by cutting all white edges in the middle and let $G_{1}^{\bullet}, \ldots, G_{s}^{\bullet}, s \geq 1$, be the connected components of $G^{\bullet}$. The $E$-decoration of vertices of $G$ restricts to decorations of vertices of $G_{i}^{\bullet}$, and these decorated graphs therefore determine elements $c_{i} \in \mathrm{C}^{\circlearrowright}\langle E\rangle, 1 \leq i \leq s$. Let $G^{\circ}$ be the quotient $G / E d g^{\bullet}(G)$ given by contracting black edges of $G$.

Vertices $v_{1}, \ldots, v_{s}$ of $G^{\circ}$ are in one-to-one correspondence with the graphs $G_{1}^{\bullet}, \ldots, G_{s}^{\bullet}$ and each $c_{i}$ induces a $\mathrm{C}^{\circlearrowright}\langle E\rangle$-decoration of the vertex $v_{i} \in E d g\left(G^{\circ}\right), 1 \leq i \leq s$. The graph $G^{\circ}$ with this decoration then determines an element $c_{m} \in \mathrm{C}^{\circlearrowright}\left\langle\mathrm{C}^{\circlearrowright}\langle E\rangle\right\rangle$. One has

$$
\nu_{E}(c):=\sum_{m} c_{m}
$$


where the sum runs over all markings of the graph $G$.

4.1.3. Definition. Wheeled co-properads are coalgebras over the cotriple $\mathrm{C}^{\circlearrowright}$ : $\Sigma$-bimod $\rightarrow$ $\Sigma$-bimod.

Observe that, for each $\Sigma$-bimodule $E, \mathrm{C}^{\circlearrowright}\langle E\rangle$ is a wheeled co-properad, with the co-properad structure given by the cotriple map $\nu_{E}: \mathrm{C}^{\circlearrowright}\langle E\rangle \rightarrow \mathrm{C}^{\circlearrowright}\left\langle\mathrm{C}^{\circlearrowright}\langle E\rangle\right\rangle$.

Important examples of wheeled co-properads can be obtained by taking linear duals of wheeled properads satisfying a mild finiteness assumption. We say that a graded wheeled (co-)properad $\mathrm{P}$ is of finite type if the graded space $\mathrm{P}(m, n)$ is of finite type for each $m, n \geq 0$. The (componentwise) linear duals of wheeled properads of finite type are wheeled co-properads, and the linear duals of wheeled co-properads of finite type are wheeled properads, compare Remark II.3.4 of [19]. We denote the linear dual of a wheeled (co-)properad P by P\#.

The linear dual $\mathfrak{k}_{c}^{\circlearrowright \#}$ of the trivial wheeled properad $\mathfrak{k}_{c}^{\circlearrowright}$ turns out to be the terminal object of the category of co-properads. Observe that $\mathfrak{k}_{c}^{\circlearrowright \#}=\mathrm{C}^{\circlearrowright}\langle 0\rangle$.

4.1.4. Definition. A coaugmented wheeled co-properad is a wheeled co-properad C equipped with a homomorphism $\eta: \mathfrak{k}_{c}^{\circlearrowright \#} \rightarrow C$. Its coaugmentation coideal is the coimage $\bar{C}:=\operatorname{Coim}(\eta)$.

The wheeled co-properad $\mathrm{C}^{\circlearrowright}\langle E\rangle$ is coaugmented, with the coaugmentation $\eta: \mathfrak{k}_{c}^{\circlearrowright} \rightarrow \mathrm{C}^{\circlearrowright}\langle E\rangle$ induced by the map $0 \rightarrow E$. Its coaugmentation coideal equals

$$
\overline{\mathrm{C}}^{\circlearrowright}\langle E\rangle=\underset{G \in \overline{\mathrm{G}}_{c}^{\circlearrowright}(m, n)}{\operatorname{colim}} E(G), m, n \geq 0,
$$

where $\overline{\mathrm{G}}_{c}^{\circlearrowright}(m, n)$ is as in (21) .

The wheeled suspension of a $\Sigma$-bimodule $E=\{E(m, n)\}_{m, n \geq 0}$ is a $\Sigma$-bimodule

$$
\mathbf{w} E=\{\mathbf{w} E(m, n)\}_{m, n \geq 0}
$$

with components

$$
\mathbf{w} E(m, n)=\uparrow^{2 m-n} E(m, n) \otimes \operatorname{sgn}_{n}, m, n \geq 0 .
$$

Its wheeled desuspension $\mathbf{w}^{-1} E$ is defined by a similar formula:

$$
\mathbf{w}^{-1} E(m, n)=\downarrow^{2 m-n} E(m, n) \otimes \operatorname{sgn}_{n}, \quad \text { for } m, n \geq 0 .
$$

The origin of the above formulas will be explained in Remark 4.2 .2 below. 
4.2. Bar and cobar. We are ready to introduce the bar construction. Let $P=\left(P, \partial_{P}\right)$ be an augmented wheeled dg properad with a degree 1 differential $\partial_{\mathrm{P}}$. Consider the wheeled co-properad $\mathrm{C}^{\circlearrowright}\left\langle\mathbf{w}^{-1} \overline{\mathrm{P}}\right\rangle$ cogenerated by the $\Sigma$-bicollection $\mathbf{w}^{-1} \overline{\mathrm{P}}$, where $\overline{\mathrm{P}}$ is the augmentation ideal of $\mathrm{P}$. The differential $\partial_{\mathrm{P}}$ of $\mathrm{P}$ induces, in the standard way, a degree 1 coderivation $\partial_{1}$ of $\mathrm{C}^{\circlearrowright}\left\langle\mathbf{w}^{-1} \overline{\mathrm{P}}\right\rangle$. The structure operation $\mathrm{F}^{\circlearrowright}\langle\overline{\mathrm{P}}\rangle \rightarrow \mathrm{P}$ of the wheeled properad $\mathrm{P}$ induces, precisely as in the operadic case (see [17]), another degree 1 coderivation $\partial_{2}$ on $C^{\circlearrowright}\left\langle\mathbf{w}^{-1} \bar{P}\right\rangle$. It is not difficult to show that both $\partial_{1}$ and $\partial_{2}$ are differentials commuting with each other.

4.2.1. Definition. The wheeled dg co-properad $\left(\mathrm{C}^{\circlearrowright}\left\langle\mathbf{w}^{-1} \overline{\mathrm{P}}\right\rangle, \partial_{B}\right)$ with $\partial_{B}:=\partial_{1}+\partial_{2}$ will be called the bar construction of $\mathrm{P}$ and denoted $B^{\circlearrowright}(\mathrm{P})=\left(B^{\circlearrowright}(\mathrm{P}), \partial_{B}\right)$.

4.2.2. Remark. Definition 4.2.1 is a modification of the bar construction (called the Feynman transform) of a modular (co)operad, see [19, Definition II.5.58] or the original source [8]. Unlike the bar construction of an ordinary operad, the Feynman transform of a modular operad is a 'twisted' modular operad. The twisting is specified by the dualizing cocycle $\mathfrak{K}$ [19, Example II.5.52]

$$
\mathfrak{K}(G):=\downarrow^{|E d g(G)|} \operatorname{Det}(E d g(G))=\downarrow^{|E d g(G)|} \Lambda^{|E d g(G)|}(\langle E d g(G)\rangle),
$$

the determinant of the span of the set $E d g(G)$ of internal edges of $G$, placed in degree $-|E d g(G)|$.

When $G$ is directed, $\operatorname{Edg}(G)$ is clearly canonically isomorphic to the union $\bigcup_{v \in \operatorname{Vert}(G)}$ out $(v)$ of outgoing edges of vertices of $G$, minus the set of outgoing legs of $G$. Consequently, on directed graphs, $\mathfrak{K}$ is a coboundary (in the sense of [19, Lemma II.5.49]) and hence its action on a decorated graph $G$ is equivalent to the tensor product of the decoration $\Sigma$-bimodule $E(m, n)$ with the $\Sigma$-bimodule $u(m, n):=\operatorname{sgn}_{m} \otimes \downarrow^{m} \mathbf{k} \otimes \mathbb{1}_{n}$.

The next step is to modify the degrees in such a way that the bar construction of the quadratic dual of a wheeled Koszul properad will be concentrated in degree zero. This can be achieved by taking the tensor product with the desuspension wheeled properad $\mathrm{S}^{-1}$ (see \$2.1.11),

$$
\left(\operatorname{sgn}_{m} \otimes \downarrow^{m} \mathbf{k} \otimes \mathbb{1}_{n}\right) \otimes\left(\operatorname{sgn}_{m} \otimes \downarrow^{m-n} \mathbf{k} \otimes \operatorname{sgn}_{n}\right)=\mathbb{1}_{m} \otimes \downarrow^{2 m-n} \mathbf{k} \otimes \operatorname{sgn}_{n} .
$$

We recognize the formula for the wheeled desuspension $\mathbf{w}^{-1}$ which we use in the bar construction.

Let us look more closely at the structure of $B^{\circlearrowright}(\mathrm{P})$. Elements of $B^{\circlearrowright}(\mathrm{P})(m, n)$ are represented by linear combinations of graphs $G \in \mathrm{G}_{c}^{\circlearrowright}(m, n)$ with vertices decorated by appropriately desuspensed elements of $\overline{\mathrm{P}}$. The degree of an element $x \in B^{\circlearrowright}(\mathrm{P})(m, n)$ with the underlying graph $G$ is

$$
\sum_{v \in \operatorname{Vert}(G)} \operatorname{deg}\left(p_{v}\right)-2 m+n-e(G),
$$


where $e(G)$ is the number of internal edges of $G$ and $\operatorname{deg}\left(p_{v}\right)$ the degree of the decoration $p_{v} \in \overline{\mathrm{P}}$ of a vertex $v$ of $G$. The differential $\partial_{1}(x)$ decomposes into the sum

$$
\partial_{1}(x)=\sum_{v \in \operatorname{Vert}(G)} \epsilon^{v} \partial_{v}(x)
$$

where $\partial_{v}$ replaces the decoration $p_{v}$ of $v$ by $\partial_{\mathrm{P}}\left(p_{v}\right)$ and $\epsilon^{v}$ is an appropriate sign. Likewise, $\partial_{2}(v)$ decomposes as

$$
\partial_{2}(x)=\sum_{e \in E d g(G)} \epsilon^{e} \partial_{e}(x)
$$

where $\partial_{e}$ acts as follows.

If the edge $e$ connects two different vertices, then $\partial_{e}$ contracts $e$ and decorates the new vertex obtained by contracting $e$ by the properadic composition of the decorations of the vertices connected by $e$. If $e$ is a directed loop starting and ending in the same vertex, then $\partial_{e}$ removes $e$ and decorates the vertex by the corresponding contraction of the original decoration. As before, $\epsilon^{e}$ is an appropriate sign.

The cobar construction is defined in the dual manner. For a wheeled dg coaugmented coproperad $\mathrm{C}=\left(\mathrm{C}, \partial_{\mathrm{C}}\right)$, we consider the free wheeled properad $\mathrm{F}^{\circlearrowright}\langle\mathbf{w} \overline{\mathrm{C}}\rangle$ generated by the wheeled suspension of the coaugmentation coideal of $\mathrm{C}$. The differential $\partial_{\mathrm{C}}$ induces a degree 1 derivation $\partial_{1}$ and the structure operations of the co-properad $C$ a degree 1 derivation $\partial_{2}$. As before, both $\partial_{1}$ and $\partial_{2}$ are differentials that commute with each other.

4.2.3. Definition. The wheeled dg properad $\left(\mathrm{F}^{\circlearrowright}\langle\mathbf{w} \overline{\mathrm{C}}\rangle, \partial_{\Omega}\right)$ with $\partial_{\Omega}:=\partial_{1}+\partial_{2}$ will be called the cobar construction of $\mathrm{C}$ and denoted $\Omega^{\circlearrowright}(\mathrm{C})=\left(\Omega^{\circlearrowright}(\mathrm{C}), \partial_{\Omega}\right)$.

The bar construction of Definition 4.2.1 clearly extends to a functor

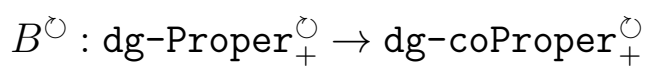

from the category of augmented wheeled dg-properads to the category of augmented wheeled dg-co-properads, while the cobar construction of Definition 4.2 .3 extends to a functor

$$
\Omega^{\circlearrowright}: \mathrm{dg} \text {-coProper }{ }_{+}^{\circlearrowright} \rightarrow \mathrm{dg} \text {-Proper }{ }_{+}^{\circlearrowright}
$$

The following proposition shows that $B^{\circlearrowright}$ and $\Omega^{\circlearrowright}$, restricted to suitable subcategories, are exact functors.

4.2.4. Proposition. Let $\alpha: \mathrm{P}^{\prime} \rightarrow \mathrm{P}^{\prime \prime}$ be a homology isomorphism of non-positively graded augmented dg properads. Then the induced map $B^{\circlearrowright}(\alpha): B^{\circlearrowright}\left(\mathrm{P}^{\prime}\right) \rightarrow B^{\circlearrowright}\left(\mathrm{P}^{\prime \prime}\right)$ of the bar constructions is a homology isomorphism, too.

Similarly, the map $\Omega^{\circlearrowright}(\beta): \Omega^{\circlearrowright}\left(\mathrm{C}^{\prime}\right) \rightarrow \Omega^{\circlearrowright}\left(\mathrm{C}^{\prime \prime}\right)$ induced by a homology isomorphism $\beta: \mathrm{C}^{\prime} \rightarrow \mathrm{C}^{\prime \prime}$ of non-negatively graded $d g$ coaugmented co-properads is also a homology isomorphism. 
Proof. The proposition follows from a simple spectral sequence argument based on the filtration given by the number of edges of the underlying graphs. The assumption about the non-positivity (resp. non-negativity) of the grading guarantees that the induced spectral sequences converge.

The following theorem is a key technical ingredient in showing that $B^{\circlearrowright}$ and $\Omega^{\circlearrowright}$ restricted to suitable subcategories are mutual homology inverses.

4.2.5. Theorem. For each $d g$ wheeled augmented properad $\mathrm{P}$, the natural projection of wheeled dg properads $\pi: \Omega^{\circlearrowright}\left(B^{\circlearrowright}(\mathrm{P})\right) \rightarrow \mathrm{P}$ is a quasi-isomorphism.

Dually, for a wheeled coaugmented dg co-properad $\mathrm{C}$, the natural inclusion $\iota: \mathrm{C} \hookrightarrow B^{\circlearrowright}\left(\Omega^{\circlearrowright}(\mathrm{C})\right)$ of wheeled dg co-properads is a quasi-isomorphism.

Proof. Let us first explicitly describe the epimorphism $\pi$. As a non-dg properad, $\Omega^{\circlearrowright}\left(B^{\circlearrowright}(\mathrm{P})\right)$ is free, generated by the $\Sigma$-bimodule $\mathbf{w} \bar{C}^{\circlearrowright}\left\langle\mathbf{w}^{-1} \overline{\mathrm{P}}\right\rangle$. Therefore each wheeled properad homomorphism $\Omega^{\circlearrowright}\left(B^{\circlearrowright}(\mathrm{P})\right) \rightarrow \mathrm{P}$ is determined by a map $\mathbf{w} \overline{\mathrm{C}}^{\circlearrowright}\left\langle\mathbf{w}^{-1} \overline{\mathrm{P}}\right\rangle \rightarrow \mathrm{P}$ of $\Sigma$-bimodules. We define $\pi$ as the homomorphism corresponding to the composition

$$
\mathbf{w} \overline{\mathrm{C}}^{\circlearrowright}\left\langle\mathbf{w}^{-1} \overline{\mathrm{P}}\right\rangle \stackrel{\mathbf{w}(p)}{\longrightarrow} \overline{\mathrm{P}} \hookrightarrow \mathrm{P},
$$

where $\mathbf{w}(p)$ is the wheeled suspension of the projection $p: \overline{\mathrm{C}}^{\circlearrowright}\left\langle\mathbf{w}^{-1} \overline{\mathbf{P}}\right\rangle \rightarrow \mathbf{w}^{-1} \overline{\mathbf{P}}$ to the space of cogenerators. We will show that the homology of $\left(\Omega^{\circlearrowright}\left(B^{\circlearrowright}(\mathrm{P})\right), \partial_{\Omega}\right)$ is isomorphic to the homology of P. It will be clear from our proof that this isomorphism is induced by the map $\pi$ constructed above.

Fix $m, n \geq 0$. As before, elements of $\left(\Omega^{\circlearrowright}\left(B^{\circlearrowright}(\mathrm{P})\right)(m, n)=\mathrm{F}^{\circlearrowright}\left\langle\mathbf{w} \overline{\mathrm{C}}^{\circlearrowright}\left\langle\mathbf{w}^{-1} \overline{\mathrm{P}}\right\rangle\right\rangle(m, n)\right.$ are represented by nested graphs $G \in \mathrm{G}_{c}^{\circlearrowright}(m, n)$ with vertices decorated by appropriate (de)suspensions of elements of $\overline{\mathrm{P}}$. Since $G$ is connected, its nestings can be equivalently described by markings $m: \operatorname{Edg}(G) \rightarrow\{\circ, \bullet\}$. The connected components of the graph $G^{\bullet}$ obtained from $G$ by cutting all white edges in the middle, with the induced decoration of vertices, determine elements of $\overline{\mathrm{C}}^{\circlearrowright}\left\langle\mathbf{w}^{-1} \overline{\mathrm{P}}\right\rangle$ whose suspensions decorate vertices of the quotient $G^{\circ}:=G / E d g^{\bullet}(G)$. The degree of an element with the underlying marked graph $G$ equals

$$
-e^{\bullet}(G)+\sum_{v \in \operatorname{Vert}(G)} \operatorname{deg}\left(p_{v}\right),
$$

where $e^{\bullet}(G)$ is the number of black edges of $G$ and $\operatorname{deg}\left(p_{v}\right)$ the degree of the decoration $p_{v} \in \overline{\mathrm{P}}$ of a vertex $v \in \operatorname{Vert}(G)$.

Recall that the differential $\partial_{\Omega}$ of $\Omega^{\circlearrowright}\left(B^{\circlearrowright}(\mathrm{P})\right)$ decomposes as $\partial_{\Omega}=\partial_{\mathrm{P}}+\partial_{1}+\partial_{2}$, where $\partial_{\mathrm{P}}$ is the differential induced by the differential in $\mathrm{P}$, the sum $\partial_{\mathrm{P}}+\partial_{1}$ is the differential induced by the differential $\partial_{B}$ of $B^{\circlearrowright}(\mathrm{P})$, and $\partial_{2}$ is induced by the co-properad structure of $B^{\circlearrowright}(\mathrm{P})$. Obviously the part $\partial_{\mathbf{P}}+\partial_{1}$ of the total differential does not change the number of white edges, while $\partial_{2}$ 
increases it by one. In fact, the differential $\partial_{2}$ applied to an element $x \in \Omega^{\circlearrowright}\left(B^{\circlearrowright}(\mathrm{P})\right)$ with the underlying marked graph $G$ equals

$$
\partial_{2}(x)=\sum_{e \in E d g^{\bullet}(G)} \epsilon^{e} \partial_{e}(x)
$$

where $\partial_{e}$ changes the color of $e$ from black to white, and $\epsilon^{e}$ is an appropriate sign.

Let us consider the spectral sequence $E=\left\{\left(E^{r}, d^{r}\right)\right\}_{r \geq 0}$ induced by an increasing filtration of the complex $\Omega^{\circlearrowright}\left(B^{\circlearrowright}(\mathrm{P})\right)$

$$
0 \subset F_{0} \subset F_{1} \subset \ldots F_{k} \subset F_{k+1} \subset \ldots
$$

with $F_{k}$ being the subspace of $\Omega^{\circlearrowright}\left(B^{\circlearrowright}(\mathrm{P})\right)$ spanned by decorated graphs, $G$, with at most $k$ total number of internal edges, i.e.

number of black edges of $G+$ number of white edges of $G \leq k$.

As the filtration is bounded below and exhaustive, the spectral sequence $E=\left\{\left(E^{r}, d^{r}\right)\right\}_{r \geq 0}$ converges to the cohomology of $\Omega^{\circlearrowright}\left(B^{\circlearrowright}(\mathrm{P})\right)$. To prove the first part of the Theorem we have to show that this cohomology equals to the cohomology of the wheeled properad $\mathrm{P}$. The 0th term of this spectral sequence, $\left(E^{0}, d^{0}\right)$ has the differential $d^{0}=\partial_{\mathrm{P}}+\partial_{2}$. To compute $E^{1}$ we notice that the complex $E^{0}$ sptits into a direct sum

$$
E^{0}=\bigoplus_{[G] \in\left[\mathrm{G}_{c}^{\circlearrowright}\right]} \Omega^{\circlearrowright}\left(B^{\circlearrowright}(\mathrm{P})\right)_{[G]},
$$

where $\left[\mathrm{G}_{c}^{\circlearrowright}\right]$ is the set of isomorphism classes of graphs in $\mathrm{G}_{c}^{\circlearrowright}$ and $\Omega^{\circlearrowright}\left(B^{\circlearrowright}(\mathrm{P})\right)_{[G]}$ is the subspace of $\Omega^{\circlearrowright}\left(B^{\circlearrowright}(\mathrm{P})\right)$ spanned by elements with the underlying graph isomorphic to $G$. The differential $d^{0}=\partial_{\mathrm{P}}+\partial_{2}$ clearly preserves this decomposition. Thus to compute $E^{1}$ it is enough to compute cohomology of the complex

$$
\left(E_{G}^{0}:=\Omega^{\circlearrowright}\left(B^{\circlearrowright}(\mathrm{P})\right)_{[G]}, d_{\mathrm{P}}+\partial_{2}\right)
$$

for any particular fixed $(m, n)$-graph $G$. This complex is actually a bicomplex, $E_{G}^{0}=\left\{E_{G}^{p, q}\right\}$ with $p$ being the total $\mathrm{P}$-degree of $\mathrm{P}$-decorate vertices, and $q$ is the number of white vertices. Notice that $q$ ranges from zero to the total number of edges in $G$.

The filtration of the complex $E_{G}^{0}$ by the number of white vertices is bounded, hence the induced spectral sequence $\left(\mathcal{E}_{G}^{r}, \delta^{r}\right)$ converges to the cohomology of $E_{G}^{0}$. The 0 th term, $\mathcal{E}_{G}^{0}$, of this sequence has the differential $\delta^{0}$ equal to $d_{\mathrm{P}}$. Hence $\mathcal{E}_{G}^{1}$ is spanned by graphs $G$ decorated with elements of the cohomology wheeled properad $H(\mathrm{P})$, and $\delta^{1}$ is equal to $\partial_{2}$, the differential in $\Omega^{\circlearrowright}\left(B^{\circlearrowright}(H(\mathrm{P}))\right)$ coding the coproperad structure in $B^{\circlearrowright}(H(\mathrm{P}))$.

To compute $\mathcal{E}_{G}^{2}=H\left(\mathcal{E}_{G}^{1}, \delta^{1}\right)$ for a non-exceptional graph $G \in \mathrm{G}_{c}^{\circlearrowright}(m, n)$, let $\left\{e_{1}, \ldots, e_{s}\right\}=$ $\operatorname{Edg}(G)$ be the set of edges of $G$. It is a simple exercise to prove that the exterior algebra

$$
\left(\wedge\left(e_{1}, \ldots, e_{s}\right), \partial_{\wedge}\right), \operatorname{deg}\left(e_{i}\right):=-1 \text { for } 1 \leq i \leq s,
$$


with the differential $\partial_{\wedge}:=\sum_{1 \leq i \leq s} \partial / \partial e_{i}$, is acyclic whenever $s \geq 1$. Observe also that $\partial_{\wedge}$ is equivariant under the action of the symmetric group of $\left\{e_{1} \ldots, e_{s}\right\}$. The acyclicity of (24) implies that also the product

$$
\left(\overline{H(\mathrm{P})}(G) \otimes \wedge\left(e_{1}, \ldots, e_{s}\right), \partial\right)
$$

in which $\overline{H(\mathrm{P})}(G)$ is as in (10) and $\partial(\bar{x} \otimes u):=(-1)^{\operatorname{deg}(\bar{x})} \bar{x} \otimes \partial_{\wedge} u$, for $\bar{x} \in \overline{H(\mathrm{P})}(G)$ and $u \in \wedge\left(e_{1}, \ldots, e_{s}\right)$, is acyclic.

The group $\operatorname{Aut}(G)$ of automorphisms of $G$ acts on $\wedge\left(e_{1}, \ldots, e_{s}\right)$ by permutations of edges, which implies that $\partial_{\wedge}$ is $A u t(G)$-equivariant. Therefore also the differential $\partial$ on the product (25) is $A u t(G)$-equivariant, hence it induces a differential $\partial_{G}$ on the orbit space

$$
\left(\overline{H(\mathrm{P})}(G) \otimes \wedge\left(e_{1}, \ldots, e_{s}\right)\right)_{G}:=\left(\overline{H(\mathrm{P})}(G) \otimes \wedge\left(e_{1}, \ldots, e_{s}\right)\right) / A u t(G) .
$$

Since $\operatorname{Aut}(G)$ is a finite group and the ground field has characteristic zero, the acyclicity of (25) implies the acyclicity of $\left.\left(\overline{H(\mathrm{P})}(G) \otimes \wedge\left(e_{1}, \ldots, e_{s}\right)\right)_{G}, \partial\right)$, for each $s \geq 1$.

There is an isomorphism

$$
\Psi_{G}:\left(\Omega^{\circlearrowright}\left(B^{\circlearrowright}(H(\mathrm{P}))\right)_{[G]}, \partial_{2}\right) \stackrel{\cong}{\rightrightarrows}\left(\left(\overline{H(\mathrm{P})}(G) \otimes \wedge\left(e_{1}, \ldots, e_{s}\right)\right)_{G}, \partial_{G}\right)
$$

that sends the isomorphism class of an element $x \in \Omega^{\circlearrowright}\left(B^{\circlearrowright}(H(\mathrm{P}))\right)_{[G]}$ represented by a $\overline{H(\mathrm{P})}$ decorated graph $G$ with marking $m$, into the orbit of

$$
\bar{x} \otimes\left(e_{i_{1}} \wedge \cdots \wedge e_{i_{t}}\right) \in \overline{H(\mathrm{P})}(G) \otimes \wedge\left(e_{1}, \ldots, e_{s}\right),
$$

where $\bar{x} \in \overline{H(\mathrm{P})}(G)$ is obtained from $x$ by forgetting the marking of $G$, and

$$
\left\{e_{i_{1}}, \ldots, e_{i_{t}}\right\}=E d g^{\bullet}(G), i_{1}<\cdots<i_{t} .
$$

It is easy to see that $\Psi$ is well-defined and that it commutes with the differentials. Its inverse

$$
\Psi_{G}^{-1}:\left(\left(\overline{H(\mathrm{P})}(G) \otimes \wedge\left(e_{1}, \ldots, e_{s}\right)\right)_{G}, \partial_{G}\right) \stackrel{\cong}{\rightrightarrows}\left(\Omega^{\circlearrowright}\left(B^{\circlearrowright}(H(\mathrm{P}))\right)_{[G]}, \partial_{2}\right)
$$

maps $\bar{x} \otimes e_{i_{1}} \wedge \cdots \wedge e_{i_{t}}$, where $\bar{x} \in \overline{H(\mathrm{P})}(G)$ is represented by a $\overline{H(\mathrm{P})}$-decoration of $G$, into $x \in \Omega^{\circlearrowright}\left(B^{\circlearrowright}(H(\mathrm{P}))\right)_{[G]}$ given by the same $\overline{H(\mathrm{P})}$-decorated graph as $\bar{x}$ but with marking defined by (27).

We conclude from (26) that $\Omega^{\circlearrowright}\left(B^{\circlearrowright}(H(\mathrm{P}))\right)_{[G]}$ is $\partial_{2}$-acyclic whenever $G$ has at least one internal edge. If $G$ has no internal edge, i e. when $G$ is an $(m, n)$-corolla, then clearly

$$
H\left(\Omega^{\circlearrowright}\left(B^{\circlearrowright}(H(\mathrm{P}))\right)_{[G]}, \partial_{2}\right) \cong \overline{H(\mathrm{P})}(G)(m, n) .
$$

The two exceptional graphs $\uparrow$ and $\circlearrowright$ contribute to (23) by $\mathfrak{k}_{c}^{\circlearrowright}(1,1)$ if $(m, n)=(1,1)$ and by $\mathfrak{k}_{c}^{\circlearrowright}(0,0)$ if $(m, n)=(0,0)$. As all the higher differentials in both of our spectral sequences are obviously zero, we obtain an isomorphism,

$$
H\left(\Omega^{\circlearrowright}\left(B^{\circlearrowright}(\mathrm{P})\right)\right) \cong \overline{H(\mathrm{P})} \oplus \mathfrak{k}_{c}^{\circlearrowright} \cong H(\mathrm{P})
$$


proving the first claim in the Theorem that the surjection $\pi$ is a quasi-isomorphism.

The second claim on the map $\iota: \mathrm{C} \rightarrow B^{\circlearrowright}\left(\Omega^{\circlearrowright}(\mathrm{C})\right)$ can be proven by dualizing the above arguments.

\section{Wheeled operads, quadratic Duality and Koszulness}

5.1. Wheeled operads. As operads form a subcategory of PROPs [15], there is a similar subcategory of wheeled operads in the category of wheeled PROPs. In this section we introduce these wheeled operads, define their quadratic duals and study their Koszulness. Methods of this section will provide a conceptual understanding of minimal models of wheeled completions of classical quadratic operads as Ass, Lie or Com.

5.1.1. Definition. A wheeled operad is a wheeled properad $\mathcal{P}=\{\mathcal{P}(m, n)\}_{m, n \geq 0}$, in the sense of Definition 2.2.1, such that $\mathcal{P}(m, n)=0$ whenever $m \geq 2$.

It is obvious from this definition that a wheeled operad $\mathcal{P}=\{\mathcal{P}(m, n)\}_{m, n \geq 0}$ consists of

(i) an ordinary operad $\mathcal{P}_{o}:=\{\mathcal{P}(1, n)\}_{n \geq 0}$,

(ii) a right $\mathcal{P}_{o^{-}}$module $\mathcal{P}_{w}:=\{\mathcal{P}(0, n)\}_{n \geq 0}$ (see [19, Def. II.3.26] for a definition of right operadic modules), and

(iii) contractions $\xi_{i}: \mathcal{P}_{o}(n) \rightarrow \mathcal{P}_{w}(n-1), 1 \leq i \leq n$, that are compatible, in the appropriate sense, with the structures in (i) and (ii).

5.1.2. Definition. The operad $\mathcal{P}_{o}$ and the right $\mathcal{P}_{o}$-module $\mathcal{P}_{w}$ defined above are called the operadic and wheeled parts of the wheeled operad $\mathcal{P}$, respectively.

Wheeled properadic completions of ordinary operads provide examples of wheeled operads, but, as we will see immediately, not all wheeled operads are of this form. Recall [19, Definition II.3.31] that an ordinary operad $\mathcal{P}$ is quadratic if it is of the form

$$
\mathcal{P}=\Gamma_{o p}\langle E\rangle / I
$$

where $\Gamma_{o p}\langle E\rangle$ is the free operad on a right $\Sigma_{2}$-module considered as a $\Sigma$-module with

$$
E(n):= \begin{cases}E, & \text { if } n=2 \text { and } \\ 0, & \text { otherwise, }\end{cases}
$$

and $I$ an operadic ideal generated by a subspace $R \subset \Gamma_{o p}\langle E\rangle(3)$. The quadratic dual $\mathcal{P}^{\text {! }}$ of a quadratic operad $\mathcal{P}$ is defined as

$$
\mathcal{P}^{!}:=\Gamma_{o p}\left\langle E^{\vee}\right\rangle /\left(R^{\perp}\right),
$$

where $E^{\vee}:=\operatorname{Lin}(E, \mathbf{k}) \otimes \operatorname{sgn}_{2}$ is the Czech dual of $E$ and $R^{\perp}$ the annihilator of $R$ in $\Gamma_{o p}\left\langle E^{\vee}\right\rangle(3) \cong$ $\Gamma_{o p}\langle E\rangle(3)^{\vee}$. See [19, Definition II.3.37] for details. Let us introduce wheeled versions of the above notions. 
5.1.3. Definition. Quadratic wheeled operad is a wheeled operad $\mathcal{P}$ of the form

$$
\mathcal{P}=\mathrm{F}^{\circlearrowright}\langle E\rangle / I
$$

where $E$ is a left $\Sigma_{1}$ - right $\Sigma_{2}$-bimodule considered as a $\Sigma$-bimodule with

$$
E(m, n):= \begin{cases}E, & \text { if }(m, n)=(1,2) \text { and } \\ 0, & \text { otherwise, }\end{cases}
$$

and $I$ a wheeled operadic ideal generated by a subspace

$$
R_{o} \oplus R_{w} \subset \mathrm{F}^{\circlearrowright}\langle E\rangle(1,3) \oplus \mathrm{F}^{\circlearrowright}\langle E\rangle(0,1) .
$$

We will always assume that $E$ is finite dimensional.

In the rest of this paper, we will often work with the wheeled properadic completion of operads. To simplify the terminology and notation, we will call it simply the wheeled completion and drop the subscript from the notation, that is, write $\mathcal{P}^{\circlearrowright}$ instead of $\mathcal{P}_{c}^{\circlearrowright}$. We believe that no confusion is possible.

5.1.4. Example. A wheeled quadratic operad is the wheeled completion of an ordinary quadratic operad if and only if the space $R_{w}$ in (30) is trivial. If $\mathcal{P}$ is an ordinary quadratic operad as in (28) with $I$ generated by a subspace $R \subset \Gamma_{o p}\langle E\rangle(3)$, then $\mathcal{P} \circlearrowright$ is wheeled quadratic with the same space of generators and with $R_{o}=R, R_{w}=0$.

In particular, wheeled completions $\mathrm{Ass}^{\circlearrowright}, \mathrm{Com}^{\circlearrowright}$ and $\mathrm{Lie}^{\circlearrowright}$ of quadratic operads Ass, Com and Lie for associative, commutative associative and Lie algebras, respectively, are wheeled quadratic operads. The initial wheeled properad $\mathfrak{k}^{\mho}$ is a wheeled quadratic operad generated by the trivial $\Sigma$-bimodule. Therefore every wheeled quadratic operad $\mathcal{P}$ is augmented, with the augmentation $\mathcal{P} \rightarrow \mathfrak{k}^{\circlearrowright}$ induced by the map $E \rightarrow 0$ of generators.

5.2. Quadratic duality and Koszulness. We need to extend the definition of the Czech dual to $\Sigma$-bimodules. For a graded left $\mathbf{k}\left[\Sigma_{m}\right]$ - right $\mathbf{k}\left[\Sigma_{n}\right]$-bimodule $U$ define $U^{\vee}$ by

$$
\left(U^{\vee}\right)^{i}:=\operatorname{sgn}_{m} \otimes \operatorname{Lin}\left(U_{-i}, \mathbf{k}\right) \otimes \operatorname{sgn}_{n},
$$

where $\operatorname{Lin}\left(U_{-i}, \mathbf{k}\right)$ is the ordinary linear dual of $U_{-i}$ and sgn the signum representation. The Czech dual of a $\Sigma$-bimodule is then the componentwise application of the above operation.

As in the operadic case (see [19, Section II.3.2]) there is, for each $\Sigma$-bimodule $E$, a natural biequivariant isomorphism $\mathrm{F}^{\circlearrowright}\left\langle E^{\vee}\right\rangle \cong \mathrm{F}^{\circlearrowright}\langle E\rangle^{\vee}$. Therefore the annihilator of each subspace $S \subset$ $\mathrm{F}^{\circlearrowright}\langle E\rangle(m, n)$ can be regarded as a subspace $S^{\perp} \subset \mathrm{F}^{\circlearrowright}\left\langle E^{\vee}\right\rangle(m, n), m, n \geq 0$.

5.2.1. Definition. Let $\mathcal{P}$ be a wheeled quadratic operad as in Definition 5.1.3. Define its wheeled quadratic dual as the wheeled quadratic operad

$$
\mathcal{P}^{!}:=\mathrm{F}^{\circlearrowright}\left\langle E^{\vee}\right\rangle / I^{\perp}
$$


with $I^{\perp}$ the ideal generated by

$$
R_{o}^{\perp} \oplus R_{w}^{\perp} \subset \mathrm{F}^{\circlearrowright}\left\langle E^{\vee}\right\rangle(1,3) \oplus \mathrm{F}^{\circlearrowright}\left\langle E^{\vee}\right\rangle(0,1)
$$

The wheeled quadratic dual is clearly an involution in the category of wheeled quadratic operads, $\left(\mathcal{P}^{!}\right)^{!}=\mathcal{P}$. Let us prove the following simple but useful proposition.

5.2.2. Proposition. Let $\mathcal{P}$ be a wheeled quadratic operad as in Definition 5.1.3. Then the operadic part of its wheeled quadratic dual equals the ordinary quadratic dual of its operadic part

$$
\left(\mathcal{P}^{!}\right)_{o} \cong\left(\mathcal{P}_{o}\right)^{!}
$$

we denote it simply $\mathcal{P}_{o}^{!}$. The wheeled part of $\mathcal{P}^{!}$is the quotient of the wheeled part of the wheeled completion of $\mathcal{P}_{o}^{!}$,

$$
\left(\mathcal{P}^{!}\right)_{w} \cong\left(\left(\mathcal{P}_{o}^{!}\right)^{\circlearrowright}\right)_{w} / I_{w}^{\perp}
$$

by the ideal $I_{w}^{\perp}$ generated by $R_{w}^{\perp}$.

Proof. The double coset theorem gives isomorphisms

$$
P^{!}=\mathrm{F}^{\circlearrowright}\left\langle E^{\vee}\right\rangle /\left(R_{o}^{\perp}, R_{w}^{\perp}\right) \cong\left(\mathrm{F}^{\circlearrowright}\left\langle E^{\vee}\right\rangle /\left(R_{o}^{\perp}\right)\right) /\left(R_{w}^{\perp}\right) \cong\left(\mathcal{P}_{o}^{!}\right)^{\circlearrowright} / I_{w}^{\perp}
$$

Since the ideal $I_{w}^{\perp}$ consists of elements of biarities $(0, n), n \geq 1$, it does change the operadic part of the rightmost quotient. Isomorphisms (32) and (33) are then obtained by singling out the operadic and wheeled parts of the above display.

Let us see what happens if we apply Proposition 5.2.2 to the wheeled completion of an ordinary quadratic operad $\mathcal{P}$ given by the quotient (28). As we observed in Example 5.1.4, $\mathcal{P} \circlearrowright$ is a wheeled quadratic operad with $R_{o}=R$ and $R_{w}=0$, therefore, in (31), $R_{o}^{\perp}=R^{\perp}$ and $R_{w}^{\perp}=\mathrm{F}^{\circlearrowright}\left\langle E^{\vee}\right\rangle(0,1)$. By $(\underline{32}),\left(\mathcal{P}^{\circlearrowright}\right)_{0}^{!} \cong \mathcal{P}^{!}$, while (33) gives

$$
\left(\left(\mathcal{P}^{\circlearrowright}\right)^{!}\right)_{w} \cong\left(\left(\mathcal{P}^{!}\right)^{\circlearrowright}\right)_{w} / I_{w}^{\perp}
$$

with $I_{w}^{\perp}$ generated by $\mathrm{F}^{\circlearrowright}\left\langle E^{\vee}\right\rangle(0,1)$, that is, by relations represented by the decorated graphs:

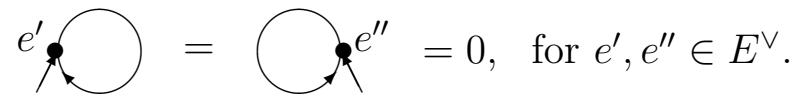

In the following examples we analyze in detail the wheeled quadratic duals of wheeled completions of some quadratic operads. As we saw above, this analysis necessarily involves the ordinary quadratic duals of these operads for which we refer to [19, Section II.3.2] or to the original source 9 . 
5.2.3. Example. Let us describe, using (34), the wheeled part of the wheeled quadratic dual $\left(\text { Ass }^{\circlearrowright}\right)^{!}$of the wheeled completion of the operad Ass. Since Ass ${ }^{!}=$Ass, the first step is to understand the wheeled part $\left(\text { Ass }^{\circlearrowright}\right)_{w}$ of the wheeled completion of Ass. It is easy to see that $\left(\text { Ass }^{\circlearrowright}\right)_{w}(n)$ is, for $n \geq 1$, spanned by graphs

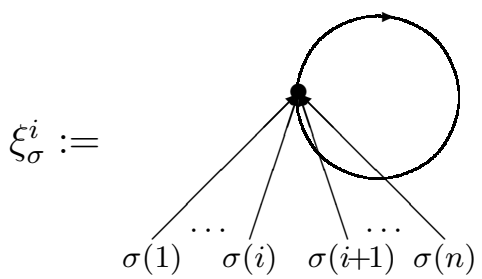

where $\sigma \in \Sigma_{n}$ and $0 \leq i \leq n$, such that both the 'left' group of inputs (labeled $\sigma(1), \ldots, \sigma(i)$ ) and the 'right' group of inputs (labeled $\sigma(i+1), \ldots, \sigma(n))$ is cyclically symmetric.

This cyclic symmetry can be seen as follows. By the associativity of the multiplication in Ass, one is allowed to single out the input edge labeled $\sigma(1)$, move it clockwise around the loop, and join it at the bottom of the left group. This operation generates a left action of the cyclic group $C_{i}:=\mathbb{Z} /(i)$ on the left group of labels. The cyclic symmetry of the right group can be explained similarly.

We claim that $\xi_{\sigma}^{i}=0$ modulo relations (35) whenever $i=n$ or $i=0$. Indeed, for $i=n$ one has, due to the associativity of the multiplication

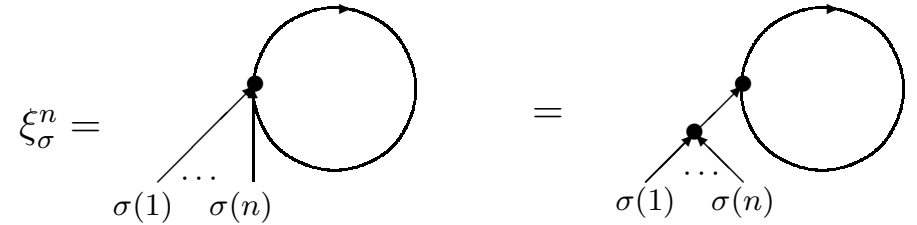

with the right element clearly belonging to the ideal generated by (35). By similar reasons, $\xi_{\sigma}^{1}$ is also zero modulo (35). We conclude that, for $n \geq 1$,

$$
\left(\mathrm{Ass}^{\circlearrowright}\right)_{w}^{!}(n) \cong \bigoplus_{0<i<n}\left(C_{i} \times C_{n-i}\right) \backslash \mathbf{k}\left[\Sigma_{n}\right]
$$

where $\left(C_{i} \times C_{n-i}\right) \backslash \mathbf{k}\left[\Sigma_{n}\right]$ is the left quotient of $\mathbf{k}\left[\Sigma_{n}\right]$ by the product of cyclic groups $C_{i} \times C_{n-i}$.

5.2.4. Example. Our next task is to describe the wheeled part of the wheeled quadratic dual $\left(\mathrm{Com}^{\circlearrowright}\right)$ ! of the wheeled completion of the operad Com. Since Com ${ }^{!}=$Lie, we need to analyze first the wheeled part $\left(\mathrm{Lie}^{\circlearrowright}\right)_{w}$ of the wheeled completion of Lie. The crucial fact is that the Jacoby identity

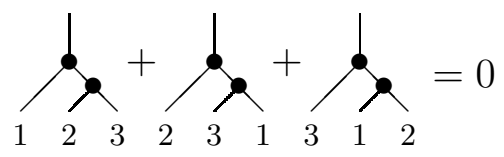


for the Lie bracket symbolized by $\boldsymbol{\alpha}$ implies that, in $\left(\mathrm{Lie}^{\circlearrowright}\right)_{w}(2)$,

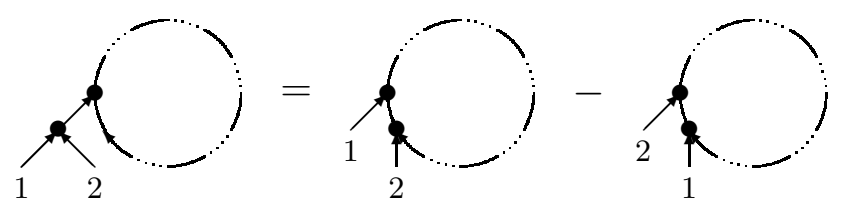

where the dotted oriented circle is the unique wheel of the underlying graph (which may or may not contain other vertices). It is not difficult to conclude from this that $\left(\mathrm{Lie}^{\circlearrowright}\right)_{w}(n)$ is, for $n \geq 1$, spanned by elements

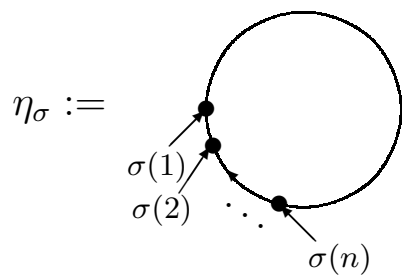

with $\sigma \in \Sigma_{n}$. It is also obvious that $\eta_{\sigma} \in I_{w}^{\perp}$ for $n=1$. So

$$
\left(\operatorname{Com}^{\circlearrowright}\right)_{w}^{!}(n) \cong \begin{cases}C_{n} \backslash \mathbf{k}\left[\Sigma_{n}\right], & \text { for } n \geq 2, \text { and } \\ 0, & \text { for } n=1,\end{cases}
$$

with the trivial action of $\Sigma_{n}$.

5.2.5. Example. In this example we describe the wheeled quadratic dual of the wheeled completion of Lie. Since $\mathrm{Lie}^{!}=\mathrm{Com}$, we need to start by investigating $\left(\mathrm{Com}^{\circlearrowright}\right)_{w}$. The graphs that represent elements of $\left(\mathrm{Com}^{\circlearrowright}\right)_{w}(n)$ are the same as in (36) $)$, but commutativity enables one to move all inputs of $\xi_{\sigma}^{i}$ to one side (say to the left) and arrange them into increasing order. Therefore $\left(\mathrm{Com}^{\circlearrowright}\right)_{w}(n)$ is, for $n \geq 1$, one dimensional, spanned by the directed graph

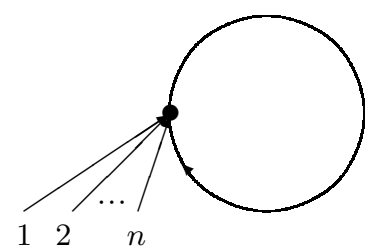

We argue as in Example 5.2.3 that, by associativity, the element represented by the above graph belongs to $I_{w}^{\perp}$ therefore, rather surprisingly,

$$
\left(\text { Lie }^{\circlearrowright}\right)_{w}^{!}(n)=0 \text { for } n \geq 1 \text {. }
$$

In other words, the wheeled part of $\left(\mathrm{Lie}^{\circlearrowright}\right) !$ is trivial.

Since each wheeled quadratic operad $\mathcal{P}$ is a particular case of an augmented properad, it makes sense to consider its bar construction introduced in Definition 4.2.1. Let us investigate how the 0-th homology $H_{0}\left(B^{\circlearrowright}(\mathcal{P}), \partial_{B}\right)$ is related to the wheeled quadratic dual of $\mathcal{P}$. It follows from simple combinatorics that the cooperad $B^{\circlearrowright}(\mathcal{P})=\mathrm{C}^{\circlearrowright}\left\langle\mathbf{w}^{-1} \overline{\mathcal{P}}\right\rangle$ is non-negatively graded and 
that an element $x \in B^{\circlearrowright}(\mathcal{P})$ with the underlying graph $G$ has degree 0 if and only if all vertices of $G$ are decorated by $\mathcal{P}(1,2)=E$. Therefore

$$
B^{\circlearrowright}(\mathcal{P})_{0} \cong \mathrm{C}^{\circlearrowright}\left\langle\mathbf{w}^{-1} E\right\rangle=\mathrm{C}^{\circlearrowright}\left\langle E \otimes \operatorname{sgn}_{2}\right\rangle .
$$

It is equally obvious that the degree 1 part $B^{\circlearrowright}(\mathcal{P})_{1}$ is spanned by elements whose underlying graphs have precisely one vertex decorated by $\mathcal{P}(1,3)$ and all remaining vertices decorated by $\mathcal{P}(1,2)=E$. The differential

$$
\partial_{B}: \mathrm{C}^{\circlearrowright}\left\langle E \otimes \operatorname{sgn}_{2}\right\rangle \rightarrow B^{\circlearrowright}(\mathcal{P})_{1}
$$

act as in (22), by contracting edges of the underlying graph. Let

$$
\mathcal{P}^{\mathrm{i}}:=H_{0}\left(B^{\circlearrowright}(\mathcal{P}), \partial_{B}\right)=\operatorname{Ker}\left(\partial_{B}: \mathrm{C}^{\circlearrowright}\langle E\rangle \rightarrow B^{\circlearrowright}(\mathcal{P})_{1}\right) .
$$

It is an exercise in linear algebra to prove that the cooperad $\mathcal{P}^{i}$ is precisely the linear dual the quadratic dual of $\mathcal{P}$, that is

$$
\mathcal{P}^{i \#}=\mathcal{P}^{!}
$$

5.2.6. Definition. A quadratic wheeled operad $\mathcal{P}$ is wheeled Koszul if the canonical inclusion of wheeled cooperads

$$
\iota:\left(\mathcal{P}^{\mathrm{i}}, \partial=0\right) \hookrightarrow\left(B^{\circlearrowright}(\mathcal{P}), \partial_{B}\right)
$$

is a homology isomorphism.

It follows from the above analysis that $\mathcal{P}$ is wheeled quadratic Koszul if and only if

$$
H_{>0}\left(B^{\circlearrowright}(\mathcal{P}), \partial_{B}\right)=0
$$

Another equivalent formulation is given in the following proposition.

5.2.7. Proposition. A quadratic wheeled operad $\mathcal{P}$ is wheeled Koszul if and only if the natural projection

$$
p:\left(\Omega^{\circlearrowright}\left(\mathcal{P}^{\mathrm{i}}\right), \partial_{\Omega}\right) \rightarrow(\mathcal{P}, \partial=0)
$$

of wheeled $d g$ operads is a homology isomorphism.

Proof. Observe that the projection (39) coincides with the composition

$$
\left(\Omega^{\circlearrowright}\left(\mathcal{P}^{\mathrm{i}}\right), \partial_{\Omega}\right) \stackrel{\Omega^{\circlearrowright}(\iota)}{\longrightarrow}\left(\Omega^{\circlearrowright}\left(B^{\circlearrowright}(\mathcal{P}), \partial_{\Omega}\right) \stackrel{\pi}{\longrightarrow} \mathcal{P}\right.
$$

in which $\Omega^{\circlearrowright}(\iota)$ is induced by the inclusion (38) and $\pi$ is the canonical map of Theorem 4.2.5 which is a homology. If $\mathcal{P}$ is wheeled Koszul then, by definition, $\iota$ is a homology isomorphism of nonnegatively graded co-properads, hence $\Omega^{\circlearrowright}(\iota)$ is, by Proposition 4.2.4, a homology isomorphism, too. The projection $p$ in (39) is then a composition of homology isomorphisms so it is also a homology isomorphism. 
The opposite implication follows from a similar analysis of the composition

$$
\left(\mathcal{P}^{\mathrm{i}}, \partial=0\right) \stackrel{c}{\rightarrow}\left(B^{\circlearrowright}\left(\Omega^{\circlearrowright}(\mathcal{P})\right), \partial_{B}\right) \stackrel{B^{\circlearrowright}(p)}{\longrightarrow}\left(B^{\circlearrowright}(\mathcal{P}), \partial_{B}\right)
$$

The importance of Proposition 5.2.7 is that (39) provides a functorial minimal resolution of wheeled quadratic Koszul operads in the category of wheeled operads. It is easy to verify that the left adjoint

$$
\text { 个: Proper } \rightarrow \text { PROP }
$$

to the forgetful functor PROP ${ }^{\circlearrowright} \rightarrow$ Proper $^{\circlearrowright}$, given by disjoint unions of the underlying graphs, it is an exact functor. Therefore ${ }^{\wedge}$ applied to (39) gives a functorial minimal resolution

$$
\widehat{p}:\left(\widehat{\Omega}^{\circlearrowright}\left(\mathcal{P}^{\mathrm{i}}\right), \partial_{\widehat{\Omega}}\right) \rightarrow(\widehat{\mathcal{P}}, \partial=0)
$$

of the wheeled PROP generated by $\mathcal{P}$ in the category of wheeled PROPs. It is clear that

$$
\left(\widehat{\Omega}^{\circlearrowright}\left(\mathcal{P}^{\mathrm{i}}\right), \partial_{\widehat{\Omega}}\right)=\left(\Gamma^{\circlearrowright}\left\langle\mathbf{w} \mathcal{P}^{\mathrm{i}}\right\rangle, \partial_{\widehat{\Omega}}\right)
$$

with $\partial_{\widehat{\Omega}}$ given on generators by the same formula as the cobar differential $\partial_{\Omega}$. Notice, however, that $\left(\widehat{\Omega}^{\circlearrowright}\left(\mathcal{P}^{\mathrm{i}}\right), \partial_{\widehat{\Omega}}\right)$ is not a cobar construction of $\mathcal{P}^{\mathrm{i}}$ in the category of wheeled PROPs.

5.2.8. Proposition. A wheeled quadratic operad $\mathcal{P}$ is wheeled Koszul if and only if $\mathcal{P} !$ is wheeled Koszul. The operadic part $\mathcal{P}_{o}$ of a wheeled Koszul operad $\mathcal{P}$ is Koszul in the ordinary sense.

Proof. The first statement is based on the isomorphism of the linear duals

$$
\left(B^{\circlearrowright}(\mathcal{P}), \partial_{B}\right)^{\#} \cong\left(\Omega^{\circlearrowright}\left(\mathcal{P}^{\#}\right), \partial_{\Omega}\right)
$$

and on the fact that, under the above isomorphism, the linear dual of the inclusion

$$
\iota:\left(\mathcal{P}^{\mathrm{i}}, \partial=0\right) \hookrightarrow\left(B^{\circlearrowright}(\mathcal{P}), \partial_{B}\right)
$$

is the projection

$$
p:\left(\Omega^{\circlearrowright}(\mathcal{P}), \partial_{\Omega}\right) \rightarrow\left(\mathcal{P}^{!}, \partial=0\right) .
$$

Since the linear dual is an exact functor, the Koszulity of $\mathcal{P}$ implies, by Proposition 5.2.7, the Koszulity of $\mathcal{P}$ !. The opposite implication follows from the above statement applied to $\mathcal{P}^{\text {! }}$ and the involution property $\left(\mathcal{P}^{!}\right)^{!} \cong \mathcal{P}$.

To prove the second part of the proposition, one needs to observe that the operadic part of the map (38) is the canonical inclusion

$$
\iota_{o}:\left(\mathcal{P}_{o}^{\mathrm{i}}, \partial=0\right) \hookrightarrow\left(B\left(\mathcal{P}_{o}\right), \partial_{B}\right),
$$

of the co-operad $\mathcal{P}_{o}^{\mathrm{i}}$ into its ordinary bar construction $\left(B\left(\mathcal{P}_{o}\right), \partial_{B}\right)$, and then invoke the definition of Koszulness in the form given in [7, Definition 2.23]. 
5.2.9. Example. Wheeled operads $\mathrm{Com}^{\circlearrowright}$, Ass ${ }^{\circlearrowright}$ and $\mathrm{Lie}^{\circlearrowright}$ are wheeled quadratic Koszul. Indeed, wheeled Koszulness of $\mathrm{Com}^{\circlearrowright}$ is Theorem 7.1.2 of Section 7 . The Koszulity of Ass ${ }^{\circlearrowright}$ follows from comparing the minimal model of Ass ${ }_{\infty}$ described in Theorem A to the bar construction of (Ass) given in Example 5.2.3. Finally, wheeled Koszulness of Lie was proved in [24].

We do not know whether there are (ordinary) quadratic Koszul operads whose wheeled completion is not wheeled Koszul.

5.2.10. Definition. Let $\mathcal{P}$ be an ordinary quadratic Koszul operad. We say that $\mathcal{P}$ is stably Koszul if the completion $\left(\Omega\left(\mathcal{P}^{!}\right), \partial_{\Omega}\right)^{\circlearrowright} \rightarrow\left(\mathcal{P}^{\circlearrowright}, \partial=0\right)$ of the canonical homology isomorphism $\left(\Omega\left(\mathcal{P}^{!}\right), \partial_{\Omega}\right) \rightarrow(\mathcal{P}, \partial=0)$ is a homology isomorphism, too.

Equivalently, a quadratic Koszul operad $\mathcal{P}$ is stably Koszul if the wheeled completion $\mathcal{P}_{\infty}^{\circlearrowright}$ of its minimal model $\mathcal{P}_{\infty}$ is a minimal model of the wheeled completion $\mathcal{P}$. The proof of the following proposition is a simple exercise.

5.2.11. Proposition. Let $\mathcal{P}$ be a quadratic operad such that $\mathcal{P} \circlearrowright$ is wheeled Koszul. Then $\mathcal{P}$ is stably Koszul if and only if $\left(\mathcal{P}^{\circlearrowright}\right) !=0$.

Proposition 5.2.11 together with Example 5.2.9 and Examples 5.2.3 5.2.5 imply that the operad Lie is stably Koszul while the operads Ass and Com are not.

\section{WheEled RESOlution of Ass AND its APpliCATiOns}

6.1. Wheeled completions of Ass and $\mathrm{Ass}_{\infty}$. As the natural functor Proper ${ }^{\circlearrowright} \rightarrow \mathrm{PROP}^{\circlearrowright}$ is exact, it is enough to understand homotopy type of wheeled completions of Ass and Ass $\infty$ in the category of wheeled properads. The generalization of all our results in this section to wheeled PROPs is immediate. Thus we work from now in the categories Proper and Proper ${ }^{0}$.

The operad, Ass, of associative algebras is defined as the quotient,

$$
\text { Ass }=\Gamma_{o p}\langle A\rangle /(R) .
$$

of the free operad, $\Gamma_{o p}\langle A\rangle$, generated by an $\Sigma$-module $A=\{A(n)\}$,

$$
A(n):= \begin{cases}k\left[\Sigma_{2}\right]=\operatorname{span}(\underbrace{}_{\sigma(1)})_{\sigma(2)} & \text { for } n=2 \\ 0 & \text { otherwise, }\end{cases}
$$

modulo the ideal generated by relations,

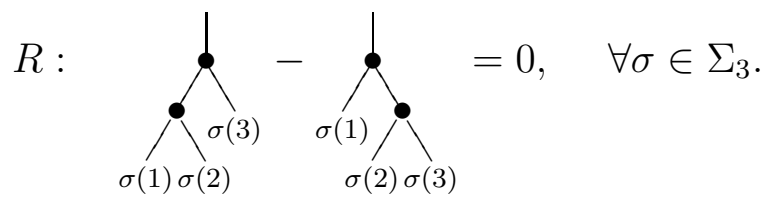


It is well-known [26] that the minimal resolution of Ass in the category of ordinary properads is the dg free properad, $\operatorname{Ass}_{\infty}:=\Gamma_{o p}\langle E\rangle$, generated by the $\Sigma$-bimodule $E=\{E(m, n)\}$,

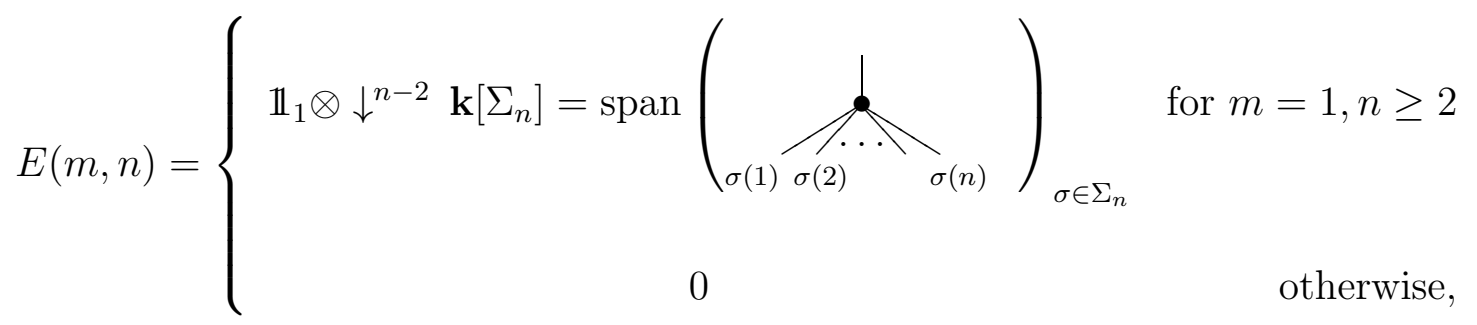

and equipped with the differential given on the generators as

$$
\partial \bigwedge_{\sigma(1)}=\sum_{k=0}^{n-2} \sum_{l=2}^{n-k}(-1)^{k+l(n-k-l)+1}
$$

A natural morphism of dg properads,

$$
p:\left(\text { Ass }_{\infty}, \partial\right) \longrightarrow(\text { Ass, } 0)
$$

given on generators by

$$
p\left(\underset{\sigma(1)}{\downarrow_{\sigma(2)}}\right):= \begin{cases}\text { Id } & \text { for } n=2 \\ 0 & \text { otherwise }\end{cases}
$$

is a quasi-isomorphism. The morphism $p$ induces an associated morphism of $\mathrm{dg}$ wheeled properads,

$$
p^{\circlearrowright}:\left(\left(\text { Ass }_{\infty}\right)^{\circlearrowright}, \partial\right) \longrightarrow\left(\text { Ass }^{\circlearrowright}, 0\right),
$$

which, however, can not be a quasi-isomorphism. Indeed [24], while

$$
p\left(\bigcap_{2}\right)=0
$$

the definition of $\partial$ implies

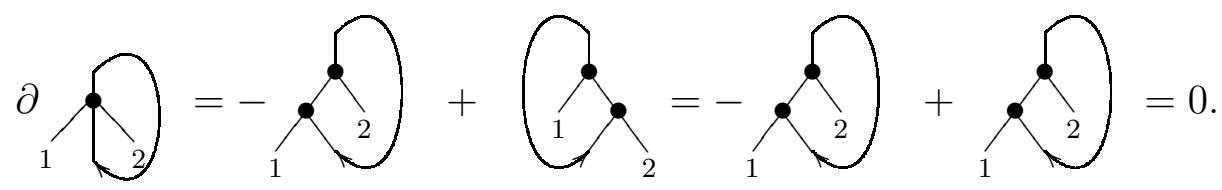

We therefore have non-trivial cohomology classes,

$$
\overbrace{\sigma(1)}:=[\overbrace{\sigma(2)} \sigma \in \Sigma_{2}
$$


in $H^{-1}\left(\left(\text { Ass }_{\infty}\right)^{\circlearrowright}, \partial\right)$ belonging to the kernel of $H^{*}\left(p^{\circlearrowright}\right)$.

6.1.1. Theorem. The cohomology group $H^{\bullet}\left(\left(\text { Ass }_{\infty}\right)^{\circlearrowright}, \partial\right)$ is concentrated in degrees 0 and -1 . Moreover,

$$
H^{0}\left(\left(\operatorname{Ass}_{\infty}\right)^{\circlearrowright}, \partial\right)=\text { Ass }^{\circlearrowright}
$$

and, as $\Sigma$-bimodules,

$$
H^{-1}\left(\left(\text { Ass }_{\infty}\right)^{\circlearrowright}, \partial\right)(m, n)= \begin{cases}\bigoplus_{p=1}^{n-1} k\left[\Sigma_{n}\right]^{C_{p} \times C_{n-p}} & \text { for } m=0, n \geq 2 \\ 0 & \text { otherwise }\end{cases}
$$

where $C_{p} \times C_{n-p}$ is the subgroup of $\Sigma_{n}$ generated by two commuting cyclic permutations $(12 \ldots p)$ and $(p+1 \ldots n)$, and $k\left[\Sigma_{n}\right]^{C_{p} \times C_{n-p}}$ stands for coinvariants.

Proof. The space $\left(\mathrm{Ass}_{\infty}\right)^{\circlearrowright}$ is spanned by graphs of genus 0 and 1 , and the differential $\partial$ preserves the associated genus decomposition,

$$
\left(\operatorname{Ass}_{\infty}\right)^{\circlearrowright}=\mathfrak{A}^{\uparrow} \oplus \mathfrak{A}^{\circlearrowright} .
$$

The subcomplex $\left(\mathfrak{A}^{\uparrow}, \partial\right)$ is spanned, by definition, by graphs of genus zero and hence is isomorphic to $\left(\operatorname{Ass}_{\infty}, \partial\right)$ so that $H\left(\mathfrak{A}^{\uparrow}, \partial\right)=$ Ass. Thus the main job is to compute the cohomology, $H\left(\mathfrak{A}^{\circlearrowright}, \partial\right)$, of the subcomplex $\mathfrak{A}^{\circlearrowright}$ spanned by graphs of genus one, i.e. by graphs of the form

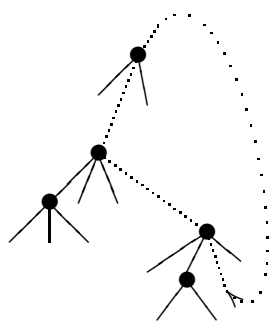

with internal edges lying in the wheel dotted.

The following terminology will be useful: the vertices of an element $G \in \mathfrak{A}^{\circlearrowright}$ which lie on the wheel are called cyclic. For example, the graph shown above has three cyclic vertices and two noncyclic vertices.

It is clear that

$$
F_{p} \mathfrak{A}^{\circlearrowright}:=\operatorname{span}\left\{f \in \mathfrak{A}^{\circlearrowright}: \text { total number of cyclic vertices } \geq p\right\},
$$

defines a filtration in the complex $\left(\mathfrak{A}^{\circlearrowright}, \partial\right)$. Let $\left\{\mathfrak{A}_{p}^{\circlearrowright}, \partial_{p}\right\}_{p \geq 0}$ be the associated spectral sequence.

Step 1. Our first target is to compute the cohomology, $\mathfrak{A}_{1}^{\circlearrowright}$, of the zeroth term, $\left\{\mathfrak{A}_{0}^{\circlearrowright}, \partial_{0}\right\}$, of this spectral sequence. Consider the filtration,

$$
\mathcal{F}_{p} \mathfrak{A}_{0}^{\circlearrowright}:=\operatorname{span}\left\{G \in \mathfrak{A}_{0}^{\circlearrowright}: \begin{array}{l}
\text { total number of internal edges and legs } \\
\text { attached to cyclic vertices of } G
\end{array} \leq p\right\},
$$

and let $\left\{\mathcal{E}_{r} \mathfrak{A}_{0}^{\circlearrowright}, \delta_{r}\right\}_{r \geq 0}$ be the associated spectral sequence. We shall show below that the latter degenerates at the second term so that $\mathcal{E}_{2} \mathfrak{A}_{0}^{\circlearrowright}=H\left(\mathfrak{A}_{0}^{\circlearrowright}, \partial_{0}\right)=\mathfrak{A}_{1}^{\circlearrowright}$.

The differential $\delta_{0}$ in $\mathcal{E}_{0} \mathfrak{A}_{0}^{\circlearrowright}$ is given by its values on the vertices as follows: 
(i) on every noncyclic vertex one has $\delta_{0}=\partial$, the differential in Ass $_{\infty}$;

(ii) on every cyclic vertex $\delta_{0}=0$.

Hence, modulo the action of finite groups, the complex $\left(\mathcal{E}_{0} \mathfrak{A}_{0}^{\circlearrowright}, \delta_{0}\right)$ is isomorphic to the direct sum of tensor products of copies of the complex $\left(\operatorname{Ass}_{\infty}, \partial\right)$. By Künneth's and Mashke's theorems, we get,

$$
\mathcal{E}_{1} \mathfrak{A}_{0}^{\circlearrowright}=V_{1} / h\left(V_{2}\right)
$$

where

- $V_{1}$ is the subspace of $\mathfrak{A}^{\circlearrowright}$ consisting of all those graphs whose every noncyclic vertex is $\not$;

- $V_{2}$ is the subspace of $\mathfrak{A}^{\circlearrowright}$ whose every noncyclic vertex is either $\boldsymbol{k}$ or $\boldsymbol{k}$ with the number of vertices of the latter type $\geq 1$;

- the map $h: V_{2} \rightarrow V_{1}$ is $\Sigma$-equivariant and is given on noncyclic vertices by

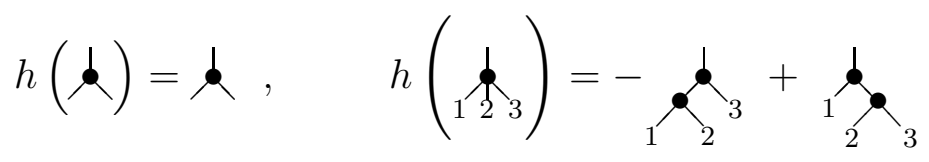

and on all cyclic vertices $h$ is set to be the identity.

A representative of a typical element in $\mathcal{E}_{1} \mathfrak{A}^{\circlearrowright}$ looks as

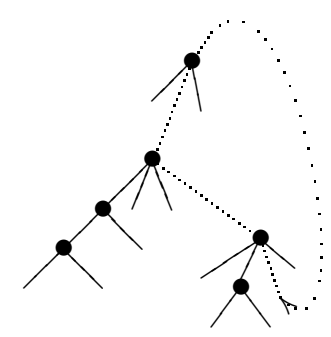

The differential $\delta_{1}$ in $\mathcal{E}_{1} \mathfrak{A}^{\circlearrowright}$ is given by its values on vertices as

(i) on every noncyclic vertex one has $\delta_{1}=0$;

(ii) on every cyclic vertex one has

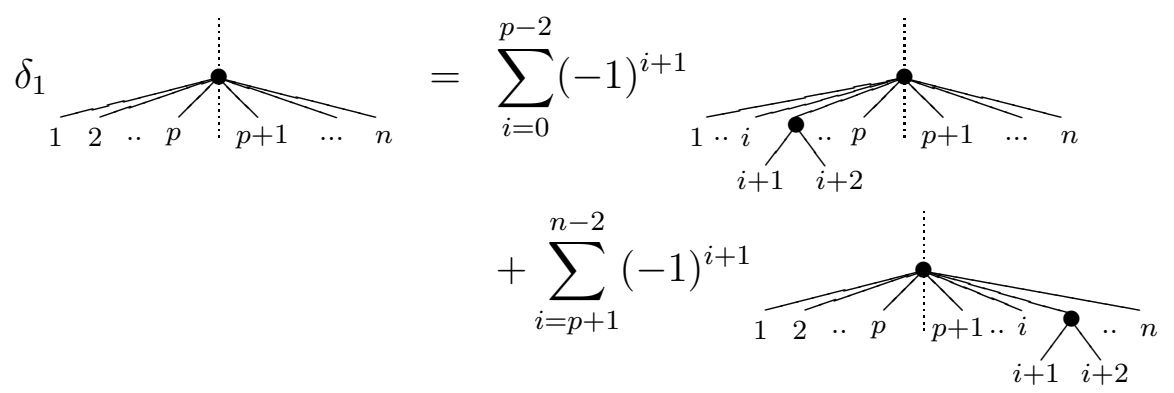

To compute $\mathcal{E}_{2} \mathfrak{A}^{\circlearrowright}=H\left(\mathcal{E}_{1} \mathfrak{A}^{\circlearrowright}, \delta_{1}\right)$ let us return back to the well-known complex Ass ${ }_{\infty}$ : the data $F_{p} \mathrm{Ass}_{\infty}:=\operatorname{span}\left\{G \in \mathrm{Ass}_{\infty}:\right.$ number of edges attached to the root vertex of $\left.G \leq p\right\}$ 
is clearly a filtration of the complex $\left(\right.$ Ass $\left._{\infty}, \delta\right)$ whose spectral sequence, $\left\{E_{r} \mathrm{Ass}_{\infty}, d_{r}\right\}_{r \geq 0}$, must converge to Ass. Its first term, $E_{1}$ Ass $_{\infty}=H\left(E_{0} \mathrm{Ass}_{\infty}, d_{0}\right)$ is spanned by trees whose root vertex may have any number of attached half-edges while all other vertices are binary, $\boldsymbol{\ell}$. The differential $d_{1}$ is non-trivial only on the root vertex on which it is given by,

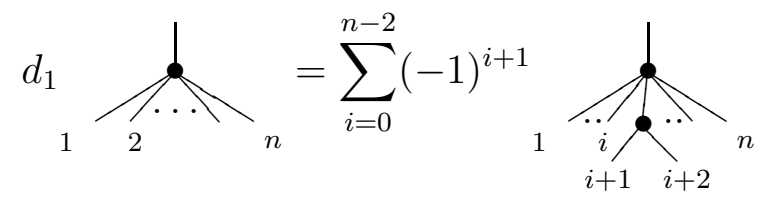

It is clear that this spectral sequence must degenerate at $E_{2} \mathrm{Ass}_{\infty}=H\left(E_{1} \mathrm{Ass}_{\infty}, d_{1}\right)$ implying the isomorphism $H\left(E_{1} \mathrm{Ass}_{\infty}, d_{1}\right)=$ Ass. Now let us modify the complex $\left(E_{1} \mathrm{Ass}_{\infty}, d_{1}\right)$ by adding to the space $E_{1}$ Ass $_{\infty}$ the trees whose root vertex is a degree -1 corolla $\phi$ while all other vertices are binary $\alpha$.

Denote this extension of $E_{1} \mathrm{Ass}_{\infty}$ by $E_{1}^{+} \mathrm{Ass}_{\infty}$, and define a differential $d_{1}^{+}$on $E_{1}^{+} \mathrm{Ass}_{\infty}$ be setting its values on the non-root vertices to be zero while on the root $(1, n)$-vertex as follows

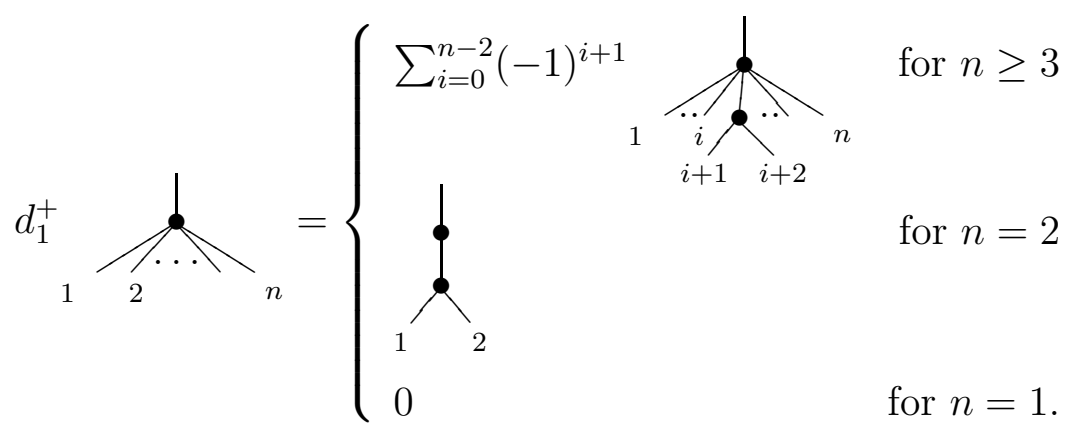

Claim. The cohomology of the complex $\left(E_{1}^{+} \mathrm{Ass}_{\infty}, d_{1}^{+}\right)$is a one dimensional vector space spanned by $\downarrow$.

Proof of the claim. Consider a 2-step filtration, $F_{0} \subset F_{1}$ of the complex $\left(E_{1}^{+} \mathrm{Ass}_{\infty}, d_{1}^{+}\right)$by the number of $\boldsymbol{\phi}$. The zero-th term of the associated spectral sequence is isomorphic to the direct sum of the complexes,

$$
\left(E_{1} \mathrm{Ass}_{\infty}, d_{1}\right) \oplus\left(\downarrow E_{1} \mathrm{Ass}_{\infty}, d_{1}\right) \oplus(\operatorname{span}\langle\boldsymbol{\varphi}\rangle, 0)
$$

so that the next term of the spectral sequence is

$$
\text { Ass } \oplus \downarrow \text { Ass } \oplus\langle\phi\rangle
$$

with the differential being zero on $\downarrow$ Ass $\oplus\langle\varphi\rangle$ and the natural isomorphism,

$$
\downarrow: \text { Ass } \longrightarrow \downarrow \text { Ass }
$$

on the remaining summand. Hence the claim follows.

Comparing differentials (42) and (43), we see that, modulo actions of finite groups, the complex $\left(\mathcal{E}_{1} \mathfrak{A}_{0}^{\circlearrowright}, \delta_{1}\right)$ is isomorphic to the tensor product of a trivial complex (i.e. one with zero differential) with the tensor powers of the complex, $\left(E_{1}^{+} A s_{\infty}, d_{1}^{+}\right)$. Then the above Claim implies that 
$\mathcal{E}_{2} \mathfrak{A}_{0}^{\circlearrowright}=H\left(\mathcal{E}_{1} \mathfrak{A}_{0}^{\circlearrowright}, \delta_{1}\right)$ is spanned by the wheeled graphs whose every vertex is cyclic and is either binary or ternary, i.e. by graphs of the form,

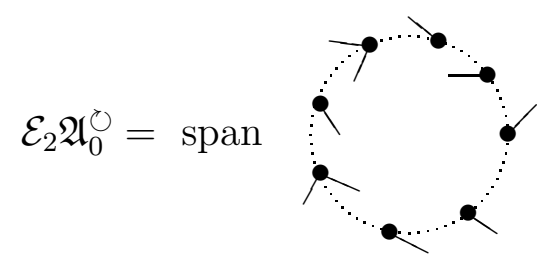

with legs numbered by integers (not shown). The induced differential, $\delta_{2}$, on such graphs is obviously zero, so that the spectral sequence $\left(\mathcal{E}_{p} \mathfrak{A}_{0}^{\circlearrowright}, \delta_{p}\right)$ degenerates giving us an isomorphism $\mathfrak{A}_{1}^{\circlearrowright}=\lim _{p \rightarrow \infty}\left(\mathcal{E}_{p} \mathfrak{A}_{0}^{\circlearrowright}, \delta_{p}\right) \simeq \mathcal{E}_{2} \mathfrak{A}_{0}^{\circlearrowright}$.

Step 2. We have shown above that the first term, $\mathfrak{A}_{1}^{\circlearrowright}$, of the spectral sequence $\left\{\mathfrak{A}_{p}^{\circlearrowright}, \partial_{p}\right\}_{p \geq 0}$ can be identified with the vector space spanned by wheeled graphs of the form (44). The induced differential $\partial_{1}$ on $\mathfrak{A}_{1}^{\circlearrowright}$ can then be described on generators as follows:

$$
\partial_{1}:=0, \quad \partial_{1}=0, \quad \partial_{1}=\vdots_{\vdots}=0
$$

Now the theorem follows immediately from the following

Claim. The spectral sequence $\left\{\mathfrak{A}_{p}^{\circlearrowright}, \partial_{p}\right\}_{p \geq 0}$ degenerates at $p=2$ with an isomorphism of $\Sigma$ bimodules,

$$
\mathfrak{A}_{2}^{\circlearrowright}(0, n)=H\left(\mathfrak{A}_{1}^{\circlearrowright}, \partial_{1}\right)(0, n)= \begin{cases}\operatorname{Ass}^{\circlearrowright}(0,1) & \text { for } n=1 \\ \operatorname{Ass}^{\circlearrowright}(0, n) \oplus \bigoplus_{p=1}^{n-1} \downarrow k\left[\Sigma_{n}\right]^{C_{p} \times C_{n-p}} & \text { for } n \geq 2 .\end{cases}
$$

Proof of the Claim. Let $\left({ }_{r} \mathfrak{A}_{s}^{\circlearrowright}, \partial_{1}\right)$ be, for $r+s=n$, the subcomplex of $\left(\mathfrak{A}_{1}^{\circlearrowright}, \partial_{1}\right)(0, n)$ spanned by graphs whose legs entering the cycle from the left have labels $1, \ldots, r$ and legs merging from the right labels $r+1, \ldots, n$. Clearly each ${ }_{r} \mathfrak{A}_{s}^{\circlearrowright}$ is a $\left(\Sigma_{r} \times \Sigma_{s}\right)$-module and the $\Sigma_{n}$-space $\mathfrak{A}_{1}^{\circlearrowright}$ is the sum of induced representations

$$
\mathfrak{A}_{1}^{\circlearrowright} \cong \bigoplus_{r+s=n} \operatorname{Ind}_{\Sigma_{r} \times \Sigma_{s}}^{\Sigma_{n}} \mathfrak{A}_{s}^{\circlearrowright}
$$

Our proof of the claim will be based on showing that the cohomology of $\left({ }_{r} \mathfrak{A}_{s}^{\circlearrowright}, \partial_{1}\right)$ is isomorphic to the (regraded) cohomology of the moduli space of configurations ${ }_{r} U_{s}$ defined in the following paragraph.

First, let ${ }_{r} \widetilde{M}_{s}$ be the space of configurations of two types of labeled, not necessarily distinct, points on the unit cycle $S^{1}$ - "left" points labeled by $1, \ldots, r$ and "right" points labeled $r+1, \ldots, n$. Let ${ }_{r} \widetilde{U}_{s}$ be the open subspace of configurations such that points of the same type do not collide, that is, only "left-right" collisions are allowed in ${ }_{r} \widetilde{U}_{s}$. We denote by ${ }_{r} M_{s}$ and ${ }_{r} U_{s}$ the corresponding moduli spaces, ${ }_{r} M_{s}:={ }_{r} \widetilde{M}_{s} / S^{1}$ and ${ }_{r} U_{s}:={ }_{r} \widetilde{U}_{s} / S^{1}$. Let us prove that

$$
H^{\bullet}\left({ }_{r} \mathfrak{A}_{s}^{\circlearrowright}, \partial_{1}\right) \cong H^{-\bullet}\left({ }_{r} U_{s}\right)
$$


To this end, consider the complement ${ }_{r} N_{s}:={ }_{r} M_{s} \backslash{ }_{r} U_{s}$. It is clear that ${ }_{r} M_{s}$ is a compact orientable $(r+s-1)$-dimensional manifold and ${ }_{r} N_{s}$ its closed subspace. By definition, ${ }_{r} N_{s}$ consists of equivalence classes of configurations such that two (or more) points of the same type coincide. It therefore looks locally as an intersection of hyperplanes, thus it is a strong deformation retract of some open neighborhood ${ }_{r} O_{s} \supset{ }_{r} N_{s}$. Denote finally ${ }_{r} K_{s}:={ }_{r} M_{s} \backslash{ }_{r} O_{s}$. By [10, Proposition 3.46],

$$
H^{-\bullet}\left({ }_{r} K_{s}\right) \cong H_{r+s-1+\bullet}\left({ }_{r} M_{s},{ }_{r} O_{s}\right),
$$

where, since ${ }_{r} N_{s}$ is a deformation retract of ${ }_{r} O_{s}, H_{\bullet}\left({ }_{r} M_{s},{ }_{r} O_{s}\right) \cong H_{\bullet}\left({ }_{r} M_{s},{ }_{r} N_{s}\right)$ and, similarly, $H^{\bullet}\left({ }_{r} K_{s}\right) \cong H^{\bullet}\left({ }_{r} U_{s}\right)$. We see that

$$
H^{-\bullet}\left({ }_{r} U_{s}\right) \cong H_{r+s-1+\bullet}\left({ }_{r} M_{s},{ }_{r} N_{s}\right)
$$

To describe the right hand side of (47), notice that ${ }_{r} M_{s}$ has a cell structure, with codimension $d$ cells corresponding to types of configurations with exactly $d$ collisions. The closed subspace ${ }_{r} N_{s} \subset{ }_{r} M_{s}$ is a cell subcomplex and codimension $d$ cells of the relative cellular chain complex $C\left({ }_{r} M_{s},{ }_{r} N_{s}\right)$ correspond to types of configurations with exactly $d$ left-right collisions. Obviously, these types are parametrized by graphs (44) with $d$ triple points. One easily sees that $\left(C\left({ }_{r} M_{s},{ }_{r} N_{s}\right), \partial\right)$ is isomorphic to $\left({ }_{r} \mathfrak{A}_{s}^{\circlearrowright}, \partial_{1}\right)$ with the opposite grading shifted by $r+s-1$, giving rise to the isomorphism

$$
H_{r+s-1+\bullet}\left({ }_{r} M_{s},{ }_{r} N_{s}\right) \cong H_{r+s-1+\bullet}\left(C\left({ }_{r} M_{s},{ }_{r} N_{s}\right), \partial\right) \cong H^{\bullet}\left({ }_{r} \mathfrak{A}_{s}^{\circlearrowright}, \partial_{1}\right)
$$

which, combined with (47), gives (46).

On the other hand, the homotopy type of ${ }_{r} \widetilde{U}_{s}$ is easy to describe: there exists an equivariant deformation retraction that distributes left points evenly around the cycle so that two adjacent points are precisely $2 \pi / r$ apart, leaving the point labeled by 1 unchanged, and similarly distributes right points leaving the one labeled $r+1$ fixed. The configurations obtained in this way are parametrized by the position of points labeled 1 and $p+1$, plus the cyclic orders of left and right points, that is

$$
{ }_{r} \widetilde{U}_{s} \sim\left(C_{r} \times C_{s}\right) \backslash\left(\Sigma_{r} \times \Sigma_{s}\right) \times S_{r}^{1} \times S_{s}^{1}
$$

where $S_{i}^{1}:=S^{1}$ if $i \geq 1$ while $S_{0}^{1}:=$ the point. By the definition of ${ }_{r} U_{s}$,

$$
{ }_{r} U_{s} \sim\left(C_{r} \times C_{s}\right) \backslash\left(\Sigma_{r} \times \Sigma_{s}\right) \times{ }_{r} S_{s}^{1},
$$

where ${ }_{r} S_{s}^{1}:=S^{1}$ if $r, s \geq 1$ and ${ }_{r} S_{s}^{1}:=$ the point if $(r, s) \in\{(1,0),(0,1)\}$.

Equation (46) implies

$$
H^{\bullet}\left({ }_{r} \mathfrak{A}_{s}^{\circlearrowright}, \partial_{1}\right) \cong H^{-\bullet}\left({ }_{r} U_{s}\right) \cong \mathbf{k}\left[\Sigma_{r} \times \Sigma_{s}\right]^{C_{r} \times C_{s}} \otimes H^{-\bullet}\left({ }_{r} S_{s}^{1}\right),
$$


which, combined with (45), leads to

$$
\begin{aligned}
H^{\bullet}\left(\mathfrak{A}_{1}^{\circlearrowright}, \partial_{1}\right)(0, n) & \cong \bigoplus_{r+s=n} \mathbf{k}\left[\Sigma_{n}\right]^{C_{r} \times C_{s}} \otimes H^{-\bullet}\left({ }_{r} S_{s}^{1}\right) \\
& =\left\{\begin{array}{ll}
\operatorname{Ass}^{\circlearrowright}(0,1) & \text { for } n=1 \\
\operatorname{Ass}^{\circlearrowright}(0, n) \oplus \bigoplus_{p=1}^{n-1} \downarrow k\left[\Sigma_{n}\right]^{C_{p} \times C_{n-p}} & \text { for } n \geq 2 .
\end{array} .\right.
\end{aligned}
$$

proving the claim.

6.1.2. Proposition. There is an isomorphism of graded wheeled properads,

$$
H^{\bullet}\left(\left(\text { Ass }_{\infty}\right)^{\circlearrowright}, \partial\right)=\mathrm{S}^{\circlearrowright}
$$

where $\mathrm{S}^{\circlearrowright}$ is the wheeled completion of the quotient properad,

$$
\mathrm{S}:=\Gamma\langle\hat{A}\rangle /(R),
$$

with the $\Sigma$-bimodule $\hat{A}=\{\hat{A}(m, n)\}_{m, n \geq 0}$ given by

$$
\hat{A}(m, n):= \begin{cases}\mathbb{1}_{1} \otimes k\left[\Sigma_{2}\right]=\operatorname{span}(\underbrace{}_{\sigma(1)})_{\sigma(2)} & \text { for } m=1, n=2 \\ \downarrow k\left[\Sigma_{2}\right]=\operatorname{span}(\underbrace{}_{\sigma(1)})_{\sigma(2)} & \text { for } m=0, n=2 \\ 0 & \text { otherwise, }\end{cases}
$$

and relations given by (40) and,
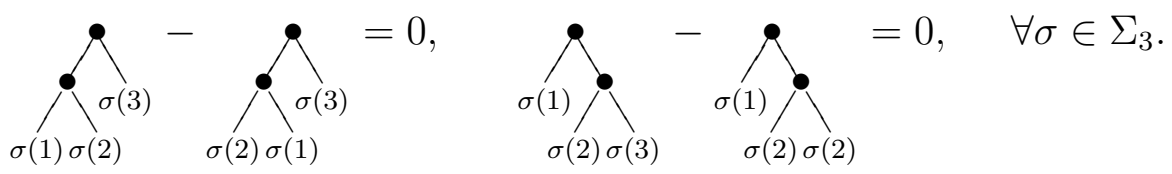

Proof. It was shown in the proof of Theorem 6.1.1 that every generator $e \in k\left[\Sigma_{n}\right]^{C_{p} \times C_{n-p}} \subset$ $H^{-1}\left(\left(\text { Ass }_{\infty}\right)^{\circlearrowright}\right)$ can be canonically identified with the $C_{p} \times C_{n-p^{-}}$orbit,

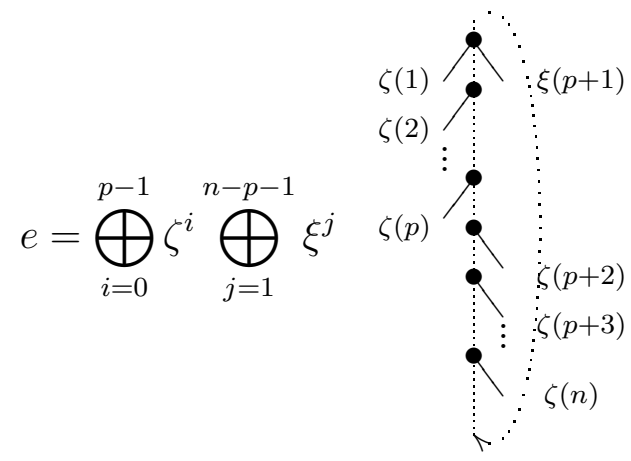


of a planar wheeled graph which has precisely one ternary vertex and $n-2$ binary vertices of which $p-1$ vertices have the non-cyclic input leg pointing "outside" the planar wheel. To prove the Proposition it is enough to show that every such a linear combination of graphs is homologically equivalent in the complex $\left(\left(\operatorname{Ass}_{\infty}\right)^{\circlearrowright}, \partial\right)$ to a uniquely defined element in $\mathbf{S}^{\circlearrowright}$. The latter can be easily established by induction on the number of vertices starting with the following initial step,

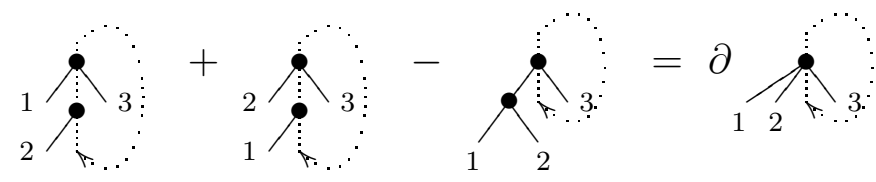

6.1.3. Remark. It is worth emphasizing a correspondence,

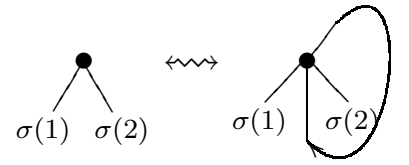

between the $(0,2)$ generators of the operad $\mathbf{S}$ and the wheeled elements in $\left(\text { Ass }_{\infty}\right)^{\circlearrowright}$.

6.2. Directed oriented ribbon graphs. The authors of [20] were able to compute cohomology of the directed, $\left(\mathfrak{G}_{g}^{\uparrow}(m, n), \delta\right)$, version (without wheels though) of Kontsevich's ribbon graph complex [11] consisting of oriented ribbon graphs of genus $g$ with $n$ input and $m$ output legs, and show that it is acyclic almost everywhere. Wheeled directed ribbon graphs, $\left(\mathfrak{G}_{g}^{\circlearrowright}(m, n), \delta\right)$, provide us with a finer approximation to the original Kontsevich's complex than $\left(\mathfrak{G}_{g}^{\uparrow}(m, n), \delta\right)$, and in this case the cohomology groups $H^{\bullet}\left(\mathfrak{G}_{g}^{\circlearrowright}(m, n), \delta\right)$ turn out to be non-trivial in many degrees.

Let us now give a precise description of all key actors. Consider first the vector space $\widehat{\mathfrak{G}}_{g}^{\circlearrowright}(m, n)$ spanned by directed oriented ribbon $(m, n)$-graph 3 which are triples, $(G$, or $(G)$, ribbon structure), where

- $G$ is a graph from $G^{\circlearrowright}(m, n)$ satisfying the conditions: (i) every vertex of $G$ has valence at least 3 (with at least one ingoing and at least one outgoing edges), (ii) vertices of any particular closed path in $G$ are purely "operadic", i.e. they all have either precisely one incoming edge or they all have precisely one outgoing edge, and (iii) the associated geometric realization $|G|$ has genus $g$;

- an orientation on a directed $(m, n)$-graph $G$ is, by definition, an orientation on the vector space, $\mathbb{R}^{\operatorname{vert}(G)} \oplus \mathbb{R}^{m} \oplus \mathbb{R}^{n}$ which is in fact the same as an orientation on $\mathbb{R}^{e(G)} \oplus H_{\bullet}(|G|, \mathbb{R})$, where $e(G)$ is the cardinality of the set, edge $(G)$, of internal edges of $G$, and $H_{\bullet}(|G|, \mathbb{R})$ is the homology of $|G|$ (see, e.g. [4]). Thus we can understand an orientation of $G$ as an element

$$
\operatorname{or}(G):=\operatorname{or}_{1}(G) \otimes \mathrm{or}_{2}(G) \in \operatorname{det} \mathbb{R}^{e(G)} \otimes \operatorname{det} H_{\bullet}(|G|, \mathbb{R}) .
$$

\footnotetext{
${ }^{3}$ The set, $\mathrm{G}^{\circlearrowright}(m, n)$, of directed $(m, n)$-graphs are defined in $\S 2$.
} 
- a ribbon structure on a directed $(m, n)$-graph $G$ is, by definition, an ordering of the set, in $(v)$, of incoming edges and the set, out $(v)$, of outgoing edges for each vertex $v \in \operatorname{vert}(G)$; it can be equivalently understood as a cyclic ordering of the set $i n(v) \cup \operatorname{out}(v)$.

We often abbreviate a triple from $\hat{\mathfrak{G}}_{g}^{\circlearrowright}$ by $(G$, or $(G))$ or even by $G$.

6.2.1. Definition. The quotient of the vector space $\hat{\mathfrak{G}}^{\circlearrowright}(m, n)$ by the equivalence relation

$$
(G,-o r(G))=-(G, \text { or }(G))
$$

is denoted by $\mathfrak{G}_{g}(m, n)$. It is naturally an $\mathbb{N}$-graded vector space,

$$
\mathfrak{G}_{g}^{\circlearrowright}(m, n)=\bigoplus_{n \geq 1} \mathfrak{G}_{g}^{n}(m, n)
$$

with respect to the number of vertices of its elements, i.e. $\mathfrak{G}_{g}^{n}(m, n):=\left\{G \in \mathfrak{G}_{g}^{\circlearrowright}(m, n)\right.$ : $|\operatorname{vert}(G)|=n\}$.

In fact, it is only the part $\mathfrak{G}_{g}:=\mathfrak{G}_{g}^{\circlearrowright}(0,0)$ which can be regarded as a directed version of Kontsevich's ribbon graph complex; by contrast to $\mathfrak{G}_{g}^{\uparrow}(0,0)=0$, the part $\mathfrak{G}_{g}^{\circlearrowright}(0,0)$ is highly non-trivial.

6.2.2. Fact $([11,20])$. The graded vector space $\mathfrak{G}_{g}^{\circlearrowright}(m, n)$ can be made into a cochain complex by setting

$$
\partial(G, \operatorname{or}(G)):=\sum_{G^{\prime} \mid G^{\prime} / e=G}\left(G^{\prime}, \operatorname{or}\left(G^{\prime}\right)\right)
$$

where the summation goes over all connected directed oriented ribbon $(m, n)$-graphs $G^{\prime}$ such that

- $G$ can be obtained from $G^{\prime}$ by contracting an internal edge $e \in e\left(G^{\prime}\right)$ which is not a loop;

- the cyclic ordering of edges at the vertex $v \in \operatorname{vert}(G)$ into which $e$ contracts agrees in the obvious sense with the one induced from the contraction (see, e.g., §2.2.2 in [4] for full details and pictures);

- $\operatorname{or}\left(G^{\prime}\right)=\left(\operatorname{or}_{1}(G) \wedge e\right) \otimes o r_{2}(G)$.

Set $\mathfrak{G}_{g}^{\circlearrowright}:=\left\{\mathfrak{G}_{g}^{\circlearrowright}(m, n)\right\}_{m, n \geq 0}$. It is naturally an $\Sigma$-bimodule.

6.2.3. Theorem. $H^{\bullet}\left(\mathfrak{G}_{g}^{\circlearrowright}\right)$ is isomorphic as an $\Sigma$-bimodule to the ordinary PROP, 4

$$
\frac{\mathrm{S}^{\circlearrowright} *\left(\mathrm{~S}^{\circlearrowright}\right)^{\dagger}}{I_{0}}
$$

where $\mathrm{S}^{\circlearrowright}$ is defined in $\S 6.1 .2, *$ stands for the free product of ordinary PROPs, and the ideal $I_{0}$ is generated by the elements

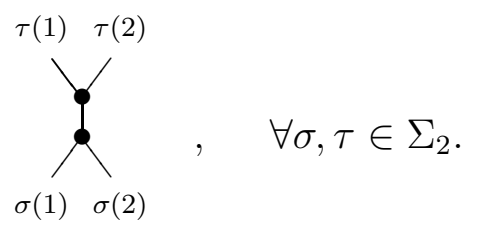

\footnotetext{
${ }^{4}$ For a PROP $P=\{P(m, n)\}$ we denote by $P^{\dagger}=\left\{P^{\dagger}(m, n)\right\}$ the associated PROP with "reversed flow", i.e. $P^{\dagger}(m, n):=P(n, m)$.
} 
Proof. Exactly the same argument as in the final section in [20] establishes an isomorphism of the complex $\left(\mathfrak{G}_{g}^{\circlearrowright}, \partial\right)$ with the operadic wheeled completion $\left[\right.$, $\left(\mathrm{IB}_{\infty}^{q \rightarrow}, \delta\right)$, of the minimal $\mathrm{dg}$ PROP resolution, $\left(\mathrm{IB}_{\infty}, \delta\right)$, of the PROP, IB, of infinitesimal associative bialgebras, which is, by definition, the quotient,

$$
\mathrm{IB}:=\frac{\operatorname{Ass} * \mathrm{Ass}^{\dagger}}{I}
$$

by the ideal $I$ generated by the elements

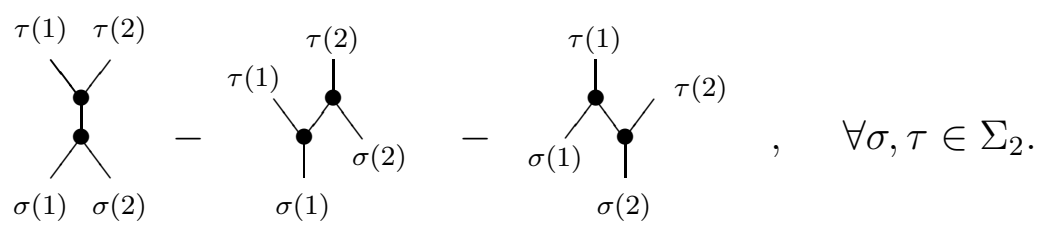

As the ideal $I$ is related to the ideal $I_{0}$ via distributive law (see [6, 20]), one can apply Theorem 3.11.1(ii) from [24] to compute the cohomology of the complex $\left(\mathrm{IB}_{\infty}^{9 \rightarrow}, \delta\right)$ as an $\Sigma$-bimodule,

$$
H^{\bullet}\left(\mathrm{IB}_{\infty}^{\hookrightarrow}, \delta\right) \simeq \frac{H^{\bullet}\left(\left(\mathrm{Ass}_{\infty}\right)^{\circlearrowright}\right) * H^{\bullet}\left(\left(\mathrm{Ass}_{\infty}\right)^{\circlearrowright}\right)^{\dagger}}{I_{0}}
$$

Finally, Proposition 6.1.2 completes the proof.

6.3. Proof of Theorem $\mathbf{C}$ (see $\S 1$ ). Since $H^{\bullet}\left(\mathfrak{G}_{g}\right)$ is, by definition of $\mathfrak{G}_{g}$, equal to the $H^{\bullet}\left(\mathfrak{G}_{g}^{\circlearrowright}\right)(0,0)$ part of the $\Sigma$-bimodule $H^{\bullet}\left(\mathfrak{G}_{g}^{\circlearrowright}\right)=\left\{H^{\bullet}\left(\mathfrak{G}_{g}^{\circlearrowright}\right)(m, n)\right\}_{m, n \geq 0}$, we have an isomorphism

$$
H^{\bullet}\left(\mathfrak{G}_{g}\right) \simeq \frac{H^{\bullet}\left(\left(\text { Ass }_{\infty}\right)^{\circlearrowright}\right) * H^{\bullet}\left(\left(\text { Ass }_{\infty}\right)^{\circlearrowright}\right)^{\dagger}}{I_{0}}(0,0) .
$$

As to the r.h.s. contribute only elements of type $(0, m)$ from $H^{\bullet}\left(\left(\text { Ass }_{\infty}\right)^{\circlearrowright}\right)$ and elements of type $(n, 0)$ from $H^{\bullet}\left(\left(\text { Ass }_{\infty}\right)^{\circlearrowright}\right)^{\dagger}$, the required result follows from Theorem 6.1.1.

6.4. Proof of Corollary D (see $\S 1$ ). By Theorems C and 6.1.1, $H^{\bullet}\left(\mathfrak{G}_{g}\right)$, is isomorphic to the vector space spanned by all possible directed $(0,0)$-graphs which can be obtained by gluing input legs of a disjoint union of $(0, n)$-graphs of the types,

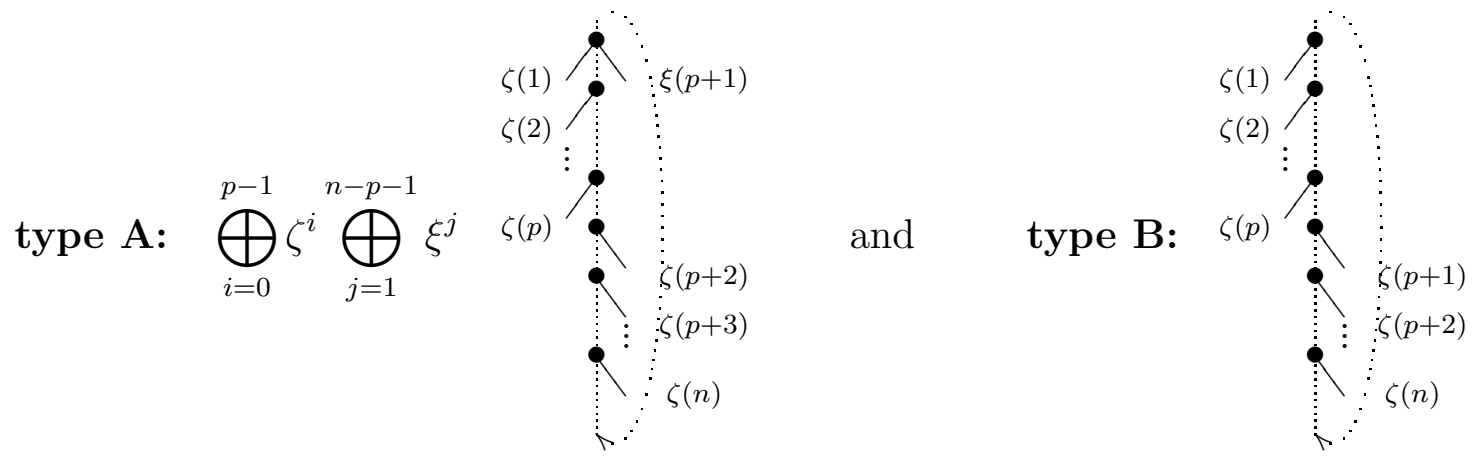

with output legs of a disjoint union of similar $(m, 0)$-graphs of the corresponding types $\bar{A}$ and $\bar{B}$.

Thus with every nonzero element $e$ of $H^{\bullet}\left(\mathfrak{G}_{g}\right)$ we can associate

\footnotetext{
${ }^{5}$ see $\S 3.11$ in 24 for the definition of the operadic wheelification functor.
} 
- a collection of $p$ graphs of type $A$ each having $a_{i}$ vertices (and hence $a_{i}+1$ input legs), $1 \leq i \leq p$

- a collection of $q$ graphs of type $B$ each having $b_{j}$ vertices (and hence $b_{j}$ input legs), $1 \leq j \leq q$

- a collection of $\bar{p}$ graphs of type $\bar{A}$ each having $\bar{a}_{i}$ vertices (and hence $\bar{a}_{\bar{i}}+1$ output legs), $1 \leq \bar{i} \leq \bar{p}$

- a collection of $\bar{q}$ graphs of type $\bar{B}$ each having $\bar{b}_{\bar{j}}$ vertices (and hence $\bar{b}_{\bar{i}}$ output legs), $1 \leq \bar{j} \leq \bar{q}$

such that total number, say $N$, of input legs of graphs of types $A$ and $B$ is equal to the total number of output legs of graphs of types $\bar{A}$ and $\bar{B}$, i.e.

$$
N=\sum_{i=1}^{p} a_{i}+\sum_{j=1}^{q} b_{j}+p=\sum_{\bar{i}=1}^{\bar{p}} \bar{a}_{\bar{i}}+\sum_{\bar{j}=1}^{\bar{q}} \bar{b}_{\bar{j}}+\bar{p} .
$$

The total number of internal edges of a graph $G$ obtained from the above data by gluing $N$ output legs with $N$ input legs is equal to $3 N-p-\bar{p}$ while the total number of vertices is $2 N-p-\bar{p}$. By the Euler formula, the genus of $G$ is

$$
g=1+(3 N-p-\bar{p})-(2 N-p-\bar{p})=N+1
$$

Thus our non-zero element $e$ belongs to $H^{2 g-2-p-\bar{p}}\left(\mathfrak{G}_{g}\right)$ and we immediately conclude that $H^{n}\left(\mathfrak{G}_{g}\right)=0$ for all $n>2 g-2$.

It is clear from (48) that, for fixed $N=g-1$, the maximal possible values of parameters $p$ and $\bar{p}$ are equal to $[N / 2]$. Hence $H^{n}\left(\mathfrak{G}_{g}\right)$ is indeed non-zero only for $n$ in the range $g-\frac{1}{2}\left(1-(-1)^{g}\right) \leq$ $n \leq 2(g-1)$.

6.4.1. Example. $H^{2}\left(\mathfrak{G}_{3}\right)$ is two dimensional and is spanned by the following graphs,
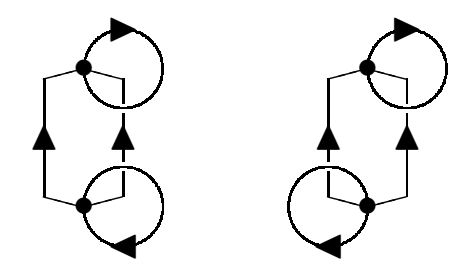

6.5. Proof of Theorem A (see $§ 1$ ). We have to show that the natural morphism of dg wheeled properads,

$$
p:\left(\operatorname{Ass}_{\infty}^{\circlearrowright}, \partial\right) \longrightarrow\left(\text { Ass }^{\circlearrowright}, 0\right)
$$


given on generators by

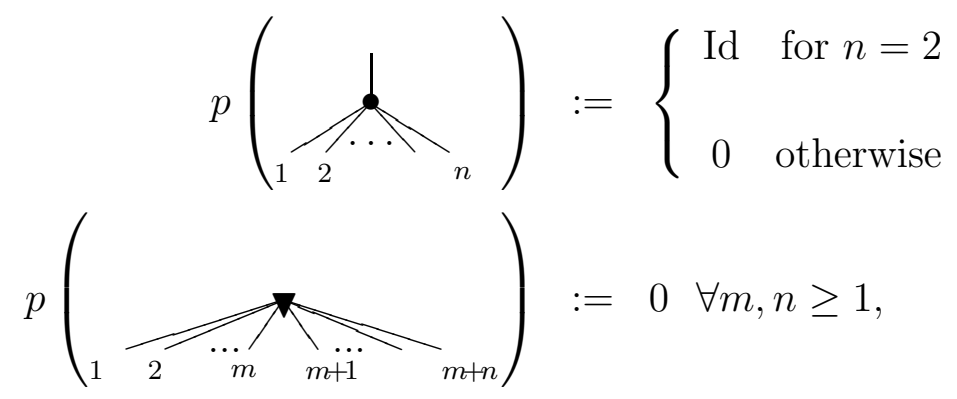

is a quasi-isomorphism.

Consider an increasing filtration, $F_{0} \subset F_{1} \subset \ldots \subset F_{p} \subset \ldots$ of the complex (Ass $\left.{ }_{\infty}^{\circlearrowright}, \partial\right)$ by the number $p$ of $\bullet$-vertices, and let $\left\{E_{r}, \delta_{r}\right\}_{r \geq 0}$ be the associated spectral sequence. The differential $\delta_{0}$ is non-zero only on $\boldsymbol{\nabla}$-vertices, and is given by

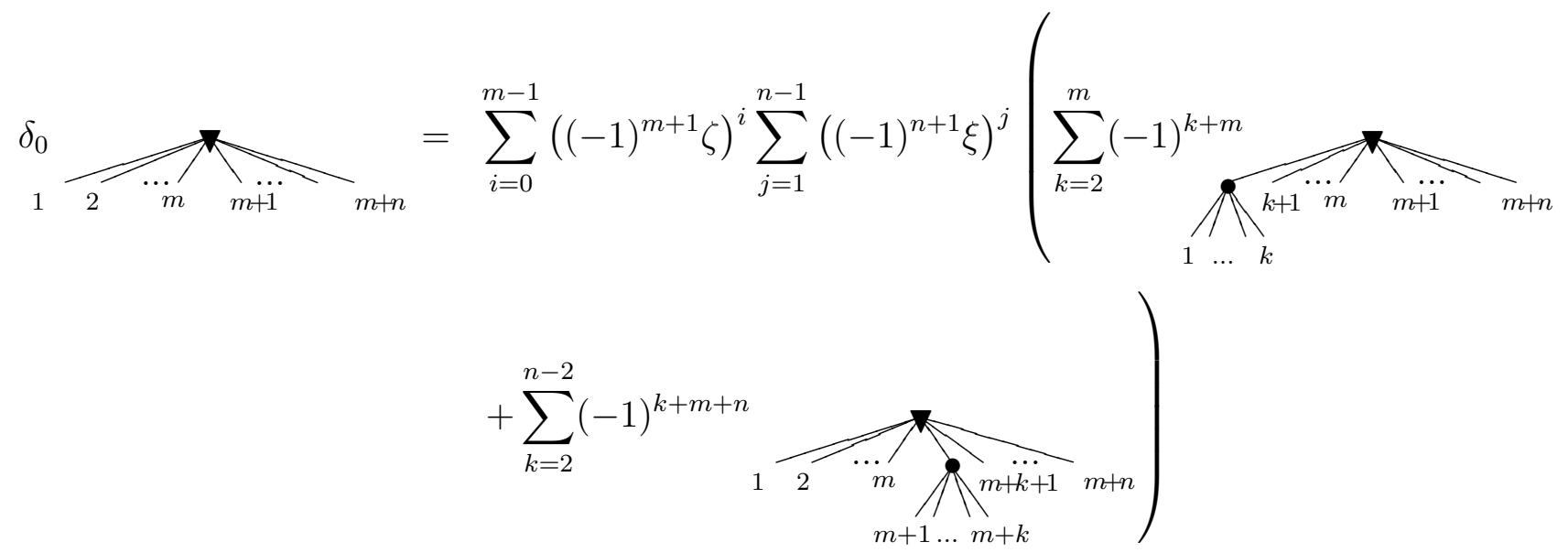

Let $\left(C C_{-*}(W), \partial_{W}\right)$ be the (negatively graded) cellular chain complex of the cyclohedron introduced and studied in [18]. A comparison of the differential $\delta_{0}$ with the boundary operator $\partial_{W}$ (given explicitly in Proposition 2.14 of [18]) immediately implies that, modulo action of finite groups, the complex $\left(E_{0}, \delta_{0}\right)$ is isomorphic to the tensor product of a trivial complex (i.e. one with vanishing differential) with the complex $\mathbb{R}\left[\Sigma_{*+\bullet+2}\right] \otimes \downarrow C C_{-*}(W) \otimes \downarrow C C_{-\bullet}(W)$. As cyclohedron consists of contractible polytopes, its homology is concentrated in degree 0 and is equal to $\mathbb{R}$. Thus $\left(E_{1}, \delta_{1}\right)$ is the free wheeled properad generated by •-corollas and extra binary corollas in degree -2 ,

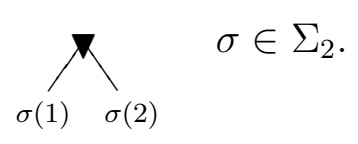

standing for the natural basis of $\mathbb{R}\left[\Sigma_{2}\right] \otimes H^{0}\left(\downarrow C C_{-*}(W) \otimes \downarrow C C_{-*}(W)\right)=\downarrow^{2} \mathbb{R}\left[\Sigma_{2}\right]$.

The induced differential $\delta_{1}$ is given on •-corollas by the usual $A_{\infty}$ formula, while on the remaining binary $\boldsymbol{\nabla}$-corollas as 


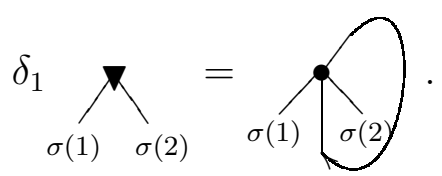

By Theorem 6.1.1 and Proposition 6.1.2, $E_{2}$ is precisely Ass ${ }^{\circlearrowright}$. Hence the induced differential $\delta_{2}$ vanishes so that converging to $H\left(\mathrm{Ass}_{\infty}^{\circlearrowright}, \partial\right)$ spectral sequence $\left\{E_{r}, d_{r}\right\}$ degenerates at this second term. This completes the proof.

6.6. Wheeled Massey operations. Let $(V, \cdot d)$ be a differential graded associative algebra over a field $\mathbf{k}$, and let $(W, d)$ be a complex together with morphisms of complexes, $i: W \rightarrow V$ and $p: V \rightarrow W$ such that

$$
p \circ i=\mathrm{Id}+Q d+d Q
$$

for some degree -1 linear operator $Q: V \rightarrow V$. Homotopy theory general nonsense says that $W$ must have an induced Ass $_{\infty}$-structure, $\left\{\mu_{n}: W^{\otimes n} \rightarrow \uparrow^{n-2} W\right\}_{n \geq 1}$, and the following explicit formulae for this structure have been found in [21]:

$$
\begin{aligned}
& \mu_{1}=d, \\
& \mu_{n}=p \circ \lambda_{n} \circ i^{\otimes n} \quad \text { for } n \geq 2,
\end{aligned}
$$

where $\lambda_{n}: V^{\otimes n} \rightarrow \uparrow^{n-2} V$ are defined inductively as follows,

$$
\lambda_{n}\left(v_{1}, \ldots, v_{n}\right):=\sum_{\substack{k+l=n+1 \\ k, l \geq 1}}(-1)^{k+(l-1)\left(\left|v_{1}\right|+\ldots+\left|v_{n}\right|\right)} Q \lambda_{k}\left(v_{1}, \ldots, v_{k}\right) \cdot Q \lambda_{n-k}\left(v_{k+1}, \ldots, v_{n}\right),
$$

starting with formal equality $\lambda_{1}:=-Q^{-1}$.

If $V$ is finite dimensional, then the datum $(V, \cdot d)$ is canonically an Ass ${ }^{\circlearrowright}$-algebra so that again we may expect that $W$ (which may not be finite-dimensional!) has a naturally induced Ass $_{\infty}^{\circlearrowright}$-structure,

$$
\left\{\mu_{n}: W^{\otimes n} \rightarrow \uparrow^{n-2} W\right\}_{n \geq 1}, \quad\left\{\mu_{p, q}: W^{\otimes p+q} \rightarrow \uparrow^{p+q} \mathbf{k}\right\}_{p, q \geq 1},
$$

which satisfy quadratic equations mimicking formulae (1) and (2) respectively. Straightforward but very tedious calculations show that this is indeed so with $\mu_{n}$ given by the formulae above and the new set of operations $\mu_{p, q}$ given as follows: if $\operatorname{Tr}_{i}\left(Q \circ \lambda_{n}\right): V^{\otimes n-1} \rightarrow \uparrow^{n-1} \mathbf{k}$ stands for the trace of the linear map $Q \circ \lambda_{n}: V^{\otimes n} \rightarrow \uparrow^{n-1} V$ with respect to the $i$-th input, $2 \leq i \leq n-1$, $n \geq 3$, then one has

$$
\begin{aligned}
& \mu_{i-1, n-i}\left(w_{1}, \ldots, w_{i-1}, w_{i+1}, \ldots w_{n}\right)= \\
= & \frac{1}{2} \sum_{l=0}^{i-2} \sum_{j=1}^{n-i-1}(-1)^{l i+1+(n-i+1) j} \operatorname{Tr}_{i}\left(Q \circ \lambda_{n}\right)\left(i\left(w_{\zeta^{l}(1)}\right), \ldots, i\left(w_{\zeta^{l}(i-1)}\right), i\left(w_{\xi^{j}(i+1)}\right), \ldots, i\left(w_{\xi^{j}(n)}\right)\right)
\end{aligned}
$$


where

$$
\zeta:=(12 \ldots i-1) \in \Sigma_{i-1}, \quad \xi:=((i+1)(i+2) \ldots n) \in \Sigma_{n-i}
$$

and $w_{\bullet}$ are arbitrary elements of $W$.

In particular, the cohomology of any finite dimensional dg associative algebra is naturally an $\mathrm{Ass}_{\infty}^{\circlearrowright}$-algebra and hence admits, in general, lots of new Massey type cyclically (skew)symmetric operations corresponding to compositions $\mu_{p, q}$.

6.7. Cyclic characteristic class of an $\mathrm{Ass}_{\infty}$-algebra. Any finite dimensional representation of the operad Ass extends naturally to a representation of the wheeled properad Ass ${ }^{\circlearrowright}$. It is not true, however, that any finite dimensional representation of the $\mathrm{dg}$ operad Ass $_{\infty}$ can be extended to a representation of the $\mathrm{dg}$ wheeled properad $\mathrm{Ass}_{\infty}^{\circlearrowright}$ - there exist a cohomological obstruction, and the main purpose of this subsection is to describe it, that is, to associate with an arbitrary finite dimensional Ass $_{\infty}$-algebra a cohomology class whose vanishing provides a necessary and sufficient condition for existence of its wheeled extension.

It is well known that with any graded vector space $V$ one can associate a graded Lie algebra

$$
C^{\bullet}(V, V)=\bigoplus_{n} C^{n}(V, V), \quad C^{n}(V, V):=\bigoplus_{j \geq 1} H_{o m} m_{n+1}\left((\uparrow V)^{\otimes j}, V\right)
$$

equipped with the Gerstenhaber Lie bracket, $[,]_{G}$. Here $H_{o m}\left(V^{\otimes j}, V\right)$ stands for the space of homogeneous maps $V^{\otimes j} \rightarrow V$ of degree $i$.

Maurer-Cartan elements in this Lie algebra, that is, elements $\Gamma \in C^{1}(V, V)$ such that $[\Gamma, \Gamma]_{G}=$ 0 , are in 1-1 correspondence with Ass $_{\infty}$-structures in $V$. Such elements make also $\left.(C \bullet V, V),[,]_{G}\right)$ into a differential graded Lie algebra, $\left(C^{\bullet}(V, V),[,]_{G}, D_{\Gamma}\right)$, with $D_{\Gamma}:=[\Gamma,]_{G}$.

Define next a graded vector space,

$$
C y c^{\bullet}(V, \mathbf{k}):=\bigoplus_{n} C y c^{n}(V, \mathbf{k}), \quad C y c^{n}(V, \mathbf{k}):=\bigoplus_{p, q \geq 1} H_{o m}\left(\left((\downarrow V)^{\otimes p}\right)_{C_{p}} \otimes\left((\downarrow V)^{\otimes q}\right)_{C_{q}}, \mathbf{k}\right) .
$$

It is naturally a module over the graded Lie algebra $\left(C^{\bullet}(V, V),[,]_{G}\right)$ so that the direct sum,

$$
\mathfrak{g}:=C^{\bullet}(V, V) \bigoplus C y c^{\bullet}(V, \mathbf{k}),
$$

has structure of a graded Lie algebra with the Lie bracket defined by

$$
[a, b]:=[a, b]_{G}, \quad[a, x]:=a \circ x, \quad[x, y]=0
$$

for arbitrary $a, b \in C^{\bullet}(V, V), x, y \in C y c^{\bullet}(V, \mathbf{k})$. Here o stands for the action of $C^{\bullet}(V, V)$ on $C y c^{\bullet}(V, \mathbf{k})$.

Any Maurer-Cartan element $\Gamma \in C^{1}(V, V)$ defines a Maurer-Cartan element, $\Gamma \oplus 0$, in $\mathfrak{g}$ and hence makes the latter and, in particular, the subspace $C y c^{\bullet}(V)$ into a complex with the differential

$$
\delta_{\Gamma}:=[\Gamma \oplus 0, \quad]
$$


6.7.1. Remark. In fact, general Maurer-Cartan elements in $\mathfrak{g}$ describe strongly homotopy Ass ${ }^{\omega}$ algebras, where Ass ${ }^{\omega}$-structures consist of an associative multiplication $\mu: V \otimes V \rightarrow V$ and an operation $\omega: V \otimes V \rightarrow \mathbf{k}$ such that, besides the associativity of $\mu, \omega(\mu(a, b), c)$ is symmetric in $a, b$ and $\omega(a, \mu(b, c))$ is symmetric in $b, c$.

If $V$ is a finite dimensional vector space, then we can associate with the Maurer-Cartan element, $\Gamma:=\left\{\mu_{n}: V^{\otimes n} \rightarrow \uparrow^{n-2} V\right\}_{n \geq 1} \in C^{1}(V, V)$, an element

$$
\Gamma^{\circlearrowright}:=\left\{\Gamma_{p, q}^{\circlearrowright} \in \operatorname{Hom}_{1}\left(\left((\downarrow V)^{\otimes p}\right)_{C_{p}} \otimes\left((\downarrow V)^{\otimes q}\right)_{C_{q}}, \mathbf{k}\right)\right\}_{p, q \geq 1} \in C y c^{1}(V, \mathbf{k}),
$$

as follows,

$$
\begin{aligned}
& \Gamma_{p, q}\left(v_{1}, \ldots, v_{p}, v_{p+1}, \ldots v_{p+q}\right):= \\
& \quad=\sum_{i=0}^{p-1} \sum_{j=1}^{q-1}(-1)^{i(p+1)+j(q+1)} \operatorname{Tr}_{p+1}\left(\mu_{p+q+1}\right)\left(v_{\zeta^{l}(1)}, \ldots, v_{\zeta^{l}(p)}, v_{\xi^{j}(p+1)}, \ldots, v_{\xi^{j}(p+q)}\right)
\end{aligned}
$$

where $\zeta:=(12 \ldots p) \in \Sigma_{p}, \xi:=((p+1)(p+2) \ldots(p+q)) \in \Sigma_{q}, \operatorname{Tr}_{p+1}\left(\mu_{p+q+1}\right): V^{\otimes p+q} \rightarrow \uparrow^{n-2} \mathbf{k}$ is the trace of the linear map $\mu_{p+q+1}: V^{p+q+1} \rightarrow \uparrow^{p+q-2} V$ with respect to the $(p+1)$-st input, and $v_{1}, \ldots, v_{p+q}$ are arbitrary elements of $V$.

6.7.2. Proposition-definition. For any finite-dimensional Ass $_{\infty}$-algebra $(V, \Gamma)$ we have

$$
\delta_{\Gamma} \Gamma^{\circlearrowright}=0 .
$$

The associated cohomology class $\left[\Gamma^{\circlearrowright}\right] \in H^{1}\left(C y c^{\bullet}(V, \mathbf{k})\right)$ is called the cyclic characteristic class of the $\operatorname{Ass}_{\infty}$-algebra $(V, \Gamma)$.

Sketch of a proof. It follows from definition (1) of the differential in the operad Ass $\infty$ that
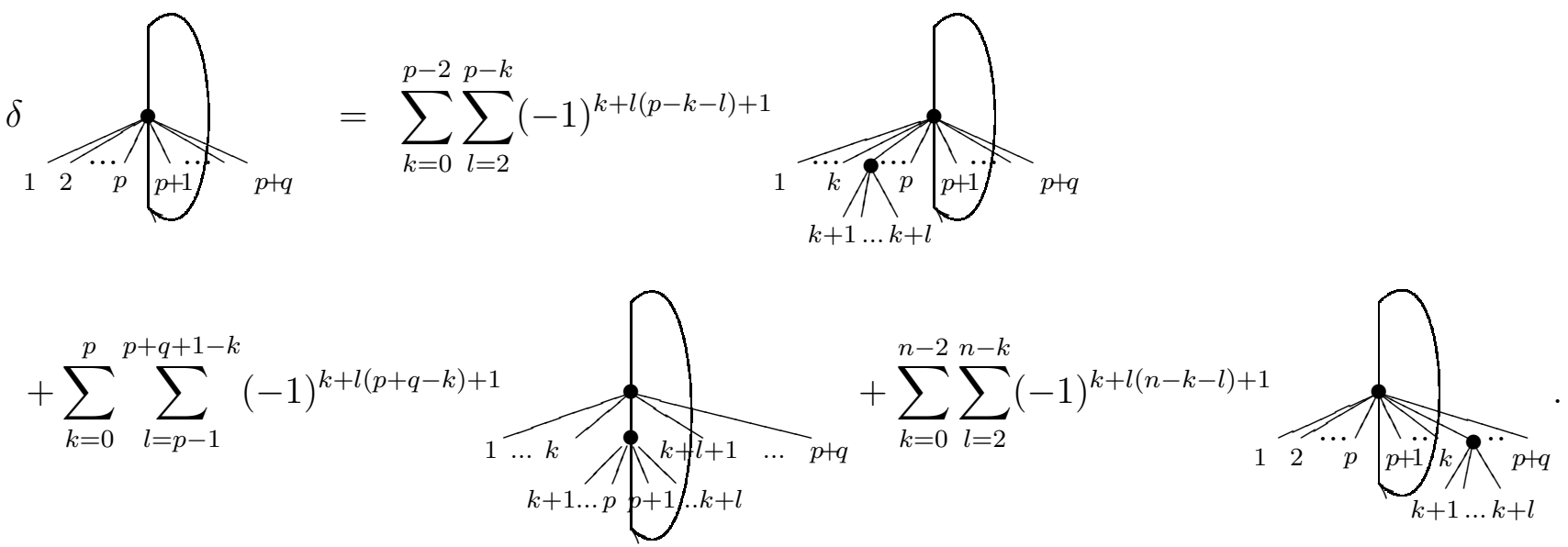

Straightforward calculations show that upon "(skew)cyclization" of both sides of the above equality as in (49) the middle sum (consisting of graphs with two cyclic vertices) vanishes. Then the remaining terms, when represented in a vector space $V$, assemble precisely into the required equation $\delta_{\Gamma} \Gamma^{\circlearrowright}=0$. 
6.7.3. Theorem. An Ass $_{\infty}$-structure, $\Gamma:=\left\{\mu_{n}: V^{\otimes n} \rightarrow \uparrow{ }^{n-2} V\right\}_{n \geq 1}$, in a finite-dimensional vector space $V$ can be extended to an $\mathrm{Ass}_{\infty}^{\circlearrowright}$-structure if and only if the cyclic characteristic class $\left[\Gamma^{\circlearrowright}\right]$ vanishes.

Proof. If the cyclic characteristic class vanishes, then $\Gamma^{\circlearrowright}=\delta_{\Gamma} \Theta$ for some $\Theta=\left\{\mu_{p, q}: V^{\otimes p+q} \rightarrow \uparrow\right.$ $\left.{ }^{p+q} V\right\}_{p, q \geq 1} \in C y c^{0}(V, \mathbf{k})$. Hence the datum $\left\{\mu_{\bullet}, \mu_{\bullet, \bullet}\right\}$ makes $V$ into an Ass ${ }_{\infty}^{\circlearrowright}$-algebra.

On the other hand, if $\left\{\mu_{\bullet}, \mu_{\bullet}, \bullet\right\}$ is an Ass $_{\infty}^{\circlearrowright}$-structure on $V$, then $\Theta:=\left\{\mu_{p, q}: V^{\otimes p+q} \rightarrow\right.$ $\left.\uparrow^{p+q} V\right\}_{p, q \geq 1} \in \operatorname{Cyc}^{0}(V, \mathbf{k})$ satisfies the equation $\delta_{\Gamma} \Theta=\Gamma^{\circlearrowright}$.

A unusual feature of the situation described in Theorem 6.7.2. is that there exists precisely one obstruction to extension of $\mathrm{Ass}_{\infty}$-algebras into $\mathrm{Ass}_{\infty}^{\circlearrowright}$-algebras while in obstruction theory one usually has to deal with an infinite series of obstructions. The explanation is, however, simple the wheeled operad $\mathrm{Ass}_{\infty}^{\circlearrowright}$ has only elements of genus 0 or 1.

6.7.4. Example. There is an interesting class of Ass $_{\infty^{-}}$-algebras which always extend into Ass $_{\infty^{-}}$algebras, consisting of structures $\Gamma:=\left\{\mu_{n}: V^{\otimes n} \rightarrow \uparrow^{n-2} V\right\}_{n \geq 1}$ such that the antisymmetrization of the trace $\operatorname{Tr}_{p+1}\left(\mu_{p+q+1}\right): V^{\otimes p+q} \rightarrow \uparrow^{n-2} \mathbf{k}$ in the first $p$ and the last $q$ variables vanishes for each $p, q \geq 1, n=p+q$. We suggest to call these algebras traceless Ass $_{\infty}$-algebras.

\section{WheEled Com}

In this section we prove Theorem B of the introduction and show thereby that the operad Com for commutative associative algebras is wheeled Koszul. We also demonstrate directly that Com is not stably Koszul in the sense of Definition 5.2 .10 by calculating, in Theorem 7.1 .1 , the homology of the wheeled completion $\left(\mathrm{Com}_{\infty}\right)^{\circlearrowright}$.

7.1. Resolution of wheeled Com. The operad Com is the quotient

$$
\text { Com }=\Gamma_{o p}\langle E\rangle /(R),
$$

of the free operad $\Gamma_{o p}\langle E\rangle$ on the $\Sigma$-module $E$ with

$$
\begin{array}{ll}
E(2):=\mathbb{1}_{2} & \text { spanned by } \bigcap_{1}=\overbrace{2} \\
E(n):=0, & n \neq 1
\end{array}
$$

modulo the ideal generated by the relations

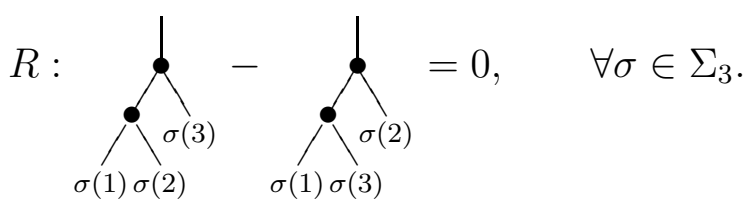

Recall that the minimal model Com $_{\infty}$ of the operad Com is generated by standard associative $(1, n)$-corollas in degree $2-n, n \geq 2$, modulo the shuffle relations as in Theorem $\mathrm{B}(\mathrm{i})$, with the differential given by (41). 
7.1.1. Theorem. The dimension of the homology of $\left(\operatorname{Com}_{\infty}\right)^{\circlearrowright}(0, n)$ in degree $-k \leq 0$ equals $\left(\begin{array}{c}n-1 \\ k\end{array}\right)$.

In the above theorem which we prove in Subsection 7.4 we used the convention that $\left(\begin{array}{l}n \\ k\end{array}\right)=0$ for $k>n$. Theorem 7.1.1 shows that, in order to obtain a minimal model of Com ${ }^{\circlearrowright}$, one must add new generators to $\left(\mathrm{Com}_{\infty}\right)^{\circlearrowright}$ that kill homology classes in $H^{<0}\left(\left(\mathrm{Com}_{\infty}\right)^{\circlearrowright}, \partial\right)$, then add generators that kill relations among these new generators, etc. The result is described in Theorem B which we prove later in this section. We also prove:

\subsubsection{Theorem. The operad Com is wheeled Koszul.}

Theorem 7.1 .2 together with the uniqueness, up to isomorphism, of minimal models imply that the minimal model of $\mathrm{Com}^{\circlearrowright}$ described in Theorem B coincides with the wheeled cobar of the wheeled quadratic dual (Com $\left.{ }^{\circlearrowright}\right) !$ constructed in Example 5.2.4.

Theorem B and Theorem 7.1.2 are proved in Subsection 17.5, Their proofs are based on an analysis of $(\Omega(B(\mathrm{Com})))^{\circlearrowright}$ (the wheeled completion of the double bar complex for the ordinary Com $)$ and $\Omega^{\circlearrowright}\left(B^{\circlearrowright}\left(\mathrm{Com}^{\circlearrowright}\right)\right)$ (the double bar complex of $\mathrm{Com}^{\circlearrowright}$ in the category of wheeled operads).

7.2. Double bar complex of Com. Let us show how the description of Com $_{\infty}$ presented above can be read off from the ordinary double bar complex $\Omega(B($ Com $))$. Recall that $\Omega(B($ Com $))(n)$ is generated by trees with one output leg and $n$ input legs, with vertices the standard commutative corollas with one outgoing edge and $\geq 2$ symmetric incoming edges. The set of edges $\operatorname{Edg}(G)$ of each tree $G$ is two-colored (in pictures, black edges are ordinary ones, and white edges are doubled ones).

In the construction below, we use the same conventions as in the proof of Theorem 4.2.5, in particular, the bigrading (50) is the one used in the proof of Theorem 4.2.5. The space $\Omega(B($ Com $))(n)$ is bigraded, with the bidegree given by

$$
\operatorname{bideg}(G)=\left(\operatorname{deg}_{1}(G), \operatorname{deg}_{2}(G)\right):=\left(-e(G), e^{0}(G)\right)
$$

where $e(G)$ is the number of internal edges of the underlying graph $G$ and $e^{\circ}(G)$ the number of white edges of $G$. There are two differentials, $(1,0)$-and $(0,1)$-ones, denoted by $\partial_{1}$ and $\partial_{2}$, respectively. The first one contracts black edges, and the second one changes the color of edges from black to white. For example, 


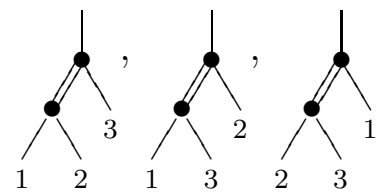

$\Omega(B(\mathrm{Com}))(3)$ :

$\partial_{2} \uparrow$

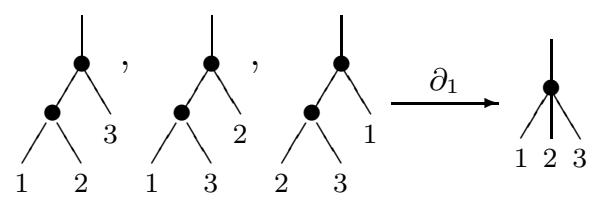

Since Com is Koszul, its $\partial_{1}$-cohomology of $\Omega(B($ Com $))$ is concentrated in bidegrees $(2-n, *)$ and is isomorphic to $\mathrm{Com}_{\infty}$ (the differential of $\mathrm{Com}_{\infty}$ is induced by $\partial_{2}$ ). The description of $\mathrm{Com}_{\infty}$ given above uses associative corollas satisfying shuffle relations. They are represented in $\Omega(B($ Com $))$ in the following way. Consider the sum of all planar binary trees with only black edges with 1 output leg and $n$ input legs marked by $\sigma(1), \ldots, \sigma(n)$ from the left to the right. There is, modulo an overall sign, a unique choice of signs of the graphs such that this sum is a $\partial_{1}$-closed element in $\Omega(B(\mathrm{Com})(n)$. It represents the associative corolla with $n$ inputs marked by $\sigma(1), \ldots, \sigma(n)$ from the left to the right. One can check that these corollas satisfy the shuffle relations, and that $\partial$ is induced by $\partial_{2}$.

Here are few examples (we omit the signs; associative corollas are on the left, commutative corollas are on the right):

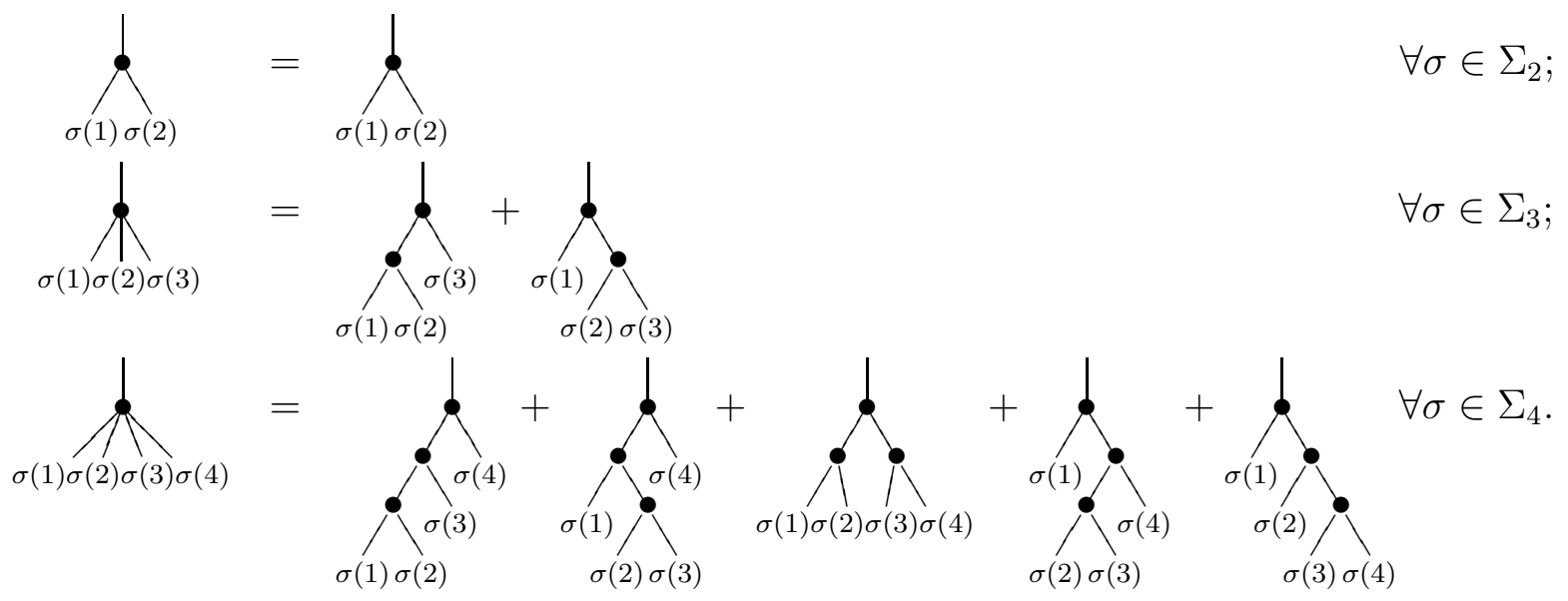

7.3. Wheeled double bar complexes. We consider three wheeled dg-operads whose operadic parts coincide with $\Omega(B(\mathrm{Com}))$. The first one is its wheeled completion $\left(\Omega(B(\mathrm{Com}))^{\circlearrowright}\right.$. The space $\left(\Omega(B(\mathrm{Com}))^{\circlearrowright}(0, n)\right.$ is spanned by all graphs with one oriented wheel and $n$ input legs. There are black and white edges, as before, but we require that there is at least one white edge in the wheel. Bigrading and differentials are extended from $\Omega(B($ Com $))$. 


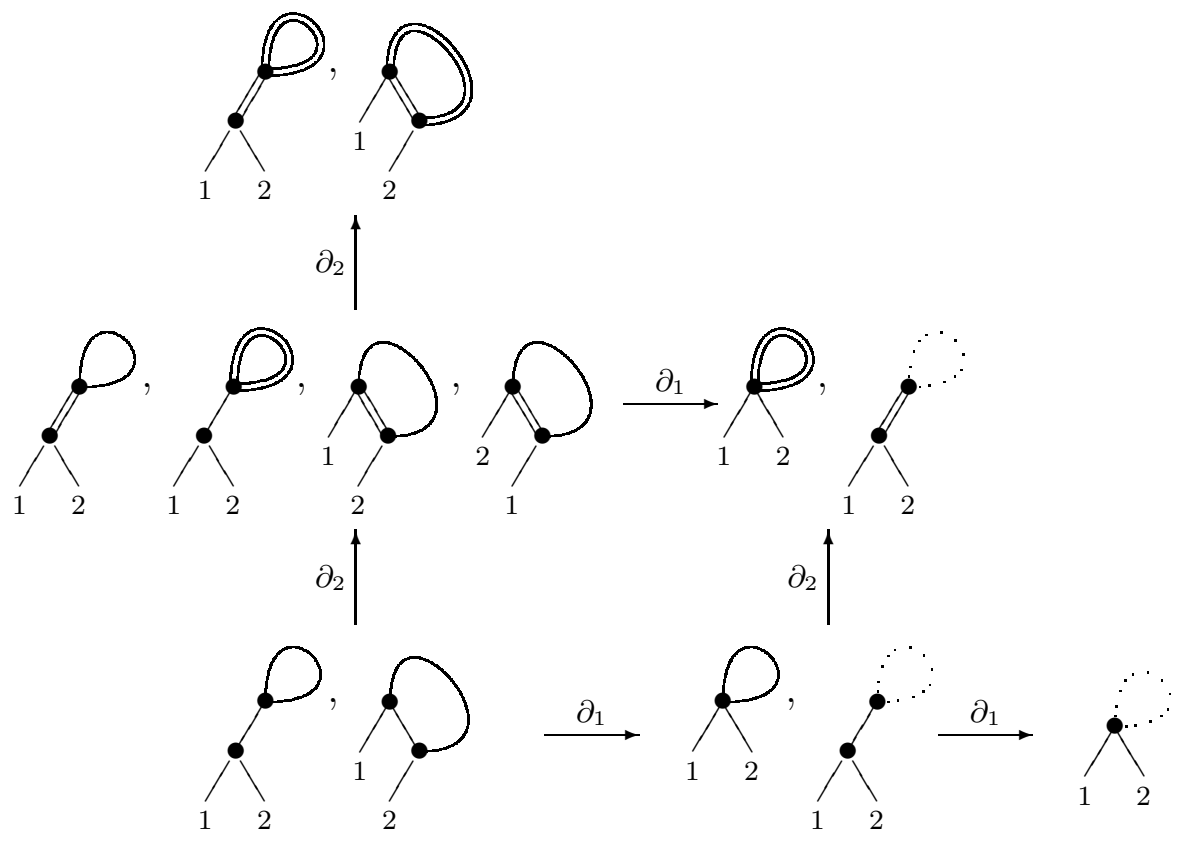

Figure 7. The bicomplex $\Omega^{\circlearrowright}\left(B^{\circlearrowright}\left(\mathrm{Com}^{\circlearrowright}\right)\right)(0,2)$.

The second one is the extended wheeled completion denoted by $\left(\Omega(B(\text { Com }))_{x}^{\circlearrowright}\right.$ defined as the extension of $\left(\Omega(B(\mathrm{Com}))^{\circlearrowright}\right.$ by graphs that have all edges in the wheel black. Notice that $\left(\Omega(B(\mathrm{Com}))_{x}^{\circlearrowright}\right.$ contains also graphs with black loops (one-edge wheels). Applying $\partial_{1}$ to such graphs would require to contract such loops. But this is impossible in $\left(\Omega(B(\text { Com }))_{x}^{\circlearrowright}\right.$ and we extend the differential by postulating that contracting such a loop gives zero.

We can consider one more wheeled dg-operad where this contraction is defined. We add graphs with one-edge wheel considered as a "virtual" one (indicated by dots in pictures). Obviously, what we get (with naturally extended gradings and differentials) is the wheeled double bar complex $\Omega^{\circlearrowright}\left(B^{\circlearrowright}\left(\mathrm{Com}^{\circlearrowright}\right)\right)$.

The example of $\Omega^{\circlearrowright}\left(B^{\circlearrowright}\left(\mathrm{Com}^{\circlearrowright}\right)\right)(0,2)$ is given in Figure 7. The bicomplex $\left(\Omega(B(\mathrm{Com}))_{x}^{\circlearrowright}(0,2)\right.$ is a quotient of the above one consisting of graphs with no virtual loops; $\left(\Omega(B(\mathrm{Com}))^{\circlearrowright}(0,2)\right.$ is a sub-bicomplex without virtual loops and fully black wheels.

\subsubsection{Proposition. The complex $\left(\Omega(B(\mathrm{Com}))_{x}^{\circlearrowright}(0, n)\right.$ is, for each $n \geq 1$, acyclic.}

Proof. We use the same reasoning as in the proof of Theorem 4.2.5. As before, $\left(\Omega(B(\text { Com }))_{x}^{\circlearrowright}(0, n)\right.$ with differential $\partial_{2}$ splits into the direct sum over isomorphism classes of graphs of complexes of exterior algebras (25) on edges of each type of graph. It is acyclic if there is at least one edge in the underlying graph. But here each graph has at least one edge. Therefore, $\left(\Omega(B(\text { Com }))_{x}^{\circlearrowright}(0, n)\right.$ is acyclic. 
7.4. Proof of Theorem 7.1.1. Notice that the homology of $\left(\mathrm{Com}_{\infty}\right)^{\circlearrowright}$ coincides with the homology of $(\Omega(B(\mathrm{Com})))^{0}$. This follows from the fact that the free wheeled operad functor is a polynomial functor on the category of $\Sigma$-modules and that the wheeled completion of a free operad is a free wheeled operad, so the wheeled completions of free operads generated by quasiisomorphic $\mathrm{dg}$ - $\Sigma$-modules are quasi-isomorphic.

Since $(\Omega(B(\text { Com })))^{\circlearrowright}$ is a subcomplex of the acyclic complex $(\Omega(B(\text { Com })))_{x}^{\circlearrowright}$, it is enough to study their quotient,

$$
\mathcal{F}_{x / c}:=\frac{(\Omega(B(\mathrm{Com})))_{x}^{\circlearrowright}}{(\Omega(B(\mathrm{Com})))^{\circlearrowright}}
$$

Note that $\mathcal{F}_{x / c}$ is generated by graphs with fully black wheels. The first differential, $\partial_{1}$, is the same as before and contracts black edges. The second differential, $\partial_{2}$, changes the color of edges outside the wheel from black to white.

The $\partial_{2}$-cohomology of $\mathcal{F}_{x / c}$ splits into the direct sum of complexes of exterior algebras on edges, as in the proofs of Theorem 4.2.5 and Proposition 7.3.1. But here we consider only the edges outside the wheel, therefore generators of the $\partial_{2}$-cohomology are graphs with no edges outside the wheel.

It follows from a short exact sequence argument and the identification above that the cohomology of $\left(\operatorname{Com}_{\infty}\right)^{\mathcal{O}}(0, n)$ shifted by 1 equals to the cohomology of the graph complex $\mathcal{W}_{n}$ spanned by wheels with $n$ numbered legs, with no edge outside the wheel, and with the differential $\partial_{1}$ contracting edges. For example, $\mathcal{W}_{2}$ :

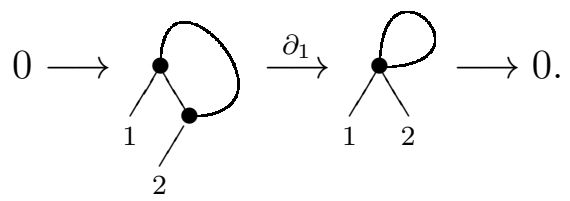

The grading, given by the number of edges, comes from the double bar complex. Observe that $\mathcal{W}_{n}$ is concentrated in degrees $-1, \ldots,-n$. To describe the differential $\partial_{1}$, it is convenient to consider graphs $G$ in $\mathcal{W}_{n}$ twisted by the determinant space $\operatorname{Det}(E d g(G)):=\Lambda^{|E d g(G)|}(\langle E d g(G)\rangle)$. Then

$$
\partial_{1}: \operatorname{Det}(E d g(G)) \otimes G \mapsto \sum_{e \in E d g(G)} \frac{\partial}{\partial e} \operatorname{Det}(E d g(G)) \otimes \partial_{e}(G),
$$

where $\partial_{e}(G)$ is, as before, the graph $G / e$ obtained by contraction of the edge $e$. If $e$ is a loop (i. e., $G$ is one-edge graph in degree -1 ), then $\partial_{e}(G)=0$. In particular, $\partial_{1} \equiv 0$ in $\mathcal{W}_{1}$.

Theorem 7.1.1 now follows from the following proposition whose proof is an exercise on cyclic cohomology:

7.4.1. Proposition. The dimension of $H^{-k}\left(\mathcal{W}_{n}\right)$ equals $\left(\begin{array}{c}n-1 \\ k-1\end{array}\right),-k \leq-1$.

The relation to cyclic cohomology is the following. The complex defining cyclic cohomology of the polynomial algebra in variables $x_{1}, \ldots, x_{n}$ contains a subcomplex generated by cyclic expressions where each $x_{i}$ appears exactly once. This subcomplex is isomorphic to $\mathcal{W}_{n}$. 
7.5. Proofs of Theorem B and Theorem 1.1.2. We prove Theorem 7.1.2 first. Let us consider $B^{\circlearrowright}\left(\mathrm{Com}^{\circlearrowright}\right)(0, n)$. It is the complex of graphs with $n$ input legs and no output legs whose vertices are decorated by elements in $\mathrm{Com}^{\circlearrowright}$, graded by the number of edges. The differential $\partial$ contracts the edges, introducing appropriate signs. For example,

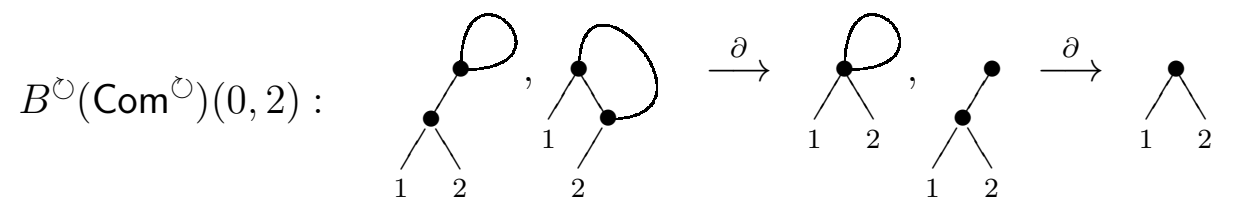

By definition of wheeled Koszulness, Theorem 7.1.2 is equivalent to the statement that the cohomology of $B^{\circlearrowright}\left(\operatorname{Com}^{\circlearrowright}\right)(0, n)$ is zero for $n=1$ and concentrated in degree $-n$ for $n \geq 2$, i.e., that it is generated by trivalent graphs of genus 1 .

For $n=1$ it follows from a direct inspection that $B^{\circlearrowright}\left(\mathrm{Com}^{\circlearrowright}\right)(0,1)$ is acyclic. If $n \geq 2$, we introduce a filtration on $B^{\circlearrowright}\left(\mathrm{Com}^{\circlearrowright}\right)(0, n)$ :

$$
0=F_{-1} \subset F_{0} \subset \cdots \subset F_{n}=B^{\circlearrowright}\left(\operatorname{Com}^{\circlearrowright}\right)(0, n),
$$

in which $F_{i}$ is the space of graphs with $\leq i$ edges in the wheel. For example, $F_{0}$ is the space of graphs with no wheel (i.e., there is one vertex with no output leg). Let us show that $H^{*}\left(F_{i} / F_{i-1}, \partial\right)=0$ for all $i<n$. Since $F_{n} / F_{n-1}$ is spanned by graphs in degree $-n$, this will, by an elementary spectral sequence argument, imply the statement.

Indeed, $F_{i} / F_{i-1}$ is spanned by graphs with exactly $i$ edges in the wheel. The differential induced by $\partial$ acts by contractions of edges outside the wheel. So, given a partition of the set $\{1, \ldots, n\}$ into $i$ subsets, $I_{1} \sqcup \cdots \sqcup I_{i}=\{1, \ldots, n\}$, we can consider a subcomplex in $F_{i} / F_{i-1}$ spanned by graphs such that, if we cut all edges in the wheel, we obtain $i$ graphs whose legs are marked by $I_{1}, \ldots, I_{i}$. Obviously, $F_{i} / F_{i-1}$ splits into the direct sum of such subcomplexes.

The graphs with legs marked by $I_{j}$ form a complex $\mathcal{C}\left(I_{j}\right)$. The subcomplex of $F_{i} / F_{i-1}$ corresponding to the partition $I_{1} \sqcup \cdots \sqcup I_{i}=\{1, \ldots, n\}$ is the direct sum of $(i-1)$ ! copies of $\bigotimes_{j=1}^{i} \mathcal{C}\left(I_{j}\right)$.

So, if we prove that at least one complex $\mathcal{C}\left(I_{j}\right)$ for $j=1, \ldots, i$ is acyclic, we immediately conclude that $F_{i} / F_{i-1}$ is acyclic. We will see that $\mathcal{C}\left(I_{j}\right)$ is acyclic if $\left|I_{j}\right| \geq 2$. This explains why $F_{i} / F_{i-1}$ is acyclic for $i<n$ (there must be at least one set $I_{j}$ with at least 2 elements; we assume $n \geq 2$ ) but it is not acyclic for $i=n$.

Consider $\mathcal{C}\left(I_{j}\right)$ assuming $\left|I_{j}\right| \geq 2$. It consists of trees with 1 output leg and $\left|I_{j}\right|+1$ input legs. We use the convention that the last input leg is the wheel input, attached to the same vertex (the special vertex) as the output leg (to handle $F_{0}$ in the same way, we would need to add a virtual loop at the vertex with no output leg). We introduce a two-term filtration $\mathcal{C}^{\prime}\left(I_{j}\right) \subset \mathcal{C}\left(I_{j}\right)$ such that $\mathcal{C}^{\prime}\left(I_{j}\right)$ consists of graphs whose the special vertex has biarity $(1, k), k>2$. It is easy to see that $\mathcal{C}^{\prime}\left(I_{j}\right)$ and $\mathcal{C}\left(I_{j}\right) / \mathcal{C}^{\prime}\left(I_{j}\right)$ are canonically isomorphic. Indeed, slightly abusing notations, 
we can say that $\mathcal{C}\left(I_{j}\right) / \mathcal{C}^{\prime}\left(I_{j}\right)$ is generated by graphs with the special vertex of biarity $(1,2)$. The isomorphism is then given by the contraction of the unique edge attached to the special vertex of a graph in $\mathcal{C}\left(I_{j}\right) / \mathcal{C}^{\prime}\left(I_{j}\right)$. The first level of the associated spectral sequence has two identical rows and the differential is an isomorphism of these rows. So, the cohomology vanishes at the next level of this spectral sequence. Therefore $F_{i} / F_{i-1}$ is acyclic for $i<n$ and Theorem 7.1 .2 is proved.

Now we can proceed with the proof of Theorem B. Wheeled Koszulness implies that the minimal resolution of $\mathrm{Com}^{\circlearrowright}$ is generated by the standard $(1, n)$-generators of $\mathrm{Com}_{\infty}$ and by some new $(0, n)$-generators which form a basis of $H^{-n}\left(B^{\circlearrowright}\left(\mathrm{Com}^{\circlearrowright}\right)(0, n)\right), n \geq 2$.

Our goal is to identify a basis of $H^{-n}\left(B^{\circlearrowright}\left(\operatorname{Com}^{\circlearrowright}\right)(0, n)\right), n \geq 2$, with the generators introduced in Theorem B(ii), and to show that the differential of the wheeled cobar construction on (Com ${ }^{\circlearrowright}$ )! coincides with (5). To do so, we study the wheeled double bar complex of $\mathrm{Com}^{\circlearrowright}$ in the same way as we analyzed the ordinary double bar complex of Com in Subsection 7.2 .

We define the bigrading of $\Omega^{\circlearrowright}\left(B^{\circlearrowright}\left(\operatorname{Com}^{\circlearrowright}\right)\right)(0, n)$ by $\operatorname{deg}_{1}(G)=-e(G)$ and $\operatorname{deg}_{2}(G)=e^{\circ}(G)$, where $e(G)$ denotes the number of all edges of the underlying graph $G$ and $e^{\circ}(G)$ the number of white edges of $G$ (it is the same bigrading as in the proof of Theorem 4.2.5). The nontrivial part of this bicomplex lies in the triangle bounded by $\operatorname{deg}_{1}=-n, \operatorname{deg}_{1}=-\operatorname{deg}_{2}$, and $\operatorname{deg}_{2}=0$ ( see example for $n=2$ in Subsection [7.3).

Consider the $\operatorname{deg}_{2}=0$ part of $\Omega^{\circlearrowright}\left(B^{\circlearrowright}\left(\mathrm{Com}^{\circlearrowright}\right)\right)(0, n)$ spanned by graphs with no white edges. Denote this subcomplex by $\mathcal{D}_{n}$. It follows from our constructions that $\left(\mathcal{D}_{n}, \partial_{1}\right)$ is isomorphic to $\left(B^{\circlearrowright}\left(\mathrm{Com}^{\circlearrowright}\right)(0, n), \partial\right)$.

To identify the generators of $H^{-n}\left(\mathcal{D}_{n}\right)$, we return to the argument with filtration $F_{*}$ from the proof of Theorem 7.1.2 (slightly abusing notations, we consider it on $\left(\mathcal{D}_{n}, \partial_{1}\right)$ ). Note that $\partial_{1}$ restricted to $F_{n} / F_{n-1}$ is equal to zero. So, there is a basis of $H^{-n}\left(\mathcal{D}_{n}\right), n \geq 2$, consisting of $(n-1)$ ! elements (we need to fix the cyclic order of legs markings), which are represented by wheels with no edges outside it, plus some correction terms arising from the spectral sequence associated to $F_{*}$. As in the case of the ordinary double bar complex, it is possible to give an explicit description of these correction terms.

Let us fix a cyclic order of the set $\{1, \ldots, n\}$. Consider the sum of all planar graphs of genus 1 with trivalent vertices such that: (i) all edges are black, (ii) the wheel contains at least two edges, (iii) the wheel is oriented in the clockwise direction and (iv) when we go around the graph in the counterclockwise direction, we meet the input legs in the prescribed cyclic order. There is, modulo an overall sign, a unique choice of signs of these graphs such that their sum is a $\partial_{1}$-closed element. 
This $\partial_{1}$-closed element is exactly what we denoted in Theorem B(ii) by

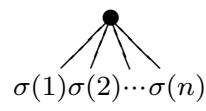

where $\sigma \in \Sigma_{n}$ determines the cyclic order (we identify elements that differ by a cyclic permutation). The first few examples are (we omit the signs):

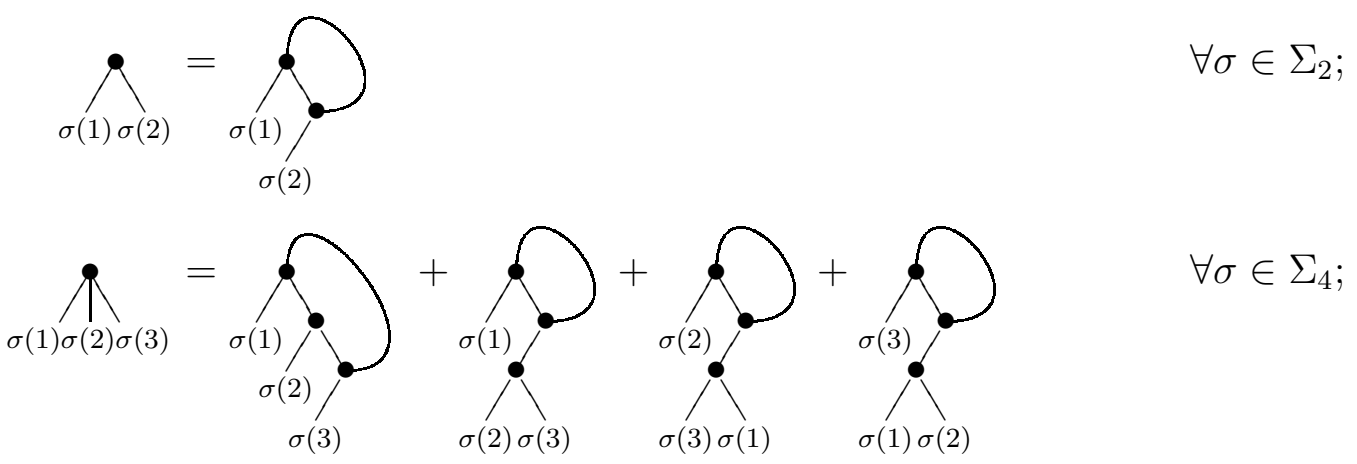

Up to signs, the formula for the differential of these new generators is almost obvious . The only subtlety is that, if we want $\partial_{2}$ to induce $\partial$ as in equation (5), we must consider also binary graphs of genus 1 satisfying conditions (i), (iii), and (iv), but with only one edge in the wheel. However, it is easy to check that we need to add each such a graph twice and with opposite signs. So, they do not affect the result.

Theorem B is therefore proved modulo signs. We leave the sign issue to the reader.

Acknowledgment. The first two authors would like to express their thanks to I.H.E.S. for a very stimulating and pleasant atmosphere, and acknowledge the support of the European Commission through its 6th Framework Programme "Structuring the European Research Area" and the contract Nr. RITA-CT-2004-505493 for the provision of Transnational Access implemented as Specific Support.

\section{REFERENCES}

[1] S. Barannikov. Generalized periods and mirror symmetry in dimensions $n \geq 3$. Preprint math.AG/9903124.

[2] S. Barannikov. Modular operads and BV geometry, MPIM preprint, 2006.

[3] S. Barannikov and M. Kontsevich. Frobenius manifolds and formality of Lie algebras of polyvector fields, Internat. Math. Res. Notices, 14 (1988), 201-215.

[4] J. Conant and K. Vogtmann. On a theorem of Kontsevich. Preprint math.QA/0208169.

[5] S. Eilenberg and J.C. Moore. Adjoint functors and triples. Illinois J. Math., 9 (1965), 381-389.

[6] W.L. Gan. Koszul duality for dioperads. Math. Res. Lett., 10 (2003), 109-124.

[7] E. Getzler and J.D.S. Jones. Operads, homotopy algebra, and iterated integrals for double loop spaces. Preprint hep-th/9403055.

[8] E. Getzler and M.M. Kapranov. Modular operads. Compositio Math., 110 (1998), 65-126.

[9] V. Ginzburg and M.M. Kapranov. Koszul duality for operads. Duke Math. J., 76 (1994), 203-272. 
[10] A. Hatcher. Algebraic Topology. Cambridge University Press, 2002.

[11] M. Kontsevich. Formal (non)commutative symplectic geometry. In: The Gel'fand mathematics seminars 1990-1992. Birkhäuser, 1993.

[12] M. Kontsevich and Y. Manin. Gromov-Witten classes, quantum cohomology, and enumerative geometry. Commun. Math. Phys., 164 (1994), 525-562.

[13] T. Leinster. Higher operads, higher categories, volume 298 of London Mathematical Society Lecture Note Series. Cambridge University Press, Cambridge, 2004.

[14] S. Mac Lane. Natural associativity and commutativity. Rice Univ. Stud., 49 (1963), 28-46.

[15] M. Markl. Operads and PROPs. Preprint math.AT/0601129, to appear in Handbook of Algebra.

[16] M. Markl. Cotangent cohomology of a category and deformations. J. Pure Appl. Algebra, 113 (1996), 195-218.

[17] M. Markl. Models for operads. Comm. Algebra, 24 (1996), 1471-1500.

[18] M. Markl. Simplex, Associahedron, and Cyclohedron. Contemporary Mathematics, vol. 227, 235-265, 1999.

[19] M. Markl, S. Shnider, and J. D. Stasheff. Operads in Algebra, Topology and Physics, volume 96 of Mathematical Surveys and Monographs. American Mathematical Society, Providence, Rhode Island, 2002.

[20] M. Markl and A.A. Voronov. PROPped up graph cohomology. Preprint math.QA/0307081.

[21] S.A. Merkulov, Strong homotopy algebras of a Kähler manifold, math.AG/9809172, Intern. Math. Res. Notices 3 (1999), 153-164.

[22] S.A. Merkulov.PROP profile of Poisson geometry. Arxiv math.DG/0401034 Commun. Math. Phys., 262 (2006), 117-135.

[23] S.A. Merkulov. Nijenhuis infinity and contractible dg manifolds. Arxiv math.AG/0403244, Compositio Mathematica, 141 (2005), 1238-1254.

[24] S.A. Merkulov. PROP profile of deformation quantization and graph complexes with loops and wheels. Preprint math.QA/0412257.

[25] A. Schwarz. Geometry of Batalin-Vilkovisky quantization. Commun. Math. Phys., 155 (1993), 249-260.

[26] J.D. Stasheff. On the homotopy associativity of H-spaces, I II. Trans. Amer. Math. Soc., 108 (1963), 272-292 \& 293-312.

[27] B. Vallette. A Koszul duality for props, math.AT/0411542, to appear in Trans. Am. Math. Soc.

[28] B. Vallette. Koszul duality for PROPs. Comptes Rendus Math., 338 (2004), 909-914.

M. Markl: Math. Inst. of the Academy, Žitná 25, 11567 Prague 1, The Czech Republic

E-mail address: markl@math.cas.cz

S. Merkulov: Department of Mathematics, Stockholm University, 10691 Stockholm, Sweden

E-mail address: sm@math.su.se

S. Shadrin: Department of Mathematics, Stockholm University, 10691 Stockholm, Sweden and Institute of System Research, Nakhimovskil prospekt 36-1, 117218 Moscow, Russia

E-mail address: shadrin@math.su.se, shadrin@mccme.ru 